\title{
The XVth World Congress of Psychiatric Genetics, October 7-11, 2007: Rapporteur summaries of oral presentations
}

Rapporteurs (in alphabetical order): Anna Alkelai (1), Amber Baum (2), Melanie Carless (3), James Crowley (4), Tania DasBanerjee (5), Emma Dempster (6), Sophia Docherty (7), Elizabeth Hare (8), Michael J Galsworthy (9), Deepak Grover (10), Dylan Glubb (11), Robert Karlsson (12), Jonathan Mill (13), Srijan Sen (14), Marlon P. Quinones (15), Eric J. Vallender (16), Ranjana Verma (17), Neetha.N.Vijayan (18), Sandra Villafuerte (19), Aristotle N. Voineskos (20), Heather Volk (21), Lan Yu (22), Petra Zimmermann (23), Coordinated by: Lynn E. DeLisi**

(1) Biological Psychiatry Laboratory, Dept. of Psychiatry, Hadassah - Hebrew University Medical Center, Jerusalem, Israel, E-mail: alkelai. ana@gmail.com

(2) Genetic Basis of Mood and Anxiety Disorders Unit, National Institute of Mental Health, NIH, Bethesda, MD 20892 USA. E-mail: bauma@mail.nih.gov

(3) Southwest Foundation for Biomedical Research, Department of Genetics, 7620 NW Loop 410 San Antonio, TX, 78227-5301, Ph: 210258 9766, Fax: 210258 9444, E-mail: mcarless@sfbrgenetics.org

(4) Department of Genetics, University of North Carolina-Chapel Hill, 4113 Neurosciences Research Building, 103 Mason Farm Road, Chapel Hill, NC 27599, Phone: (919) 966-9576, Fax: (919) 966-3630, E-mail: crowley@unc.edu

(5) 750 East Adams Street, SUNY Upstate Medical University, Dept. of Neuroscience and Physiology, Rm 3279, WHA, Syracuse, NY-13210, E-mail: tdas80@yahoo.com

(6) SGDP Centre, Institute of Psychiatry, De Crespigny Park, London, SE5 8AF, U.K, E-mail e.dempster@iop.kcl.ac.uk

(7) Social Genetic and Developmental Psychiatry Centre (PO82), Institute of Psychiatry, King's College London, De Crespigny Park, Denmark Hill, London, SE5 8AF UK, E-mail: Sophia.Docherty@iop.kcl.ac.uk

(8) South Texas Psychiatric Genetics Research Center, 454 Soledad Street, Suite 200, San Antonio, TX 78205, E-mail: hare@ uthscsa.edu

(9) Institute for Biomedical Informatics (IBMI), Faculty of Medicine, University of Ljubljana, Slovenia, E-mail: michael. galsworthy@mf.uni-lj.si

(10) Department of Psychiatry and Behavioral Sciences, Johns Hopkins School of Medicine, Baltimore, MD 21287, E-mail: dgrover6@jhmi.edu

(11) Gene, Structure and Function Lab,Department of Pathology, University of Otago, Christchurch, Christchurch Mail Centre, Christchurch 8140, New Zealand, E-mail: dylan.glubb@otago.ac.nz

(12) Department of neuroscience, Karolinska institutet, Retzius v 8, B2:4, 17177 Stockholm, Sweden, E-mail: robkar@gmail.com

(13) Institute of Psychiatry, De Crespigny Park, Denmark Hill, London SE5 8AF, E-mail: Jonathan.mill@iop.kcl.ac.uk

(14) Department of Psychiatry, 300 George Street \#901, Yale University,New Haven, CT 06511, E-mail: srijan.sen@yale.edu

(15) The University of North Carolina at Chapel Hill, Department of Psychiatry, Division of Biomarker Development, Center of Excellence for Research and Treatment of Bipolar Disorder (CERT-BD). 10616 Neuroscience Hospital, CB\#7160, Chapel Hill, NC 27599-7160, E-mail: quinones@med.unc.edu, Phone: 919-966-8832

(16) New England Primate Research Center, Harvard Medical School, One Pine Hill Drive Southborough, MA 01772, Phone: 508-624-8194, Fax: 508-786-3317, E-mail: eric_vallender@hms.harvard.edu

(17) Department of Psychiatry and Behavioral Sciences, Johns Hopkins School of Medicine, E-mail: verma2@jhem.jhmi.edu

(18) Human Molecular Genetics Lab, Rajiv Gandhi Centre For Biotechnology, Poojapura, Thycaud post, Trivandrum-695014, Kerala, India. Tel: +91 471 2341716, 2347975, Fax: +91 471-2348096, E-mail: neethav@yahoo.com

(19) Molecular \& Behavioral Neuroscience Institute, University of Michigan, 109 Zina Pitcher Pl. 5063 BSRB, Ann Arbor, Michigan 48109, E-mail: svillafu@umich.edu

(20) R31, 250 College St., Neurogenetics Section, Centre for Addiction and Mental Health, University of Toronto, E-mail: aristotle.voineskos @utoronto.ca

(21) Division of Biostatistics, Department of Preventive Medicine, University of Southern California 1501 San Pablo Street, ZNI 437, Los Angeles, CA 90089, Phone: (323)442-2489, Fax: (323)442-2448 E-mail: heather.volk@keck.usc.edu

(22) Institute for Nutritional Sciences, Shanghai Institute for Biological Sciences, CAS, Shanghai, China; Bio-X center, Shanghai Jiao Tong University, Shanghai,China, E-mail: biolan.yu@gmail.com

(23) Max Planck Institute of Psychiatry, Molecular Psychology Unit, Kraepelinstrasse 2, 80804 Munich, Germany, Phone: ++ 49-89-30622 237, Fax: ++ 49-89-30622 544, E-mail: pzimmer@mpipsykl.mpg.de

**address correspondence to: LE DeLisi (Chairperson of the Student Awardee program for the WCPG), 650 First avenue, 5th floor, Department of Psychiatry, New York University School of Medicine, NY, NY 10016, E-mail: DeLisi76@AOL.com

Acknowledgement: This program was supported by a grant from the USA National Institute of Mental Health (R13MH60596; LE DeLisi, PI) and a grant from the USA National Institute of Drug abuse and National Institute of Child Health and Human development (R13DAO22792; LE DeLisi, PI).

DOI 10.1002/ajmg.b.30711

(C) 2008 Wiley-Liss, Inc. 


\begin{abstract}
The World Congress of Psychiatric Genetics (WCPG) has become an annual event since the early 1990's sponsored by the International Society of Psychiatric Genetics (ISPG). Each year the latest published and unpublished findings are aired for discussion by representatives of the majority of research programs on this topic world-wide. The 2007 congress was held in New York City and attracted over 1000 researchers. The topics emphasized included results from whole genome association studies, the significance of copy number variation and the important contributions of epigenetic events to psychiatric disorders. There were over 20 oral sessions devoted to these and other topics of interest. Young investigator recipients of travel awards served as rapporteurs to summarize sessions and these summaries follow.
\end{abstract}




\section{Table of Contents:}

Introduction

1. Genome-wide association studies of bipolar disorder and schizophrenia

2. Genome-wide studies of autism and ADHD

3. Genome-Wide Association Studies of Depression, Panic Disorder and Antidepressant Treatment Response

4. Statistical methods for genome-wide association studies

5. Brain imaging in genetic association studies

6. Endophenotypes in schizophrenia and bipolar disorder

7. Genetics of mood disorders

8. Is there a genetic overlap among the psychoses?

9. New genetics approaches to studies of dementia

10. New perspectives on schizophrenia candidate genes $\quad 20$

11. Genetics of substance abuse 22

12. Functional characterization of genes involved in nicotine dependence 25

13. Genetic influence on adult neurogenesis 26

14. Psychiatric genetics and pharmacotherapy 28

15. New bioinformatics and computational methods 32

16. Copy number variation: implication for psychiatric disease 33

17. Gene expression studies $\quad 35$

18. Sex chromosome aneuploidies and the sex chromosomes in psychiatric disorders 37

19. Epigenetics $\quad 38$

20. Gene-environment interactions $\quad 40$

21. Animal Models $\quad 41$

22. From genes to drugs, a workshop $\quad 43$ 


\section{INTRODUCTION}

The following series of short reports summarizes the information presented and discussed during the oral sessions of the XVth World Congress of Psychiatric Genetics (WCPG) held at the Marriott Marquis Hotel in New York City October $7^{\text {th }}$ to $11^{\text {th }} 2007$. Often times, while there is a record of abstracts written several months before presentations, there is no written recording of what has happened during the sessions for those meeting attendees who were participating in other overlapping sessions or for those individuals who were unable to attend the conference and are interested in this field. Thus, at the 2007 WCPG a new rapporteur program was established in order to provide a recording for the written record of what transpired during these interesting and intense days. Rapporteurs were selected from the group of student travel awardees who volunteered to take on this task as one of the ways they would participate in the congress. All reports are the impressions and interpretations of the Rapporteurs and thus the investigators they quote are not responsible for the accuracy of these reports. We hope to continue publishing summaries and impressions of young investigators who attend the WCPG each year and thank those who donated their time to this important effort to summarize the 2007 WCPG.

This year the spotlight seemed to be on results beginning to emerge from very large multicenter collaborations performing genome-wide association in multiple psychiatric disorders, ADHD, autism, schizophrenia and bipolar disorder. In addition there was a new awareness of the significance of copy number variation throughout the genome as well as epigenetic events and the creation of an "epigenome" that may be more crucial in understanding the genetics of brain disorders than simply the variation in DNA sequence. The field has without question made considerable progress since the first gatherings of the newly formed field of psychiatric genetics in the 1980's; but clearly it still has a long way to go before the mechanisms that contribute to the development of these uniquely human disorders of thinking and communication that are at the heart of this field are uncovered. 
1. GENOME-WIDE ASSOCIATION IN BIPOLAR DISORDER AND SCHIZOPHRENIA

\section{Reported by Marlon Quinones, Amber Baum,} and Lynn E DeLisi

Whole genome-wide association studies (GWAs) were certainly the highlight of this congress. In previous years relatively small samples of families were used to try and define patterns of chromosomal linkage that would indicate regions for major genes for schizophrenia and other psychiatric disorders. These studies largely failed to produce consistent findings and were followed by a variety of candidate gene association studies. However, it was recently felt that the first generation of studies failed because, not only were the sample sizes much too small, but the number of markers used to survey the genome were clearly not sufficient. Thus with the development of new chip technology, SNP maps of the genome now numbering almost a million, and multicenter collaborations a reality, several new GWAs (Genome-Wide Association studies) are now being reported. The data presented at this 2007 meeting were just the beginning.

For bipolar disorder, the congress opened with a plenary session summarizing four major bipolar GWAs with data analyzed, with others to be reported in the near future. Nick Craddock (Cardiff University, Wales) presented results from the Wellcome Trust Case-Control Consortium (WTCCC) study, which was recently published in Nature as part of a larger association study that included six other complex disorders (The Welcome Trust Case Consortium, 2007). Using 2000 bipolar cases and $3000 \mathrm{UK}$ controls genotyped on the Affymetrix 500K chip, the WTCCC found SNP associations of small effect sizes in novel regions and near plausible gene candidates such as a GABA receptor (GABRB1), a glutamate receptor (GRM7), and the presynaptic protein synapsin III (SYN3), although the strongest result, which also had an odds ratio over 2 , was in a novel region on chromosome 16. Craddock also presented a new metaanalysis of the WTCCC data and the study from Baum et al. (see below) which showed that the set of SNPs reported as replicated in the Baum et al. study had more significant $\mathrm{p}$-values in the WTCCC sample than would be expected by chance.

Pamela Sklar (Massachusetts General Hospital and the Broad Institute, MIT) reported on a study combining the Systematic Treatment Enhancement Program for Bipolar Disorder (STEP-BD) sample with a UK sample (collected by Hugh Gurling's group at The University College, London) which totaled 1461 bipolar and 2008 unaffected controls genotyped on the Affymetrix 500K chip. Gene candidates identified in this study included Myosin 5B (the protein encoded by this gene is expressed in dendritic spines) and EGFR. When comparing this sample's finding with the WTCCC's and Baum et al's findings, SNPs in the gene DFNB31 (Whirlin) were found to be associated in all three samples, although the opposite allele was associated in the WTCCC. Notably,
Dr. Sklar's combined analysis plus an additional extension sample of about 1000 individuals also revealed association to a SNP in the calcium channel, voltagedependent, L type, alpha 1C subunit (CACNA1C), a voltage-dependent calcium channel subunit, which had a small OR (1.19) but a robust $P$-value.

Amber Baum (MAP Genetics, NIMH Intramural Program) reported on a study that used a narrowlydefined subset of the NIMH Bipolar Disorder Consortium sample and a larger sample collected from Germany (see below). The initial results, using a DNA pooling strategy with the Illumina HumanHap550 chip to genotype a total of 1233 cases and 1439 controls, were published in May (Baum et al., 2007) and implicated 80 genes. Baum presented new results from a randomeffects meta-analysis based on the Baum et al. and WTCCC studies. Evidence of association in all three samples was shown for two SNPs near the genes JAM3 and ZIP3. No support was found in the WTCCC sample for the most significant SNP reported in Baum et al., which resides near the lithium-related gene diacylglycerol kinase eta (DGKH).

Laura Scott (Pritzker Consortium, University of Michigan) also presented results from the NIMH Bipolar Disorder Consortium sample. The Pritzker Consortium genotyped about 1,000 NIMH cases on the Human Hap550, along with a similar number of controls. No genome-wide significant findings emerged, but support was seen for DGKH and a variety of novel loci. Sven Cichon (University of Bonn, Germany) had just completed individual genotyping on the HumanHap550 of 772 cases and 876 controls, one of the samples that was pooled in the Baum et al. study. Preliminary results supported key findings in the published paper but analyses were ongoing at the time of the meeting.

Looking beyond specific candidates, these 5 GWAS in BD tell us about where the field is and where it is heading. There was a unanimous agreement that collaboration between groups was now more than ever indispensable to reach the number of subjects required to identify the likely multiple genes of modest effect sizes that maybe accounting for the well defined genetic susceptibility to BD. Also, it was shown that i) metaanalytical tools provide an excellent opportunity to harvest results from smaller studies and lead to identifying candidate genes more likely to be replicated in future studies; ii) studies based on initial pooling of samples may be a viable alternative to reduce costs during initial screening phases; and iii) we may be missing several SNPs due to the losses during the QC. For instance, in the study presented by Dr. Sklar, 500,568 SNPs were genotyped using the Affymetrix GeneChip Human Mapping Array. For analysis only SNPs met a variety of stringent criteria resulting in a final dataset of 372,193 SNPs. The overall genotyping call rate was $99.4 \%$. This limited coverage may help explain why the previously closely studied candidate gene studies have not shown up in GWAs. Nevertheless, with bipolar 
genome-wide association studies, some genes seemed to standout across independent samples and these will likely receive considerable attention in follow-up studies, while other gene associations were found in one sample and not another. Whether the latter are spurious findings will be clarified with more future studies.

In another session, five major GWA studies on schizophrenia were represented, one put together under the leadership of Pamela Sklar at the Broad Institute, another from the Wellcome Trust collaboration in the UK and presented by Michael O'Donovan, another on a national German sample, presented by Sven Cichon from Bonn, the fourth from Shanghai led by Lin He and a USA sample from the subjects who participated in the CATIE neuroleptic comparison trial. All studies used either a $500 \mathrm{~K}$ or greater chip from Affymetrix or Illumina. The Broad Institute sample consisted of 3800 cases of schizophrenia and 4200 controls, the Wellcome Trust sample 1600 cases and 3700 controls, while the German, Shanghai and CATIE samples were smaller with 493 cases and 1356 controls (German), 200 cases and 200 controls (Shanghai) and 741 cases and 751 controls (CATIE). While the CATIE sample failed to show any areas of genome-wide significance, the other 4 had several regions of interest. What was most notable was that unlike what emerged in independent bipolar GWAs thus far, with schizophrenia there was no consistent pattern of significance across studies and none of these studies implicated as candidates any of the previously thought of candidate genes, such as neuregulin, dysbindin, COMT, BDNF or G72. However, genome-wide association studies are still in their infancy and their outcome and usefulness are yet to be determined. Many of the studies will need to be combined to yield larger N's with the knowledge that diagnosis and clinical information may vary from center to center producing some level of noise within the data. While the N's may need to be substantially higher for consistent results to emerge, the alternative view that the epigenome and environmental influence must be accounted for will need to be aired. The field currently awaits reports from the NIH Foundation-Pfizer GAIN program that has as one of its projects genome-wide association of schizophrenia using over 3000 Caucasian USA cases and 3000 controls.

\section{$\underline{\text { Reference }}$}

The WellcomeTrust Case Control Consortium. Genomewide association study of 14,000 cases of seven common diseases and 3,000 shared controls. Nature 2007; 447: 661-648.

\section{GENOME-WIDE STUDIES OF AUTISM AND ADHD Reported by Sophia Docherty}

Though the central phenotypes were autism and ADHD, the aspect which most notably united this group of oral presentations, as well as a number of the conference's other talks, was the attention devoted to the technical and analytical obstacles encountered when conducting research on a genome-wide scale. Whilst the study designs discussed were impressive and will no doubt yield findings of great import for their fields, with many of the results presented being far from final, the suggested methodological approaches - readily applicable across most phenotypes - were just as captivating as the substantive content.

Mark Daly was the first to present his 'Genome-wide association study in autism'. His group was instrumental in the development of the Genome-Wide Human SNP Array 5.0 (Affymetrix), to combine both SNP and CNV genome-wide coverage in a single assay. Rather than simply detecting CNVs, the 5.0 - and now the million SNP chip 6.0 - also offers quantitative information in order to generate a CNV genotype. Using this new platform, Daly studied 751 families, each comprising two or more effected siblings, drawn from the Autism Genetic Resource Exchange (AGRE). A CNV was detected along an identical $600 \mathrm{~Kb}$ stretch of $16 \mathrm{p}$ in 5 patients, but was not seen in their families. A CNV at this position was also detected in a further 8 patients in 3 families, where it was shown to segregate with autism. In a separate screen of 512 clinically referred children with developmental delay ASD, a deletion at this stretch was detected in 5 children, a duplication in 4 more. No deletion or duplication was found in any of the 450 controls used.

Daly's finding of a CNV present in 1-2\% of the autism cases studied seems consistent with the recent literature suggesting a role for de novo CNVs in autism ${ }^{\mathrm{i}}$. However, this was the only highly recurrent de novo CNV found, and upon discussion with the panel we were reminded that until the base rate of de novo $\mathrm{CNV}$ mutations throughout the normal population has been gaged via analysis of several generations of control populations, causal relationships can not be concluded. Preliminary SNP analyses were also presented, with 51 SNP associations at $\mathrm{p}<10^{-4}, 6$ at $\mathrm{p}<10^{-5}$, a robust association in chromosome 1 and promising findings in the DAOA gene mentioned. However, despite the completion of basic quality control measures in PLINK and Birdseed (2.0) which reduced the number of SNPs in the study from 470,000 to 400,000, a good deal more data cleaning is required before Daly would be ready to present his final results.

The importance of data cleaning was emphasized to an even greater extent in Benjamin Neale's presentation of the 'Quantitative analysis of ADHD from the IMAGE genome-wide association study'. After using Perlagen arrays to genotype trios, 27 individuals were excluded from the study on the basis of low call rates $(<0.87)$, gender discrepancies, sample heterozygosity and Mendelian inconsistencies, with 1865 parents and 936 children remaining. SNP exclusion was dependent upon allele frequency - with more stringent criteria applied 
to those of low MAF - and eliminated 144,511 ( 25\%) SNPs from further analyses. Both TDT analysis in PLINK, and comparison of $\mathrm{X}^{2}$ for high and low $\mathrm{p}$-value SNPs, revealed a systematic bias in rare and common allele overtransmission between those SNPs of high and low $(<0.3)$ MAFs. Strict Lambda correction was applied to compensate for the error present in the higher MAF SNPs - which Neale postulated may have remained after the less stringent initial quality control. Neale expects few detectable effects in his data, and is therefore of the strong opinion that, although a degree of power may be lost, strict quality control is wise. Just as in Daly's first-pass results, no SNPs reached genomewide significance, however Neale views this as simply 'a state of mind', with replication proving the true test for associations.

In an attempt to identify true associations within the mass of data generated, Neale's group then supplemented their p-values with functional landmark information and re-ranked their SNPs based on these biologically weighted scores. Replication studies involving thousands of subjects are already planned - however it is becoming apparent that far larger samples will be needed in the future - and Neale seemed adamant that aggressive data sharing and collaboration were the only answers. Indeed, the data from all of the studies discussed in this session - and many others at the conference as a whole - will be made publicly available. Neale also intends to incorporate haplotype information into this investigation, using HapMap-based imputed genotypes, which should go some way to increasing its power.

In her 'CHOP ADHD cohort GWA study', Josephine Elia is currently in the process of genotyping 500 trios on the Illumina HumanHap 550 beadchip platform. Again, no SNPs have reached genome-wide significance, however Elia's group have focused upon SNPs found inside or within $5 \mathrm{~Kb}$ up- or downstream of previously reported ADHD candidate genes. So far, several of these SNPs have been suggestive of association - including DAT 1 and SNAP-25-however only the association of $A D R A 1 A$ remained significant at $\mathrm{p}=0.05$ with no apparent parent of origin effects. The treatment of candidate genes was the focus of some discussion, with Stephen J Glatt proposing that in GWA research perhaps one might be more lenient with the p-values of previously identified candidate genes considering the prior evidence we have for association, however Daly felt that there are very few candidate genes worthy of such leniency. Neale was of the opinion that a blind look at the genome is the best approach, but added that metaanalyses incorporating all of the data generated on a phenotype should continue. Genotyping of Elia's sample is still underway and she hopes to recruit more subjects. The group has also planned longitudinal analyses to assess genotypic effect on the phenotypic patterns her group has observed, which suggest that ADHD contains various clusters of phenotype, which vary with age.
Maja Bucan discussed 'Novel computational biology approaches for the analysis of high-density SNP genotype data'. With an initial focus upon endophenotypes within the mouse, Bucan's group identified potential candidate genes. They then employed a bioinformatics approach to compile a list of 3000 genes acting in the synapse and pre-synaptic neuron. The group also searched for extremely large conserved noncoding elements across different species. Examination of the regions has begun, and Bucan called for more model organism work and better annotation of the genome. The next step would be to concentrate on those SNPs and CNVs falling within and near these candidate genes and conserved elements during the group's analysis of genome-wide association data from the entire AGRE sample, however, only 188 families have been genotyped on the Illumina Human HapMap 550 beadchips so far. Again, genome-wide significance has not been reached, although this is to be expected with the sample size as it stands.

The group has also developed and tested a novel CNV detection algorithm - PennCV. PennCV outputs a comprehensive report for each gene - as opposed to simply for each individual - so that one can assess the sizes, frequency and parent of origin affects of $\mathrm{CNV}$ s within any one gene. In the 2500 individuals the group assessed, 61,000 CNVs were found, with an extremely uneven spread throughout the subjects. 846 common ( $>1 \%$ of the sample) CNVs were identified, and 336 de novo CNVs were detected in children with autism, some of which fell within previously reported candidate genes. Again, the final results were not yet available. However Bucan's talk, along with the other three in this session, does hold promise for the identification of some of the SNPs and CNVs influencing Autism and ADHD. Moreover, the methods developed and discussed by the speakers here, from genotyping platforms to novel research approaches to analytical algorithms, may be used to enhance the study of many complex phenotypes.

\section{Reference}

Sebat J, Lakshmi B, Malhotra D, Troge J, Lese-Martin C, Walsh T, Yamrom B, Yoon S, Krasnitz A, Kendall J, Leotta A, Pai D, Zhang R, Lee YH, Hicks J, Spence SJ, Lee AT, Puura K, Lehtimaki T, Ledbetter D, Gregersen PK, Bregman J, Sutcliffe JS, Jobanputra V, Chung W, Warburton D, King MC, Skuse D, Geschwind DH, Gilliam TC, Ye K \& Wigler M. 2007. Strong association of de novo copy number mutations with autism. Science 316:445-449.

\section{GENOME-WIDE ASSOCIATION STUDIES OF DEPRESSION, PANIC DISORDER AND ANTIDEPRESSANT TREATMENT RESPONSE Reported by Melanie Carless}

Depression is symptomatically characterized by alterations in cognitive, psychomotor and emotional processes 
(Lesch, 2004). The clinical features, severity and course of the illness are highly heterogenous within patients and the genetic basis of depression is highly complex, polygenic and epistatic (Lesch, 2004). Anxiety disorders, including panic disorder, display common symptoms with depression with approximately $60 \%$ of depressed patients also experiencing anxiety symptoms (Baldwin et al., 2002). Pharmacological agents used for depression include enzyme inhibitors, uptake blockers and receptor blockers; with selective serotonin reuptake inhibitors (SSRIs) being the most widely prescribed drug for mood and anxiety disorders (Artigas et al., 2002; Golden, 2003). The complexity of mood and anxiety disorders has made the identification of susceptibility genes difficult. In the past, candidate gene analyses and linkage studies have had limited success in consistently identifying susceptibility genes contributing to for example, major depression. The current classification scheme, which is based on clusters of symptoms rather than describing a homogenous disorder, is likely to contribute to this (Hasler et al., 2004). Genome-wide association studies (GWAS) have been successfully employed to identify genetic regions associated with a number of diseases (WTCCC, 2007) and may also provide a sound bench mark for the delineation of regions involved in depression and panic disorder.

The GWAS of mood and anxiety disorder session opened with presentations by Drs Patrick Sullivan and Steven Hamilton examining GWAS of major depressive disorder (MDD). Dr Sullivan is currently performing a large scale GWAS using 1,860 patients with MDD in the Netherlands Study of Depression and Anxiety (NESDA) cohort and an equal number of controls obtained from the Netherlands Twin Registry (NTR). His aim was to perform a two-stage study, initially to examine 430,000 SNPs using a Perlegen single nucleotide polymorphism (SNP) array and to then replicate the most promising 15,000 SNPs, further refining this to a second replication of 20 SNPs. Although no results were presented, these studies are underway and the identification of genetic variations associated with MDD susceptibility are promising.

Dr Hamilton presented preliminary data on the Sequenced Treatment Alternatives to Relieve Depression (STAR*D) cohort consisting of a diverse collection of white non-Hispanics $(\mathrm{n}=1,279)$, African Americans $(\mathrm{n}=313)$ and white Hispanics $(\mathrm{n}=247)$ individuals across the United States. For this study, a subset of 633 white non-Hispanic cases from the STAR*D cohort were collected through a clinical trial examining drug response and were genotyped using the Affymetrix $500 \mathrm{~K}$ mapping array and 5.0 array platforms. Also, 879 independent controls from the National Institute of Mental Health (NIMH) had been previously genotyped using an Affymetrix platform for this study. In this preliminary analysis, seven SNPs were identified as possibly being associated. However, as there were no other SNPs in the surrounding areas that were significant, it was suggested that these may be false positive results. Dr Hamilton commented on the fact that findings need to be replicated, both in a larger STAR*D cohort and also in an independent population.

The next two presentations, delivered by Dr Brian DeChairo and Mr Jeffrey Kraft, pertained to patient response data following the administration of SSRIs. Dr DeChairo presented a meta-analysis of eight different pharmacological response studies investigating the administration of Sertraline $(n=1)$, Fluoxetine $(n=2)$ and Paroxetine $(n=5)$. Across the eight studies 477 individuals were administered a SSRI and 451 individuals were administered a placebo and all samples were genotyped using the Perlegen SNP array platform. Dr DeChairos' approach was to analyze responders administered an SSRIs (endpoint) and to compare this to responders administered a placebo to determine the "placebo effect". After replication in two populations two SNPs, rs1324058 and rs17670808, were significantly associated with SSRI response. Dr DeChairo further concluded that data from smaller populations should be merged prior to analysis as significant results obtained from small populations are unlikely to replicate in another population due to their lack of statistical power in both studies.

Mr Kraft investigated both response and remittance (as endpoints) in the STAR*D cohort and found a number of genetic variants to be significantly associated with each of the SSRI endpoints, many of which overlapped ( $>50 \%$ of response associated SNPs were also remittance associated SNPs). Mr Kraft investigated 1,279 individuals (53\% non-responders, $14 \%$ responders, 33\% remitters) using the Affymetrix 500K mapping array and 5.0 array platforms. Using a statistical significance threshold of $\mathrm{p}<1 \times 10^{-4}$, five SNP clusters (1p34.3, 8q11.23, 9p21.1-21.2, 19q13.12, 21q21.3) were associated with the remittance phenotype. The $1 \mathrm{p} 34.3$ and 9p21.1-21.2 SNP clusters were also associated with the responder phenotype, as were some other individual SNPs.

The final presentation of the session, delivered by Dr Angelika Erhardt, outlined a preliminary GWAS of anxiety disorders (80\% panic disorder) using the Illumina 300K BeadChip. Employing a two stage study design, this German study genotyped 216 cases and 222 controls in stage one and an independent ascertainment of 236 cases and 241 controls in stage two. Of the five genes (KPNA4, FHIT, CRIM1, PLCB1 and TMEM132D) exhibiting suggestive significance across both study stages, Dr Erhardt reported the hypothetical protein TMEM $132 D$ to satisfy genome-wide correction factors in both populations. Although results were preliminary, the analysis is promising and further studies are underway to investigate expression differences in animal models of pathological anxiety.

One of the major points to arise during this session and subsequent discussion was the need for a collaborative effort. In particular, Dr Sullivan commented on the 
formation of a Psychiatric GWAS Consortium originating in May 2007, and how it is now developing into a powerful tool for the detection of genetic variation associated with various psychiatric disorders. Currently funded by NARSAD, the consortium aims to draw upon approximately 30 research cohorts with a total of approximately 50,000 individuals $(31,000$ cases and 19,000 controls). Of the five different disorders to be studied (attention deficit-hyperactivity disorder, autism, bipolar disorder, major depression and schizophrenia), major depression cases will contribute largely to the study with about 8,000 cases and 8,000 controls. It is encouraging to note that all research groups that have been approached about the consortium have had a positive reaction, indicating a shared ideology for collaborations to increase the power of GWAS.

One of the major concerns raised during this GWAS session was the extent of heterogeneity across studies, an issue that is likely to become much more prominent with the development of a consortium. The discussion moderator, Dr Michael Gill, commented that phenotypic definition needs to be consistent across studies with a subsequent response from Dr Hamilton that each study does employ a subjective means of diagnosis and selection; hence it would be difficult to eliminate heterogeneity. This opinion was echoed throughout the discussion by each of the speakers with the consensus that population heterogeneity does exist and that it may cause problems in studies of this magnitude. Dr Gill questioned whether this problem can be fixed, however no formal solution was voiced during this session. Diagnostic heterogeneity is therefore an important issue to consider during study design, analysis and result interpretation as the advent of false negative results and an inability to replicate results is highly likely.

Phenotypic classification is not only a heterogeneity issue but it can also shroud the extent of information obtained from a study. The possibility of a "pheno-wide" study was opened for discussion, specifically whether phenotypic variants could also be associated with specific genetic variants. Dr DeChairo commented that we could look for association by narrowing the phenotype, but that we could also broaden the phenotype. $\mathrm{Dr}$ DeChairo also questioned the subjectivity of diagnosing psychiatric disorders and whether single or multiple phenotypes will be consistently diagnosed. The question of whether drug tolerability can be considered as a phenotype was also posed. Dr Hamilton responded by suggesting that we may be able to identify markers that are predictive of a clinical endpoint as well as those that define genes and genetic pathways associated with psychiatric diseases.

Another important issue that was raised was a lack of stage two design and the question of "where we go from here" remains. In response, replication studies were proposed to confirm the "top hits" (i.e. the top 2,500$50,000 \mathrm{SNPs}$ ). However, this response was debated in that it would be financially ineffective to examine 50,000
SNPs as part of a replication and/or second stage of a study. Meta-analyses were also suggested to potentially provide more insightful information and that perhaps the best way to determine if a particular genetic variant is significantly associated with any psychiatric disorder is to combine genotypic information from many studies. Dr Gill also raised a concern that if we fail to find something in these smaller populations, this may affect our ability to get funding for larger studies.

To date, meta-analyses reported from The Wellcome Trust Case Control Consortium (WTCCC) have identified a number of novel genetic regions associated with various non-psychiatric disorders such as coronary artery disease (9p21.3), Crohn's disease (3p21, 5q33, 10q24, 18p11), rheumatoid arthritis (7q32, in females only), type 1 diabetes (12q13,12q24,16p13) and type 2 diabetes (6p22, 16q12) (2007). A number of candidate genes reside in these genetic regions, although these have not been functionally verified in the diseases. However, this proof of concept gives promise to the future of the Psychiatric GWAS Consortium and the identification of genes associated with psychiatric illness, including major depression and panic disorder. We may still have a long way to go in the identification of genes and genetic pathways involved in psychiatric diseases but the data presented during this symposium, along with the discussion and future collaborative efforts generated promises to advance our knowledge of the genetics underpinning psychiatric disorders.

\section{References}

Artigas F, Nutt DJ, Shelton R. 2002. Mechanism of action of antidepressants. Psychopharmacol Bull 36(Suppl 2):123-132.

Baldwin DS, Evans DL, Hirschfeld RM, Kasper S. 2002. Can we distinguish anxiety from depression? Psychopharmacol Bull 36(Suppl 2): 158-165.

Golden RN. 2003. Efficacy and tolerability of controlledrelease paroxetine. Psychopharmacol Bull 37(Suppl 1):176-186.

Hasler G, Drevets WC, Manji HK, Charney DS. 2004. Discovering endophenotypes for major depression. Neuropsychopharmacology 29(10):1765-1718.

Lesch KP. 2004. Gene-environment interaction and the genetics of depression. J Psychiatry Neurosci 29(3):174184.

The Wellcome Trust Case Control Consortium. 2007. Genome-wide association study of 14,000 cases of seven common diseases and 3,000 shared controls. Nature $p$ 661-678.

\section{STATISTICAL METHODS FOR GENOME-WIDE ASSOCIATION STUDIES Reported by Robert Karlsson}

Four presentations describing new statistical methods related to genome-wide association studies were given 
by Goncalo Abecasis, Shaun Purcell, Itsik Pe'er, and Frank Dudbridge.

Goncalo Abecasis: Turning a flood of data into a deluge: "in silico"genotyping for genome-wide association scans. Dr. Abecasis discussed the rationale for 'in silico genotyping, and methods to perform it. His message was that we can produce quite dense SNP genotypes today, but it would be even better to have even denser genotypes, in order to pinpoint disease loci more precisely. Furthermore, for maximum power in GWAS, we would like to have as large sample sizes as possible. Manufacturing denser SNP chips and collecting more individuals is very expensive, but we can get further information from the data we have today by imputing genotypes with in silico methods.

Two approaches were suggested: Increase genotype density in related individuals, where most are genotyped only on a few markers, by following the segregation of these markers through the pedigree to determine where recombinations happen, and then filling in the missing markers from chromosome segments shared with more densely genotyped relatives. Alternatively increase genotype density in unrelated (actually distantly related due to population history) individuals by the same principle, but using e.g. Hapmap data (which is genotyped at about 2.5 million SNPs) to 'fill in the blanks'. A maximum-likelihood estimate was used for each genotype imputation. This method has been tested on various datasets, and found to be more powerful than analyzing the data using other approaches. The algorithm can also use the combined Hapmap data (all groups) to make decent genotype imputations.

Among the several questions posed, Dr. Abecasis was asked whether this new software will be available on his website, and if guides and tutorials for performing the analysis will be provided, as has been made for his other software packages. He replied that this will happen soon, but did not give an exact time frame. Another question was on how long the analysis takes to perform. Abecasis' reply was that currently on an ordinary PC the imputation takes about one day per chromosome. Another audience member asked how robust this method is to genotyping errors. To this Dr. Abecasis replied that, as always, if you don't do a decent quality control on your data, you cannot trust your results. With that in mind, the results after imputing genotypes do not get any worse than before imputation on data with genotyping errors. The last question for Dr. Abecasis was whether his method really is different from a (much simpler to compute) multiple regression based on known genotypes and their correlation with unknown genotypes. His reply to this was that it is different, because the MLE approach takes into account the local (non-linear) LD block structure of the genome, which is not processed accurately by a multiple regression.
Shaun Purcell: An examination of rare variation and extended segmental sharing in whole genome studies of bipolar disorder and schizophrenia. Dr. Purcell presented his progress in the development of a method for finding relatively recent and rare population-specific mutations associated with bipolar disorder and schizophrenia. His reasoning motivating this was that recent mutations that have spread in a population should come with a long haplotype region of linkage disequilibrium surrounding it, since it has not yet had time to break down from recombinations. This uncommonly long stretch of markers is shared among individuals with the disease caused by the mutation, and thus an association between haplotype and disease that is detectable by Purcell's algorithm emerges. Importantly, applied to the same whole genome SNP datasets currently being collected and analyzed with common variation in mind, this method aims to provide a complementary way to scan for rarer but more penetrant variants that would otherwise be missed. Dr. Purcell suggested an algorithm based on a Hidden Markov Model to detect long haplotypes that are shared both between chromosomes in the same individual (extended stretches of homozygosity) and between distantly related individuals. The latter method can be seen as a sort of population-based linkage analysis. Restrictions on the length of haplotypes are set to limit the analysis to fairly recent mutations, which approximately corresponds to haplotypes of over $1 \mathrm{Mb}$ of length. An important caveat when using this method is that different rates of relatedness in case and control groups due to sampling bias can have a huge influence on the outcome. Therefore extra care should be taken to avoid such bias.

The algorithm is implemented in Purcell's PLINK software package (Purcell et al., 2007), which is freely available on his website http://pngu.mgh.harvard.edu/ $\sim$ purcell/plink/. Dr. Purcell has tested his method on two real world datasets: genotypes from the bipolar patients in the STEP-BD study, and a subset of the WGAS genotypes from the International Schizophrenia Consortium. Results are still being interpreted and validated, but a few regions turned up as potentially interesting for haplotype sharing.

Itsik Pe'er: Genetic analysis of GWA data in an admixed population. Dr. Pe'er's presentation addressed the problem of admixed populations in whole genome association studies. WGAS are known to have the highest power if the sampled population has a homogeneous ancestry, but most often that is not the case. The problem with samples from admixed populations is that if the admixture is not taken into account during association analyses, p-values in an association study can be greatly inflated due to this admixture, rather than true disease association. Pe'er's software (Xplorigin) addresses this problem. Xplorigin is available (with $\mathrm{C}++$ source code) from http://www.cs.columbia.edu/ 
$\sim$ itsik/Xplorigin/Xplorigin.htm. Pe'er demonstrated his methods to be superior to the popular "Genomic Control" approach. On simulated data without a disease effect he compared them by quantile-quantile plots to an uncorrected association study (proportion of low p-values highly inflated), Genomic Control (better, but overcorrection leads to a deficiency of low p-values), and Principal Component Analysis (better still, but a small tendency towards overcorrection). Pe'er's methods called Ancestry Proportions (the most accurate-looking plot) and "Local ancestry" (similar to the PCA and Ancestry Proportions plots, but with a small tendency towards too low p-values) looked the most promising. The power of local ancestry and ancestry proportions analyses was also demonstrated in a simulation where a disease effect was present, and the Xplorigin treated data gave the clearest signal from the disease locus (again compared to Genomic Control and PCA).

One question posed was whether Pe'er had any suggestion on how to handle population-based samples from the USA, where 10-15\% of the population has African American ancestry. Pe'er's answer to this was that it is important to never throw away data, but we have to be very careful when we analyze it. We might not be able to perform the 'correct' analysis today, but development is ongoing in the area, so we should keep our data in anticipation of better analysis methods.

Frank Dudbridge: Estimating significance thresholds for genome-wide association scans. Dr. Dudbridge gave a presentation on how to determine the p-value threshold for significance in genome-wide association studies, something which is far from trivial due to among other things linkage disequilibrium between nearby markers on SNP chips, which makes Bonferroni correction inappropriate, and the fact that we don't know how many 'true positive' findings to expect in a GWAS.

Using permutations of data from the WTCCC blood donor control group, where half of the group randomly was assigned a 'case' status, Dudbridge could find a good approximation of the null distribution of minimum pvalues. This was repeated for subsets of SNPs with varying density, and for each density the $5 \%$ quantile of minimum p-values was calculated.

Extrapolating this to an infinitely dense marker map by fitting the monod curve to the effective number of tests, Dudbridge found a p-value threshold of $6.0 \times 10^{-8}$ to be appropriate for genome-wide significance. Since this threshold assumes an infinitely dense marker map, it should apply regardless of which panel a specific study uses. Dudbridge's suggested threshold is an order of magnitude smaller than that used in the WTCCC studies. However, of the 11 reported significant results from WTCCC:s studies, only 1 had a p-value in the critical interval $\left(6 \times 10^{-8}, 5 \times 10^{-7}\right)$, so using the threshold suggested by Dudbridge would not have made a big difference for the overall outcome of that specific study.
Reference

Purcell S, Neale B, Todd-Brown K, Thomas L, Ferreira MA, Bender D, Maller J, Sklar P, de Bakker PI, Daly MJ, Sham PC. 2007. PLINK Genetic Analysis Package. Am J Hum Genet 81:559-575.

\section{BRAIN IMAGING IN GENETIC ASSOCIATION STUDIES Reported by Aristotle N. Voineskos}

Brain imaging and genetics are two powerful lines of neuroscientific enquiry that when combined may be able to provide better answers than either technology alone. Though a statistical relationship between a risk allele and a clinical diagnosis may not be shown, there may be a significant effect of that allele on a biological process for the illness that can be demonstrated using brain imaging. However, the issues of multiple testing, small sample size, and spurious associations faced in both genetics and imaging can prove challenging in study design and analysis.

This report highlights various approaches and limitations of combining imaging and genetics by summarizing five presentations and the subsequent discussion in the symposium.

Presentation 1: Genome Wide Association Study of PET Scan Phenotypes (Dr. Xinmin Liu, NIH). This first study examined potential positron emission tomography (PET) imaging endophenotypes in mood disorders. These were defined as binding potential (BP) of [11C] DASB PET, a ligand for the serotonin transporter and [18F] TZTTP, a muscarinic receptor ligand. This is the first genome-wide association study examining positron emission tomography (PET) imaging endophenotypes in mood disorders. These phenotypes were defined as binding potential (BP) of [11C] DASB, a ligand for the serotonin transporter and [18F] TZTTP, a muscarinic receptor ligand in the PET. 144 unmedicated individuals underwent scanning with one or both of the above ligands. DNA samples were genotyped with 109,365 single nucleotide polymorphisms (SNPs) by use of the Illumina Infinium I chip. Genetic association testing with BP at either receptor was carried out by logistic regression controlled for ethnicity. Correction for multiple testing was made using the false discovery rate (FDR). For residual bias detection, a QQ plot was constructed and no residual bias was detected.

For the muscarinic receptor binding phenotype, the rs1519781 SNP was associated with [18F] TZTTP BP in a conserved region on chromosome $2 \mathrm{q} 23.3(\mathrm{p}=6.92 \times$ $\left.10^{-8}\right)$. The SNP rs2279508 near the SLIT1 gene was associated at $p=1.68 \times 10^{-6}$. SLIT1 encodes a protein thought to be involved in axonal migration. The implicated genes may play a role in mood disorders. 
Presentation 2: False Discovery in Psychiatric Imaging Genetics (Dr. Daniel Weinberger, NIH). This study was designed to determine the false positive rate in imaging genetics using genotypes (real and permuted) with low prior probability of association. Seven hundred twenty common nonconservative SNPs with $>10 \%$ minor allele frequency (MAF) that did not show significant association with schizophrenia in either family or case control datasets were chosen. Four hundred ninety two of these SNPs did not show association with cognitive measures and these were analyzed separately. For each SNP the effects on brain structure using voxel based morphometry (VBM) and fMRI in 126 normal volunteers with whole brain and ROI approaches were examined. Phenotypes studied included the $\mathrm{N}$ back and fearful face paradigms using fMRI and optimized VBM for brain structure. Genotypes were also permuted destroying the genotype-phenotype relationship and the analyses repeated. The imaging data were analyzed in SPM2 with a standard alpha of $p=0.05$ corrected for both family wise and false discovery error rates.

For all analyses the rate of positive voxels was well below 5\% (range $0.8-1.4 \%)$ and significantly $(\mathrm{p}<.05$, one sample t test) different from $5 \%$ for all comparisons. There was no relationship between the region of interest and the false positive rate. Since false positive results would have been expected in 5\% of analyses this study provided empirical evidence that the type I error rate is well controlled by commonly used correction procedures in the imaging genetics paradigms used.

Presentation 3: The BDNF val/met polymorphism and hippocampal volume in Dutch Schizophrenia Patients and Controls (Dr. Steven Baker, Utrecht). A study of a Dutch cohort consisting of 75 schizophrenia patients (diagnosed according to DSM-IV criteria) and 97 healthy controls sought to replicate a previous study showing that the BDNF val66met variant has been found associated with reduced hippocampal volume in healthy individuals with more pronounced effect in schizophrenia patients. MRI scans were obtained on a 1.5T Philips scanner. Volumes of the whole brain, intracranium, and hippocampus were determined. The BDNF val/met polymorphism was genotyped by allele specific PCR. The effect of genotype on whole hippocampal volume corrected for age, gender and intracranium was determined using UNPHASED software.

In the combined sample $(\mathrm{n}=172)$ genotype was not associated with hippocampal volume $(\mathrm{p}=.69)$. In schizophrenia patients $(n=75)$ there was a trend towards association $(\mathrm{p}=.08)$ with hippocampal volume. Post hoc testing did not show allelic association $(\mathrm{p}=.21$ in patients; $\mathrm{p}=.85$ in all subjects).

Presentation 4: Gene Discovery through Imaging Genetics: Identification of 2 novel genes for schizophrenia (Dr. Steven Potkin, University of California Irvine). In this study the fMRI activation paradigm of left hemispheric dorsolateral prefrontal cortex activation was used as a phenotype to identify candidate genes for schizophrenia. Two novel genes have been identified for schizophrenia using this method.

The discovery sample $(\mathrm{n}=28$ patients with chronic schizophrenia) was genotyped on the Illumina Human 1 (109,365 gene-centered SNPs) chip and subjects in the verification sample $(n=173)$ on the Human Hap300 (317,503 HapMap tagging SNPs). Significant associations were found on chromosomes 3 and 6 with a circuit involving Brodmann areas 8 and 46, with $\mathrm{p}$ values estimated at $10^{-6}$. Two genes were identified by having at least one SNP whose QT (quantitative trait) analysis was significant at $p<10^{-8}$ with an empirical $p$ value of $10^{-6}$ by permutation. These genes were compared to uncus and hypothalamus (used as control regions) and there was no association.

SNPs related to these two genes in an independent case-control study collected by the FBIRN consortium (www.nbirn.net) were then tested. Fifteen SNPs in these genes were significant. Using a novel genome wide screening strategy with brain activation as the quantitative phenotype two genes have been discovered and verified. Imaging derived neural phenotypes are continuous, quantitative, richer than symptom based diagnoses, and provide considerably more statistical power reflecting the greater penetrance of genetic effects at this more biologically more proximate level.

Presentation 5: (Dr. Patrick Sullivan, Duke University). The Risk of False Positivity. This study in its entirety has been described elsewhere (Wright et al., 2007; Sullivan, 2007). It had three main goals: 1 ) discover the risk of false positives in genetic association studies, 2) understand whether different definitions of replication extinguish vs. propagate false positives, and 3) undertake realistic simulations of a genetic association study using a typical sample size (500 cases and 500 controls) with typical candidate genes.

Realistic simulations via HapSample created 1000 association samples consisting of 500 cases and 500 controls. Genotypes were generated for 10 COMT functional and htSNPs under a null model. All association samples were analyzed for a typical suite of analyses - main effects, main effects stratified by sex, haplotype analyses using a sliding window and five endophenotypes available in cases only. Any positive findings were considered to be false positives by definition. $96.8 \%$ of the studies produced significant findings at $p<.05$ with a median of 6 false positives per study. All positive studies were replicated 10 times. Using a definition of replication as one in which any one SNP was positively associated with any one phenotype, all studies replicated at least 7 times, and $67 \%$ replicated 10/10 times.

Therefore, chance can easily generate findings that seem compelling or intriguing. False positive findings 
can propagate and confuse the literature unless the definition of replication is precise. For the association method to function as intended, every statistical comparison must be tracked and reported, and precise replication (the same SNPs, phenotype, and direction of association) is required.

\section{Discussion}

The emerging field of imaging genetics is exciting but challenging. It is an intersection of 2 fields defined by a high multiplicity of data. Whole Genome Association studies use strategies of increasing sample size and power in relation to small effects.

Several major imaging genetics findings have created great optimism for an intermediate phenotype strategy in psychiatric genetics research. There may be ability to find greater effect on more refined or less complex phenotypes. However, there are several challenges.

1) Imaging genetics studies that use small samples may suffer from genetic heterogeneity since different alleles at the same locus can be associated with disease in populations of different ethnic composition.

2) In using imaging data in disease populations, medication effects on brain structure and function must be considered. Both antipsychotic as well as antidepressant medications can have an effect on brain volume, particularly gray matter. For instance antidepressants may affect BDNF expression, and thus would be important to consider when measuring the effect of the BDNF gene on hippocampal volume, or the effect of antipsychotics on gray matter volumes in general and basal ganglia volume in particular.

3) The selection of statistical correction methods for multiple comparisons is central to an imaging genetics study. Currently FDR and family wise error (FWE) rates are often used. Presentation 2 effectively showed that these methods are adequate for certain types of imaging genetics studies. Bonferonni correction is perhaps the simplest and safest approach for genetics studies involving many tests, as suggested in presentation 5 . However, it is the most conservative approach, and may be overly conservative, since SNPs within genes are not necessarily independent, and neither are voxels or certain brain regions. Another alternative is to use a Monte Carlo method. This approach is well defined in both genetics (Martin and Kaplan, 2000) and imaging (Nichols and Holmes, 2002).

4) Almost implicit in point number 3 , is that of the challenge of replication. Indeed, the 'Winner's Curse' has been well documented in genetics studies of complex disorders (Goring et al., 2001). Ensuring rigorous statistical correction approaches, allows the scientific community at large more certainty in both the original study and attempts at replication. In depth suggestions on what constitutes replication of a genotype-phenotype association and how it can be best achieved have recently been published (Chanock et al., 2007). Further- more, calculating pre-study odds may help improve our understanding of a positive finding (Ioannidis, 2005).

5) The challenge of undertaking a metanalysis was raised. Such an approach is useful in pooling various small studies to increase sample size and examine effect. However, there are many potential confounds to consider in imaging genetics, perhaps more so in imaging. Studies at different centers use different scanners, with different field strengths. Even scanners made by the same company at the same field strength display considerable variability (Littmann et al., 2006). Different software, and different approaches (VBM vs. ROI) methods are used by different centers. It is a great challenge to find a way to combine existing data in a metanalysis.

On the whole, brain imaging is an excellent tool to provide a more objective phenotype, as well as function as an intermediate phenotype 'closer' to the effects of the gene. It provides an opportunity to both discover at risk neural networks in disease, and identify genes that may not show effect at the level of a complex behavioral disorder.

Studies conducted in each discipline, but particularly genetics carry the risk of spurious associations or false positives, that often do not stand the test of replication. The combination of imaging and genetics may lead to more tests requiring correction, but overall the advantages and the biological face validity of using imaging phenotypes in genetics should help accelerate progress in this field.

\section{$\underline{\text { References }}$}

Chanock SJ, Manolio T, Boehnke M, Boerwinkle E, Hunter DJ. 2007. Thomas G, et al. Replicating genotypephenotype associations. Nature 447:655-660.

Goring HH, Terwilliger JD, Blangero J. 2001. Large upward bias in estimation of locus-specific effects from genome-wide scans. Am J Hum Genet 69:1357-1369.

Ioannidis JP. 2005. Why most published research findings are false. PLoS Med 2:e124.

Littmann A, Guehring J, Buechel C, Stiehl HS. 2006. Acquisition-related morphological variability in structural MRI. Acad Radiol 13:1055-1061.

Martin ER, Kaplan NL. 2000. A Monte Carlo procedure for two-stage tests with correlated data. Genet Epidemiol 18:48-62.

Nichols TE, Holmes AP. 2002. Nonparametric permutation tests for functional neuroimaging: a primer with examples. Hum Brain Mapp 15:1-25.

Sullivan PF. 2007. Spurious genetic associations. Biological Psychiatry 61:1121-1126.

Wright FA, Huang H, Guan X, Gamiel K, Jeffries C, Barry WT, de Villena FP, Sullivan PF, Wilfelmsen KC, Zou F. 2007. Simulating association studies: a databased resampling method for candidate regions or whole genome scans. 23(19):2581-2588. 


\section{ENDOPHENOTYPES IN SCHIZOPHRENIA AND BIPOLAR DISORDER Reported by Elizabeth Hare}

Psychiatric illnesses have been difficult to characterize genetically due to their clinical and genetic heterogeneity and the complex interplay of genetic and environmental factors that produce their phenotypes. The analysis of endophenotypes is an approach that provides an alternative method of understanding the genetic basis of these disorders. The essential features of endophenotypes were defined by Gottesman and Gould (2003) as: association with the illness, heritability demonstrated by increased risk for the endophenotype in healthy relatives, state-independence (manifestation whether illness is present or not), and cosegregation with the disease within families. An additional suggested criterion is that the endophenotype is present in family members of individuals with the illness at a higher rate than in the general population.

Dr. Raquel Gur was the Chairperson of the Oral Session on Endophenotypes at the XV World Congress on Psychiatric Genetics. Presentations were given by Drs. Maartje Aukes, Dan Rujescu, Clyde Francks and Michel Maziade. The discussion following these presentations was moderated by Dr. Michael Owen.

The first presentation was by Aukes, who discussed the identification of two useful endophenotypes in the study of schizophrenia: openness and sensorimotor gating. In this study, a battery of neuropsychological and psychophysiological tests was administered to 180 subjects from 25 multigenerational families with multiple members affected by schizophrenia. Statistical analyses included familial correlation for the selection of heritable traits, heritability estimation, and segregation analysis. Moderate familial correlations were found for sensorimotor gating measured with prepulse inhibition, openness, verbal fluency, early visual perception measured with backward masking, and spatial span, with heritabilities estimated from 0.37 to 0.54 . Sensorimotor gating and openness were found to have an inheritance pattern similar to a major gene model with a dominant mode of inheritance, and it was suggested that the other traits were probably the result of more complex modes of inheritance.

Dr Rujescu described a wide range of studies including a scan for association with schizophrenia and cognitive endophenotypes, and gene expression studies. The association study focused on gene regions known to be linked to schizophrenia and candidate genes. The FOXJ1 gene was found to be weakly associated with schizophrenia and executive function, and FOXP1 was associated with memory. The Plexin B3 receptor, another candidate, is a protein that is expressed in the white matter and is involved with neurite outgrowth in axons. It is associated with brain volume, which is highly heritable, and is involved with cognitive function but not schizophrenia. A mouse model was described in which disruption of the crystallin CRYBB2 gene lead to altered prepulse inhibition and behavioral alterations. Genetic variations in this gene were associated with cognitive endophenotypes and saccade eye movements in humans.

Dr Francks described findings of a gene, LRRTM1 (Leucine-rich repeat transmembrane neuronal 1), associated paternally with handedness and schizophrenia. Left-right asymmetrical brain function has been implicated in the development of schizophrenia as well as handedness. This work utilized methods including association mapping, analysis of imprinted expression, Northern blotting, methylation analysis and subcellular localization. Association was found between LRRTM1 and a quantitative measure of handedness in siblings with dyslexia when the haplotype of interest is inherited paternally. The same haplotype was found to be overtransmitted paternally to individuals with schizophrenia. By examining the patterns of expression of LRRTM1 in forebrain development, Dr. Francks and his colleagues determined that the protein could influence neuronal differentiation and connectivity. The endophenotype of handedness may be related to schizophrenia and dyslexia and may provide insight into neurodevelopment.

Dr Francks presented evidence for a signal of positive selection upstream of LRRTM1 in the European-descent population, based on analysis of Hapmap data. A member of the audience pointed out that this signal was found only in the European population, and not in the African or Asian populations. He asked if the correct interpretation was that only in the European descent population was it an evolutionary advantage to be righthanded. Dr Francks responded that that the situation was complex and that possibly a new haplotype had arisen in the European population that was protective from psychosis when associated with abnormal brain asymmetry.

The concern was raised by another audience member about whether handedness should be considered an endophenotype for schizophrenia. Was the underlying assumption of this study that there is a single gene for handedness that is related to schizophrenia, or as Dr. Francks suggested, there is more than one gene for handedness, and that the one he focused on was just one of them. There was also mention that paternal transmission of handedness, as suggested by Dr. Francks, was in question, and that large studies of handedness in the population fail to show this. Dr. Francks commented that if multiple genes were involved, others that were not paternally transmitted, it is unlikely that paternal transmission would be detected in epidemiological studies.

The next speaker, Dr. Maziade, described the use of a standard neuropsychological battery with 45 offspring of families affected with schizophrenia or bipolar disorder who were at high risk of developing these conditions themselves. These subjects had at least one 
parent with either schizophrenia or bipolar disorder, and were individually paired with age- and sex-matched controls. High risk offspring showed significant differences from controls in memory and some areas of executive functioning (initiation and planning). Moderate to large effect sizes for IQ and memory were higher than those reported in the literature. It was suggested that there are similarities between the deficits of individuals at risk for schizophrenia and bipolar disorder, and that the differences are mostly in the magnitudes of the impairments rather than the types of impairments found. These effects remained the same when subjects were divided into two age categories, suggesting that these are prodromal traits. It was also suggested that in individuals with particularly high genetic loading for these disorders, the impairment was greater than in a more general high-risk population and that this represents a dose-response relationship between genetic risk loading and level of impairment.

There was a question from the audience regarding some findings in the literature that those at risk for bipolar disorder have similar or better cognitive abilities when compared with controls. Dr. Maziade reiterated that while there were many more studies in families with a high risk of schizophrenia then in families with a high risk of bipolar disorder, they showed a similar profile of impairment with the schizophrenic population having worse performance on cognitive tests than the bipolar population.

The panel discussion following the presentations had a broad focus on the appropriateness of the use of endophenotypes to elucidate the genetic factors in psychiatric conditions. The moderator expressed a need for caution in planning and interpreting studies involving endophenotypes, stating that these studies rest on the assumption that the endophenotype is caused by something that lies on the disease pathway. This is not particularly problematic when endophenotypes are used to find genes; however, when they are used to study the function of genes and how genes lead to disease, it can be misleading. Such associations are difficult, if not impossible, to prove or disprove and the phenomenon of pleiotropy makes it difficult to sort out whether a gene is on the disease pathway or has effects on two (or more) unrelated traits.

In response to these words of caution, several individuals raised the issue of the complexity of a disorder like schizophrenia with its clinical and genetic heterogeneity and the difficulties and controversies about the definition of the disorder. The lack of significant findings for many genome-wide association studies was mentioned as another reason that multiple methods are needed to disentangle the genetic complexity of these disorders. A researcher from Finland suggested using meta-analysis of datasets from different countries. An audience member asked whether it would be possible to use the same core battery of neurocognitive tests world-wide so that meta-analyses could be performed. Another person answered that the same tests are used for most of these studies.

It was suggested that the traits chosen as endophenotypes may be too complex, particularly the results of neurocognitive testing. Some of this testing requires complicated chains of thought and behavior, and it might be more effective to choose simpler traits such as evoked responses measured with EEG. Other suggestions were that psychiatric geneticists work closely with researchers developing animal models to ensure the ability to study detailed aspects of behavior, that the use of medication is taken into account when analyzing neurocognitive data, and that factor analysis could be used with scales of symptoms to produce useful endophenotypes.

\section{Reference}

Gottesman II and Gould TD. 2003. The endophenotype concept in psychiatry: Etymology and strategic intentions. Am J Psychiatry 160:636-645.

\section{GENETICS OF MOOD DISORDERS Reported by Deepak Grover and Ranjana Verma}

The first speaker in this session was Dr. Sven Cichon from The Life \& Brain Center, University of Bonn and title of his talk was "Genetic variation in the gene for brain-expressed tryptophan hydroxylase 2 (TPH2) is associated with bipolar affective disorder". Dr. Cichon highlighted the biochemical function of tryptophan hydroxylase in 5-HT synthesis. 5-HT is involved in a broad range of behavioral functions known to be disturbed in affective disorders, e.g. mood control, sleep, sexual behavior, drug abuse, food intake. Also, low levels of 5-HT are found in patients with depression, suicide victims. The synthesis of $5-\mathrm{HT}$ in brain is controlled by a TPH2 isoform of the gene which is present on chromosome 12 (Walther et al., 2003). The study he carried out was aimed to access the possible contribution of the TPH2 gene in the development of bipolar disorder. The strategy involved resequencing of the coding region of this gene that consists of 11 exons, in 897 bipolar patients and 1335 controls. The sample set comprised of two cohorts, a German sample (649 cases and 1105 controls) and Russian sample (248 cases and 231 controls). The results revealed a haplotype block in the $5^{\prime}$ region of the gene, and a rare coding nonsynonymous SNP rs11178997, significantly associated with the disease. The odds ratio for the haplotype block association was 1.5 and that for the associated SNP was 5.2. The SNP rs11178997 was observed in family history positive patients with BPAD, and evidence for cosegregation with affective disorder was observed in 3 families. The SNP accounts for an amino acid change from hydrophobic proline to polar serine. Homology based prediction suggests the occurrence of this amino acid Pro206 in a highly conserved structural domain of 
the protein. Functional studies demonstrate that this polymorphism leads to reduced solubility and thermal stability, but no significant change in catalytic activity of the enzyme. Another recent study implicates the same promoter polymorphism in reduced TPH2 activity in seretonergic neurons (Scheuch et al., 2007). The speaker concluded by suggesting reduced 5 -HT production in brain as a pathophysiological mechanism of Bipolar disorder. He also stressed the need to investigate both common and rare variations, as the SNP rs11178997 that was detected by resequencing, could have escaped detection based on Hapmap data.

Dr. Randy Blakely from Vanderbilt University School of medicine was the second speaker of the session. His talk was entitled Good riddance to serotonin: molecular regulation and genetics of serotonin transporters. $\mathrm{He}$ highlighted that serotonin transporter (SERT) is the focus of study in the etiology of many mood disorders. It has also been investigated in the course of pharmacogenetic studies as there is evidence that drugs including antidepressants (cocaine and amphetamines) impact SERT activity. His strategy involved identification of structural domains in SERT that lead to neurotransmitter and antagonist specificity using site directed mutagenesis and molecular modeling. They are using site specific antibodies and electrophysiological techniques to evaluate the post-translational mechanism involved in acute carrier regulation in vivo and in vitro. They are also using transgenic mouse models to study the biochemical and behavioral consequences of SERT defects in humans. Their group has identified sites, that when mutated, lead to loss of SSRI and cocaine recognition by the transporter without disrupting its activity. This further lead to the production of a transgenic mice model that was insensitive to these agents in vivo. Through gene regulation studies, they identified two receptors that are linked to protein kinase $\mathrm{G}$ and MAP kinase that fine tune the amount of SERT available for 5-HT clearance (Zhu et al., 2007). They believe that these may serve as novel therapeutic agents. They further carried out molecular genetics investigations to demonstrate that there exist multiple coding SNP variants in SERT that account for regulation of gene activity through these pathways. Dr. Blakely concluded his talk by summarizing the development of a novel set of tools and targets by their group that will be useful in the understanding of involvement of SERT in neuropsychiatric disorders. $\mathrm{He}$ also added that additional identified targets may lead to development of medication to treat 5-HT mediated disorders.

Dr. David C. Glahn from University of Texas Health Science Center made the next presentation entitled Localization of a Quantitative Trait Locus Influencing Serum BDNF Levels in an Isolated Nepalese Population. He mentioned the neurotrophin hypothesis of affective disorders and other evidence that implicate brainderived neurotrophic factor (BDNF) in affective illness
(Martinowich and others 2007). He mentioned that serum BDNF levels are reduced in people suffering from major depression, and the levels seem to increase with the use of anti-depressants. He projected that serum BDNF levels can serve as an important biomarker for many psychiatric illnesses, especially major depression. Their group performed the first family based analysis of genetic determinants of BDNF levels. They used a single large pedigree, consisting of 1070 individuals, of the isolated Jirel population of Eastern Nepal for this investigation. All these individuals were previously genotyped for a $10 \mathrm{cM}$ genome-wide scan. The variance component analysis of the genotype data revealed that serum heritability of BDNF levels was $0.560 \pm 0.047$ $\left(p=6.7 \times 10^{-45}\right)$. Genome-wide quantitative-trait linkage analysis was performed, that localized a single QTL to chromosome $17 \mathrm{p} 11$ region with LOD score of 3.07 with a nominal $p$-value $8.5 \times 10^{-5}$ and genome-wide $p$-value 0.028 . They searched for positional candidates in the linkage region by analysis of transcriptional profiles (Goring et al., 2007) of a database of Mexican American individuals for which serum BDNF levels were previously measured. In the $3 \mathrm{Mb}$ linkage peak, 30 transcripts were identified, of which two genes (UBB and TNFRSF13B) exhibited significant positive association with serum BDNF levels after correction of multiple tests using a false discovery rate of $5 \%$. He then discussed the putative functional relationship of these two genes with BDNF: UBB is involved in proteosomal elimination of cellular waste and is implicated in Alzheimer's disease too, while TNFRSF13B is involved in mediation of inflammation. He concluded that their findings have suggested biological mechanisms that regulate serum $\mathrm{BDNF}$ levels and possible pathways to target for treatment of psychiatric illnesses such as major depression.

The topic of the final talk of the session was Bipolar Association Case-Control study (BACCS): Focus On "Schizophrenia" candidate genes presented by Dr. Darya Gaysina from MRC SGDP Centre, London. She highlighted multiple literature citations that provide evidence for genetic overlap between different psychiatric disorders, especially schizophrenia and bipolar disorder (BD) (Kirov et al., 2005; Craddock et al., 2006). Consistent association of genes such as DISC1, DAOA, DAO, NRG1 and DTNBP1 have been observed with schizophrenia, and suggestive evidence has been found for RGS4 and COMT. The objective of her work was to investigate the genetic association of these schizophrenia candidate genes with BD. A sample set consisting of 1587 individuals including 841 patients with BD and 746 ethnically matched control subjects recruited from sites in London and Toronto were used for the study. SNPlex genotyping system was used to investigate 41 SNPs in total, including DISC1 (8 SNPs), DAOA (9 SNPs), DAO (1 SNPs), NRG1 (8 SNPs), DTNBP1 (7 SNPs), RGS4 (3 SNPs) and COMT (6 SNPs). The results suggest that there is no significant differ- 
ence in allelic or genotypic frequency of any SNP between $\mathrm{BD}$ patients and control individuals. Further, no association was observed for any haplotype constructed between SNPs in these genes also with the BD phenotype. Dr. Gaysina therefore concluded that the results do not indicate the involvement of these polymorphisms with the disease etiology. The sample size used has $92.6 \%$ power to detect an OR of 1.5 for alleles with $20 \%$ frequency. She mentioned that they would like to further analyze the data for gender and clinical-subgroup specific associations.

There were several questions for different speakers and discussion at the end of the session. One question for Dr. Blakely was about the future implications of his work in pharmacogenetics. Dr. Blakely commented that there can be natural variations in genes too for different strains of mice and the findings are indicating which pathways to target rather than which specific genes. Thus, better strategies for SERT manipulation will be helpful in this regard. Dr. Glahn was asked about the correlation of brain and serum BDNF levels, and also why serum levels were used in his study rather than plasma. Dr. Glahn commented that it is important to find correlation between brain and serum levels, and he would want to do that in future, and the reason for using serum levels was simply its availability. No one has been able to measure CSF BDNF because of difficulties obtaining it. It may be possible to use a non-human primate population to investigate this further. Dr. Cichon made a comment about importance of picking rare coding variants for association studies as there is no dense hapmap coverage for many genes of interest in bipolar disorders. Dr. Blakely concluded the session by stressing the importance of functional studies along with genetic studies for finding causative genes.

\section{$\underline{\text { References }}$}

Craddock N, O'Donovan MC, Owen MJ. 2006. Genes for schizophrenia and bipolar disorder? Implications for psychiatric nosology. Schizophr Bull 32(1):9-16.

Goring HH, Curran JE, Johnson MP, Dyer TD, Charlesworth J, Cole SA, Jowett JB, Abraham LJ, Rainwater DL, Comuzzie AG and others. 2007. Discovery of expression QTLs using large-scale transcriptional profiling in human lymphocytes. Nat Genet 39(10):12081216.

Kirov G, O’Donovan MC, Owen MJ. 2005. Finding schizophrenia genes. J Clin Invest 115(6):1440-1448.

Martinowich K, Manji H, Lu B. 2007. New insights into BDNF function in depression and anxiety. Nat Neurosci 10(9):1089-1093.

Scheuch K, Lautenschlager M, Grohmann M, Stahlberg S, Kirchheiner J, Zill P, Heinz A, Walther DJ, Priller J. 2007. Characterization of a Functional Promoter Polymorphism of the Human Tryptophan Hydroxylase 2 Gene in Serotonergic Raphe Neurons. Biol Psychiatry.
Walther DJ, Peter JU, Bashammakh S, Hortnagl H, Voits M, Fink H, Bader M. 2003. Synthesis of serotonin by a second tryptophan hydroxylase isoform. Science 299(5603):76.

Zhu CB, Steiner JA, Munn JL, Daws LC, Hewlett WA, Blakely RD. 2007. Rapid stimulation of presynaptic serotonin transport by $\mathrm{A}(3)$ adenosine receptors. J Pharmacol Exp Ther 322(1):332-40.

\section{IS THERE A GENETIC OVERLAP AMONG THE PSYCHOSES?}

\section{Reported by Emma Dempster}

Current nosology separates the two main disorders of psychosis, bipolar disorder and schizophrenia, into separate disease entities. However there are many similarities between these two disorders that have brought the validity of this dichotomous diagnostic approach into question.

Dr. Nick Craddock prompted us to consider the "rethinking of psychosis" using data from the recent bipolar whole genome association study (Wellcome Trust Case Control Consortium, 2007). In this study one of the most significant SNPs $\left(P=6.2 \times 10^{-5}\right)$ was located in the gene encoding Gamma-Aminobutyric Acid Receptor, Beta-1 (GABRB1), however, when the bipolar cases were split further into those who also made criteria for schizoaffective/bipolar type (SABP) as defined by RDC diagnostic criteria, the significance of this association increased $\left(P=3.8 \times 10^{-6}\right)$. Furthermore, when the allele frequency of the non-SABP was compared against SABP there was a significant difference. This observation stimulated a strategy to be formulated, to test a specific biological system with a refined phenotype. In this case, 100 SNPs across various GABA receptors were genotyped in the case control study using the refined phenotype. A number of GABA subunits and receptors were shown to be associated with SABP: Gamma-Aminobutyric Acid Receptor, Beta-1 (GABRB1); Gamma-Aminobutyric Acid Receptor, Beta-3 (GABRB3); Gamma-Aminobutyric Acid Receptor, Alpha-5 (GABRA5); and Gamma-Aminobutyric Acid B Receptor 1 (GABBR1).

Dr. Craddock went on to comment that the DSM-IV criteria are much more specific than other diagnostic criteria, and perhaps by using this very specific diagnostic criteria we are missing shared underlying biological processes that may be best delineated by using a broader phenotype. He speculated that the two main psychosis disorders, schizophrenia and bipolar disorder, fall on the opposite ends of a spectrum and by looking at phenotypes that represent "middle ground" we may be able to elucidate shared underlying biological processes between the two disorders. However, one of the main stumbling blocks of this "middle ground" is that it is not consistently diagnosed. 
Dr. Craddock concluded by proposing a move away from the 19th century Kraeplin categorical approach to diagnosis to a more 21st century system based on biological systems. He did, however, stress that the association between the GABA genes and broad schizoaffective disorder is not going to change diagnosis but lead to the identification of target biological systems. Linking a specific gene to one particular symptom is unfeasible, but by combining fastidious clinical workups, neuropsychiatry testing and laboratory tests of course including genetics will be the key to better diagnosis.

Alexander Niculescu applied his convergent functional genetics approach to tackling the overlap in psychiatric disorders. This method uses a Bayesian strategy to cross-interrogate large data sets to prioritize candidate genes and pathways and identify novel biomarkers. This method makes use of quantitative data from a variety of sources, e.g. linkage data, microarray expression analysis and convergent pharmacological studies in animals and humans in parallel. He likens his approach to the internet search engine "Google", the more information on one gene the higher it is ranked. Niculescu has successfully applied this approach to three of the main psychiatric disorders, schizophrenia (Le-Niculescu et al., 2007a), bipolar (Ogden et al., 2004; Le-Niculescu et al., 2007b) and alcoholism (Rodd et al., 2007). By examining the overlap of all these datasets 4 candidate genes, D Site Of Albumin Promoter-Binding Protein $(D B P)$; Adenosine A2 Receptor (ADORA2A); Myelin-Associated Oligodendrocyte Basic Protein (MOBP); and Coagulation Factor III (F3), were found in common with schizophrenia, bipolar, anxiety disorder and alcoholism. Recently they have extended their approach to investigate peripheral biomarkers present in the blood and have preliminary evidence that brain specific genes are heterogeneously expressed in the blood and have the potential to be used as biomarkers.

Concentrating more on the specific overlap in the psychoses Dr. Niculescu introduced his PhenoChipping strategy (Niculescu et al., 2006). This method, which uses tools first developed to analyze gene expression microarray data to investigate correlations between phenotypes and different quantitative psychiatric scales, was applied to determine the extent of phenotypic overlap between schizophrenia, bipolar and schizoaffective disorder. The resulting conclusion is that, although heterogeneity in phenotypes exists between the three diagnoses, there is a substantial overlap of symptoms, which is not currently addressed in the current diagnostic scales available.

Alexander Georgi presented his work on premorbid adjustment in the psychosis. Generally, much of the research in this area has focused on schizophrenia, which has found that premorbid adjustment is worse in individuals who go on to develop schizophrenia and can be a predictor of clinical presentations and outcome
(Schmael et al., 2007). When Dr. Georgi and colleagues investigated premorbid adjustment in bipolar disorder they found a stark contrast to the picture seen in schizophrenia, in that bipolar patients had excellent premorbid functioning and were actually better adjusted than the control sample. Furthermore, no difference was seen between bipolar patients with psychosis and those without. These observations appear to challenge the notion that schizophrenia and bipolar psychosis share an underlying biology.

William Hennah reported on a recent collaborative study on one of the most promising candidate genes in schizophrenia and bipolar disorder, DISC1. This gene was first identified from a chromosomal translocation found to be segregating with a broad diagnosis of psychiatric illness in a large Scottish pedigree (St Clair et al., 1990 ) Since this first report there has been a number of linkage and association studied in diverse populations that support DISC1 as a susceptibility locus for a wide range of psychiatric phenotypes including bipolar disorder and schizophrenia but as yet a functional variant has not been uncovered and many of the studies have little overlap and there has been very few direct replications.

Dr. Hennah genotyped 75 SNPs in a number of different European populations (consisting of 1295 schizophrenics and 1262 controls; and 994 bipolar patients and a further 1004 controls). 60 of the SNPs genotyped tagged 20 haplotype blocks across the DISC 1 region and an additional 15 SNPs were included that had putative functionality previously implicated.

Significant association, between bipolar and combined bipolar disorder and schizophrenia, and SNP rs1538979 was identified in a Finnish sample. Meanwhile, another SNP, rs821577 was found to be associated with bipolar disorder in a London sample. Allele frequency heterogeneity existed between European populations for SNP rs1538979, but not for rs821577.

The European cohorts were then divided up depending on if an individual was carrying the risk variant for rs1538979 or rs821577. This led to the identification of a further SNP, rs821633 that was significantly associated with schizophrenia in a subgroup that also carried the risk allele of rs1538979. Further modeling on the combination of different risk variants led to the conclusion that if you only carry the rs1538979 or rs 821577 "risk" variants they are neutral of risk; meanwhile, if you only carry the rs821633 "risk" allele it decreases risk. But if you have a combination of rs821633 and either or both of rs 1538979 or rs 821577 , then it increases risk. This study highlighted the issue of allele frequency heterogeneity in differing populations and psychiatric phenotypes.

The discussion that followed the sessions focused on the viability of the current DSMIV diagnoses in genetic research. The main conclusion drawn was that the current nature of DSMIV diagnosis is unsatisfactory and that a more modular diagnostic approach would be 
better suited to elucidating the underlying biological processes. Currently, in psychiatric genetics there is a common problem of non-replication, therefore not only are we faced with allele frequency heterogeneity between populations, but due to differences in diagnosis there is considerable phenotypic heterogeneity. The panel agreed that a concerted effort needs to be made to better describe samples clinically to enable meaningful replication. The main recommendation from the panel discussion was that as a community we should make our voice heard at the international bodies responsible for the current diagnostic criteria.

\section{$\underline{\text { References }}$}

Wellcome Trust Case Control Consortium. 2007. Genome-wide association study of 14,000 cases of seven common diseases and 3,000 shared controls. Nature 447(7145):661-678.

Le-Niculescu H, Balaraman Y, et al. 2007. Towards understanding the schizophrenia code: an expanded convergent functional genomics approach. Am J Med Genet B Neuropsychiatr Genet 144(2):129-158.

Le-Niculescu H, McFarland MJ, et al. 2007. Convergent Functional Genomics of bipolar disorder: from animal model pharmacogenomics to human genetics and biomarkers. Neurosci Biobehav Rev 31(6):897-903.

Niculescu AB, Lulow LL, et al. 2006. PhenoChipping of psychotic disorders: a novel approach for deconstructing and quantitating psychiatric phenotypes. Am J Med Genet B Neuropsychiatr Genet 141(6):653-662.

Ogden CA, Rich ME, et al. 2004. Candidate genes, pathways and mechanisms for bipolar (manic-depressive) and related disorders: an expanded convergent functional genomics approach. Mol Psychiatry 9(11): 1007-1029.

Schmael C, Georgi A, et al. 2007. Premorbid adjustment in schizophrenia-an important aspect of phenotype definition. Schizophr Res 92(1-3):50-62.

St Clair D, Blackwood D, et al. 1990. Association within a family of a balanced autosomal translocation with major mental illness. Lancet 336(8706):13-16.

\section{NEW GENETIC APPROACHES TO STUDIES OF DEMENTIA Reported by Lan Yu}

\section{Chair: Hiroshi Yoneda Discussion Moderator: Mony De Leon}

Five presentations highlighted this session. Dr. Mony De Leon from New York University gave an overview of brain imaging and genetics in Alzheimer's Disease $(\mathrm{AD})$, highlighting some of the results from his laboratory. These data showed hippocampus and entorhinal cortex progressively reducing in size while the temporal horns of the lateral ventricle were expanding in size in individuals who progress from mild cognitive impair- ment (MCI) to AD. However, they also found evidence for hippocampal atrophy with increasing age in the normal elderly population, with the hippocampal atrophy in $\mathrm{MCI}$ and $\mathrm{AD}$ subjects to be age independent. In addition, positron emission tomography (PET) consistently demonstrated a temporo-parietal metabolic defect in patients with $\mathrm{AD}$ and that these changes may be predictive of who converts from MCI to AD. Several studies examined the relationship between brain imaging findings and gene mutations. Familial Alzheimer's disease (FAD), an early onset $\mathrm{AD}$ with autosomal dominant inheritance was studied by Mosconi and colleagues in his group. Seven presymptomatic individuals from FAD carrying mutations in the Presenilin 1 gene were found to have reduced glucose metabolism on PET that distinguished them from controls, suggesting that PET measures may serve as biomarkers for the preclinical diagnosis of AD. Furthermore, the pattern of metabolic reduction in individuals with the Presenilin 1 gene mutation is consistent with reports on presymptomatic individuals who are carriers of the (apolipoprotein) ApoE 4 allele. When examining the effects of ApoE genotype on subjective memory complaints (SMC) and cerebral metabolic rates on PET, Mosconi et al found that E4 carriers with SMC (E4+/SMC+) showed significant PET alterations compared with E4 negative individuals, concluding that the ApoE E4 genotype is not causative but facilitates the metabolic decline, although the mechanism is unknown. Dr. Andrew Saykin focused on a Genome Wide Association Study Using Medial Temporal Lobe Morphology on MRI as the endophenotype with a Targeted Pathway Array in older adults atrisk for Alzheimer's Disease. He commented that in the future neuroimaging in combination with genetics will become important biomarkers for early detection and medication response in clinical trials. His work is attempting to examine the memory decline in older adults particularly in those patients with $\mathrm{AD}$, and to explore the boundary between normal and pathological aging. Adults enrolled in a longitudinal study of memory and aging ( 40 healthy controls, $40 \mathrm{CC}$ and $40 \mathrm{MCI}$ ) were characterized and studied with neuropsychological testing and MRI. A genotyping array (Affymetrix MIP) was designed to assess candidate SNPs for genes in the following categories: neurodegeneration, neuroinflammation, neurotransmitter receptors, cerebrovascular and metabolic/endocrine factors, plasticity/repair and growth factors and the molecular substrates of cognition and memory/long term potentiation. The CC (cognitive complaint) and MCI groups showed similar decreased gray matter density relative to the $\mathrm{HC}$ group on whole brain analysis. Manually segmented hippocampal volumes were significantly reduced only in the MCI group. Significant Dx by SNP interactions with $\mathrm{p}<.001$ were observed in multiple candidate pathways. Particularly notable were associations with hippocampal volumes ( $\mathrm{R}$ more frequent than $\mathrm{L}$ ). A subset surviving stringent multiple testing corrections and threshold 
issues are being examined in ongoing statistical analyses. He concluded that allelic variation in targeted pathways accounts for significant variance in hippocampal morphology in those at risk for $\mathrm{AD}$. Medial temporal lobe gray matter density appears more sensitive than volume to early preclinical stages (i.e., cognitive complaint group), however hippocampal volume shows a greater indication of genetic influence. Future directions are to look for genetic predictors of hippocampal volume changes and to explore polymorphisms that relate to changes in brain activation after medication. The specific pathways and alleles, however, were not specified, as the final data analyses are still in progress. One issue that arose in the discussion was whether the same genes that influence hippocampal volume in $\mathrm{AD}$ have an influence in hippocampal reduction in other conditions, such as schizophrenia. He concluded that there may be a final common pathway for many etiologies to have an effect on this venerable structure, but that a genetic overlap between schizophrenia and $\mathrm{AD}$ was unlikely.

The third speaker, Dr. Gary Beecham reported on another whole-genome association study using LateOnset Alzheimer Disease (LOAD) as the phenotype. LOAD is the most common cause of dementia and there are over 5 million individuals affected with LOAD in the US. The lifetime risk is $10-15 \%$ in the general population and the prevalence doubles every 5 years after age 60. Genetic studies have estimated the heritability of LOAD to be $80 \%$, however, APOE is the only consensus LOAD risk gene which accounts for at most $50 \%$ of the genetic effect. Thus, a genome-wide association (GWA) study for $\mathrm{LOAD}$ may be useful to detect other $\mathrm{AD}$ risk loci. Beecham's group used the Illumina Infinium platform to genotype 518 cases and 531 controls for 550,000 SNPs. They implemented numerous quality control tests to ensure the integrity of the data, and tested for association using the Armitage's Trend test. One SNP on chromosome 12 (rs11610206) and three APOE SNPs were significantly associated at the 0.05 level (multiple testing corrected). Rs11610206 lies directly under the narrow covariate-based linkage peak on $12 \mathrm{q}$ and is located near the Vitamin D Receptor (VDR) gene previously associated with AD. Thus, these results provide convincing evidence for an $\mathrm{AD}$ risk locus on $12 q 13$.

The fourth speaker, Dr. William Kremen, spoke on the effects of the ApoE genotype on brain and cognition in the sixth decade of life. Much research suggests that signs of $\mathrm{AD}$ are present long before it can be diagnosed. The ApoE genotype appears to be a risk factor for $\mathrm{AD}$. Almost all adult studies of ApoE genotypes and cognition include individuals over 60 . In older adults, E4 carriers may manifest increased cognitive asymmetry. Moreover, general cognitive ability may be affected by both aging and ApoE genotype, but most studies inadequately address this potential confound. To examine the relationship of $\mathrm{ApoE}$ genotype to episodic memory, verbal-visual-spatial episodic memory asymmetries, and selected brain structures, he and his colleagues compared E4+ and En4- in 626 male twins in their 50s. They also compared verbal and visualspatial episodic memory and verbal-visual asymmetry scores after adjusting for cognitive ability at age 20 . Compared with E4- individuals, E4 carriers performed more poorly on verbal, but not visual-spatial memory, manifested greater cognitive asymmetry, and had more concerns about memory. At age 20, E4 carriers had higher general cognitive ability than E4- individuals, and current memory differences were enhanced after adjusting for age 20 cognitive ability. Brain caudate and putamen volumes were smaller in E4 carriers, but they had less white matter abnormalities. Results strongly suggest that APOE-4 related impairments are risk factors for dementia, but not just signs of prodromal $\mathrm{AD}$.

The final presentation was given by Dr. Ryo Kimura who focused on the DYRK1A gene, encoded in the chromosome 21 Down syndrome critical region. This gene bridges beta-amyloid production and tau phosphorylation in AD. Dr. Kimura and his research group scanned chromosome 21 for genetic associations to LOAD using 374 Japanese trisomy 21 patients and 375 population-based controls, because trisomy 21 is known to be associated with early deposition of $\boldsymbol{\beta}$-amyloid (A $\boldsymbol{\beta}$ ) in the brain. Among 417 markers spanning $33 \mathrm{Mb}, 22$ markers showed associations with either the allele or the genotype frequency $(\mathrm{P}<0.05)$. Logistic regression analysis with age, sex and ApoE-epsilon4 dose supported genetic risk for 17 markers, of which eight markers were linked to the SAMSN1, PRSS7, NCAM2, RUNX1, DYRK1A and KCNJ6 genes. In logistic regression, the DYRK1A (dual-specificity tyrosine-regulated kinase 1A) gene, located in the Down syndrome critical region, showed the highest significance $[\mathrm{OR}=2.99(95 \%$ CI: 1.72-5.19), $\mathrm{P}=0.001]$. DYRK1A mRNA level in the hippocampus was significantly elevated in patients with $\mathrm{AD}$ when compared with pathological controls $(\mathrm{P}<0.01)$. In addition, DYRK1A mRNA levels were increased with A-beta loading in the brains of transgenic mice at 9 months of age. In human neuroblastoma cells, A-beta induced an increase in the DYRK1A transcript, which also led to tau phosphorylation at $\mathrm{Thr}^{212}$ with overexpression of tau. Therefore, the upregulation of the DYRK1A transcription likely results from Abeta loading, further leading to tau phosphorylation. They concluded that DYRK1A could be a key molecule bridging beta-amyloid production with tau phosphorylation in $\mathrm{AD}$.

\section{NEW PERSPECTIVES ON SCHIZOPHRENIA CANDIDATE GENES Reported by Anna Alkelai}

Introduction: There are four main strategies to search for genes for schizophrenia: studies of chromosomal 
abnormalities, genetic linkage studies, association studies and convergent genomics (Owen et al., 2005). Recently the field of schizophrenia genetics has focused on association studies using functional or positional candidate gene approaches. In this session five speakers presented their work based on association studies, with the main goal of identifying schizophrenia susceptibility genes.

Presentations: The first presenter was Dr. Nigel Williams. 22q11 deletion syndrome is the most common known microdeletion in humans. It is also the most common known genetic risk factor for schizophrenia (Gothelf et al., 2007). The previously published study by Paylor et al. (2006) revealed two strong candidate genes (TBX1/GNB1L) for psychiatric disease in this $22 \mathrm{q} 11$ region. The main goal of Dr. Williams's study was to test the hypothesis that variants in these two genes confer susceptibility to develop psychosis. In this study the association analysis of SNPs within GNB1L and TBX1 genes was performed in a schizophrenia case control sample and was replicated in two independent schizophrenia case control samples and also in schizophrenia parent proband trios. Significant male specific genotypic association was observed in all replication samples. In addition, it was shown that the most associated SNP was correlated with expression of the GNB1L gene. The SNPs showing the strongest association in males in schizophrenia case/control samples was also associated with psychosis in an additional male 22q11DS sample. These data suggest that increased risk of developing psychosis results from genetic variation at $T B X 1 /$ $G N B 1 L$ locus and is mediated in a dosage sensitive manner possibly via gene expression.

The second presenter was Dr. Jubao Duan. A previously published genome-wide linkage scan of 409 families with schizophrenia revealed suggestive evidence of linkage at chromosome 8p23.3-p21.2 (Suarez et al., 2006). In Dr. Duan's research a dense LD mapping association study of this region was performed in a large schizophrenia and schizoaffective disorder case control sample. Nominally significant association was detected for several SNPs in or near the CSMD1, MFHAS1, PSD3 or EBF2 genes with the best result for SNPs near $E B F 2$. In addition, copy number variants in the same sample were analyzed and a rare $72 \mathrm{~kb}$ deletion in MSR1 was found to be overrepresented in cases with nominal significance. Genotyping of additional SNPs in the region did not improve the significance. It was assumed during discussion that if all the results are true positives, then a number of nominally associated genes, each one probably with small effect, could be involved in schizophrenia susceptibility.

The third presenter was Dr. Anna K Kahler, who studied the putative association between schizophrenia and gene variants involved in the neuronal migration process, in three Scandinavian case control samples. There is evidence in the literature that schizophrenia is a neurodevelopmental disorder, and brain abnormal- ities in patients with schizophrenia could be due to an abnormal neuronal migration (Weickert et al., 1998). SNPs in 18 genes were genotyped and nominally significant association for several SNPs in both the RELN gene and a gene encoding an adhesion molecule was identified. It was mentioned during the presentation that genotyping of additional SNPs could be done to improve significance. This study presented additional support for the previously reported schizophrenia candidate RELN and suggested a possible new candidate gene.

The fourth presenter was Dr. Stephen Glatt. Prior case control studies found a reliable effect of one DRD2 polymorphism on risk for schizophrenia (Arinami et al., 1994). In Dr. Glatt's study the first family-based association study of schizophrenia with haplotypetagging and all three known non-synonymous polymorphisms in DRD2 was performed. Significant evidence even after Bonferroni correction was found for one polymorphism and two haplotypes. These results further strengthen the candidacy of DRD2 as a schizophrenia risk gene.

The fifth presenter was Dr. Masashi Ikeda. Prior studies identified Neuregulin1 as a candidate gene for schizophrenia (Stefansson et al., 2002). In Dr. Ikeda's study three-stage association analysis with tagging SNPs in Neuregulin1 gene was performed in a large Japanese sample. This study did not replicate the association between the Neuregulin1 gene and schizophrenia susceptibility in the studied population.

Discussion: It was noted during the session discussion that replications in other independent samples are needed to confirm the results of the presented studies, and that it is essential to combine association with functional analysis. It was also suggested that it may be of benefit to re-genotype and combine samples which were used in early studies, since many aspects have been improved since then: sample sizes have increased, genotyping technology has improved, and knowledge from the human genome project has accumulated. It was also mentioned that the discovery of susceptibility genes for schizophrenia in the future will hopefully improve the understanding of the disease, will propose more efficient treatments, and will help to find individuals at risk to be affected with genetic testing. Since the clinical implications of genetic findings are not yet clearly visible, psychiatric genetics and medical clinic still work as independent fields. It was proposed during the session discussion that it may be too conservative to wait for the clear and obvious genetic findings. It was proposed that physicians have to be more involved in genetic research field and some clinical implications should be considered.

\section{References}

Arinami T, Itokawa M, Enguchi H, Tagaya H, Yano S, Shimizu H, Hamaguchi H, Toru M. 1994. Association of 
dopamine D2 receptor molecular variant with schizophrenia. Lancet 343:703-704.

Owen MJ. 2005. Genomic Approaches to Schizophrenia. Clin Ther 27 Suppl A:S2-S7.

Gothelf D. 2007. Velocardiofacial syndrome. Child Adolesc Psychiatr Clin N Am 16(3):677-693.

Paylor R, Glaser B, Mupo A, Ataliotis P, Spencer C, Sobotka A, Sparks C, Choi CH, Oghalai J, Curran S, Murphy KC, Monks S, Williams N, O'Donovan MC, Owen MJ, Scambler PJ, Lindsay E. 2006. Tbx1 haploinsufficiency is linked to behavioral disorders in mice and humans: implications for $22 \mathrm{q} 11$ deletion syndrome. Proc Natl Acad Sci USA 103(20):7729-7734. Stefansson H, Sigurdsson E, Steinthorsdottir V, Bjornsdottir S, Sigmundsson T, Ghosh S, et al. 2002. Neuregulin 1 and susceptibility to schizophrenia. Am J Hum Genet 71(4):877-892.

Suarez BK, Duan J, Sanders AR, Hinrichs AL, Jin CH, Hou C, Buccola NG, Hale N, Weilbaecher AN, Nertney DA, Olincy A, Green S, Schaffer AW, Smith CJ, Hannah DE, Rice JP, Cox NJ, Martinez M, Mowry BJ, Amin F, Silverman JM, Black DW, Byerley WF, Crowe RR, Freedman R, Cloninger CR, Levinson DF, Gejman PV. 2006. Genomewide linkage scan of 409 Europeanancestry and African American families with schizophrenia: suggestive evidence of linkage at 8p23.3-p21.2 and 11p13.1-q14.1 in the combined sample. Am J Hum Genet 78(2):315-333.

Weickert CS, Weinberger DR. 1998. A candidate molecule approach to defining developmental pathology in schizophrenia. Schizophr Bull 24(2):303-316.

\section{THE GENETICS OF SUBSTANCE ABUSE Reported by Marlon P. Quinones}

Substance abuse is now recognized as a chronic, relapsing disease characterized by compulsive drug seeking. Drug addiction costs to our nation more than $\$ 484$ Billion per Year ${ }^{1}$. Over half $(51 \%)$ of America's teenagers have tried an illicit drug by the time they finish high school. In 2003, an estimated 71 million Americans reported being current users of a tobacco product, 14.6 million people were current users of marijuana, and nearly 17.6 million adults in the United States are alcohol-dependent or have problem with alcohol. Drug addiction shares many features with other chronic illnesses, including a tendency to run in families (heritability), an onset and course that is influenced by environmental conditions and behavior, and the ability to respond to appropriate treatment, which may include long-term lifestyle modifications.

Indeed, human studies of addictive behaviors have clearly implicated environmental, genetic influences and their interactions in disease pathogenesis. All these pathogenic factors were addressed in the ISPG's meeting session on genetics of substance abuse. The session

\footnotetext{
${ }^{1}$ Data source: National Institute on Drug Abuse (NIDA) website.
}

chair was Dr. Ming Tsuang, Professor of Psychiatry, University of California, San Diego, and Director, Institute of Behavioral Genomics. The discussion moderator was Dr. Kenneth Kendler, Professor of Psychiatry Virginia Institute for Psychiatric and Behavioral Genetics, Virginal Commonwealth University.

The first talk in the session addressed the need for the development of relevant animal models to study the mechanisms by which genes affect the susceptibility to substance abuse. In his talk, Dr. Eric Vallender presented research demonstrating how investigators can use rhesus monkeys to dissect the effects of single nucleotide polymorphisms (SNPs) on the function of genes whose products have a critical role in neurotransmitter systems. In principle, rhesus monkeys with overlapping yet distinct constellations of disorderrelated alleles that mimic in effect orthologous human alleles could be utilized to both clarify the genetic interactions influencing disorder-related phenotypes as well as serve as a preclinical platform for the development of much-needed human pharmacogenomics-based treatment interventions. Some of the examples presented in the session included SNPs in the Tryptophan hydroxylase (TPH)-2 gene, a second form of TPH responsible for serotonin synthesis in the brain. SNPs in the TPH2 have been increasingly implicated as contributors to the etiology of various psychiatric disorders. Notably, the rhesus' TPH2 gene also has functional SNPs. Dr. Vallender also commented on other functional polymorphisms identified on the rhesus' serotonin transporter and monoamine oxidase-type a genes. He postulated that collectively, polymorphisms in these genes are likely to influence substance abuse and that we can understand the mechanisms underlying their effects using rhesus monkeys. An interesting question from the audience was whether rhesus monkeys have a degree of genetic variability as great as that seen in humans. Dr. Vallender commented that there seem to be extremely high degree of polymorphisms in the Indian macaques that the authors have used for their studies and that aids in the use of these animals as genetic models for the detection of genetic variability in susceptibility to substance abuse.

The next talk described new data on the genetic variation of specific genes and the risk for alcohol dependence. The rational for this work is provided by data from twin, family, and adoption studies demonstrating a major genetic influence on vulnerability to alcoholism. Dr. John Nurnberger, from Indiana University School of Medicine, presented data derived using large, genetically informative pedigrees from the Collaborative Study on the Genetics of Alcoholism (COGA) (Dick et al., in press). Since 1989 COGA has been federally funded aiming to identify specific genes underlying vulnerability to alcohol dependence. The COGA investigators, in the course of carrying out their linkage studies of alcoholism, have assembled a collection of $>300$ extended families densely 
affected by alcoholism, consisting collectively of $>3000$ individuals. They have collected extensive clinical, neuropsychological, electrophysiological, biochemical, and genetic data, and established a repository of immortalized cell lines from these individuals, to serve as a permanent source of DNA. Dr. Nurnberger's broadly commented on specific single genes implicated in the susceptibility to alcohol abuse including acetate nonutilizing (ACN)-9, gamma-aminobutyric acid receptor A alpha-2 subunit (GABRA2; Dick et al., 2006a and b), alcohol dehydrogenase (ADH)-4 (Edenberg et al., 2006), cholinergic receptor muscarinic (CHRM)-2(Wang et al., 2004; Jones et al., 2004), -5, glutamate receptor, metabotropic (GRM)-8; and how information about these gene-candidates, was derived using different, but equally powerful methodology for the dissection of the genetics of alcohol dependence. Some of approaches included saturation genotyping with SNP's in a linkage region, an endophenotype-based approach, or a "Convergent Functional Genomics" approach that crossmatches gene expression data from rodent models with human genetic linkage data for the preliminary identification of gene-candidates.

An example of the endophenotype approach relates to the studies on GRM8 as a candidate gene. Due to the richness of data collected in the COGA cohort, it is possible to look for more proximal phenotypic correlates of gene variation (i.e., endophenotypes), which collectively are thought to be associated with alcohol dependence. Evidence suggests the P3 amplitude of the eventrelated potential and its underlying superimposed event-related oscillations, primarily theta $(4-5 \mathrm{~Hz})$ and delta $(1-3 \mathrm{~Hz})$ energy bands, are endophenotypes for the risk of alcoholism and other disinhibitory disorders. Major neurochemical substrates contributing to theta, delta rhythms and P3 involve strong GABAergic, cholinergic and glutamatergic system interactions. GRM8 was selected as candidate because it maps at chromosome 7q31.3-q32.1 under a peak region previously identified as having significant linkage using a genome-wide linkage scan. It was reported that using a family based association test, there was a significant genetic association between multiple SNPs in the GRM8 gene and event-related theta power to target visual stimuli. This was the case even after correction for multiple comparisons by false discovery rate (FDR). Base on these results it was concluded that variation in GRM8 could be involved in modulating event-related theta oscillations during information processing and in vulnerability to alcoholism.

Dr. Nurnberger also commented on interesting results related to other genes linked to alcohol dependence identified using other approaches. For instance, mice with a homozygous deletion the clock gene (and a transcription factor) D-box Binding Protein (DBP) were found to have a baseline lower locomotor activity, blunted responses to acute methamphetamine administration, and gain less weight over time compared to wild-type controls, analogous to DSM criteria of depression in humans. In response to a chronic stress paradigm, DBP KO mice exhibit a diametral switch in these phenotypes, resembling phenotypic aspects of bipolar disorder. Notably, level of DBP in the frontal cortex differs between alcohol preferring vs. non-alcohol preferring rats. Together, the data available so far suggested that DBP could be an important candidate for alcoholism and co-morbid psychiatric disorders. Dr. Nurnberger mentioned recently published associations between (i) alpha synuclein and alcohol craving, a common, although not uniform, feature of alcohol dependence; (ii) dynorphin, mu-opioid receptor, GABRA2 CHRM2 and alcohol dependence; and (iii) GABRA1 with several of the drinking behavior phenotypes, including COGA alcohol dependence, history of blackouts, age at first drunkenness, and level of response to alcohol.

The next speaker continued with this line of research. Dr. Brien Riley, from the Departments of Psychiatry and Human Genetics and Molecular Laboratory at Virginia Institute for Psychiatric and Behavioral Genetics, described results of fine-mapping of a $18 \mathrm{mb}$ alcohol dependence susceptibility locus on 4q22-q32 in the Irish Affected Sib-pair Study of Alcohol dependence (IASPSAD). This study includes 1414 individuals from 591 families ascertained in Ireland. Alcohol dependence was assessed among 1201 probands and affected siblings with use of the Semi-Structured Assessment for the Genetics of Alcoholism and among 213 parents with use of a modified version of the Structured Clinical Interview for DSM. SNPs in a number of genes in the region showed modest evidence of association with the diagnosis of alcohol dependence. The strongest association signal observed for the $\mathrm{AD}$ diagnosis was with SNPs in the neuronal ankyrin 2 (ANK2). Several lines of evidence support this putative association. First, a related gene, ankyrin repeat and kinase domain containing 1 (ANKK1), was recently reported associated with $\mathrm{AD}$ in the COGA sample. Second, ankyrins are thought to link membrane-bound proteins to the underlying spectrinactin cytoskeleton, and Eps8 knockout mice show resistance to both the acute intoxicating effects of ethanol and ethanol-mediated actin remodeling. Finally, Mendelian mutations in ANK2 cause long-QT syndrome cardiac arrhythmia. The QT interval is prolonged in alcoholics (and this can persist in abstinence) and acute ethanol increases the QT interval in controls. ANK2 also showed association with multiple phenotypes related to $\mathrm{AD}$, including nicotine dependence.

However, the chromosome 4q linkage in this sample was strongest when analyzing a quantitative trait of number of $\mathrm{AD}$ symptoms endorsed (ADSx). A different locus, the dickkopf homolog 2 (DKK2) gene gave strongest evidence for association with $\mathrm{ADSx}$, and was specific to the ADSx phenotype. DKK2 has not previously been implicated in alcohol-related phenotypes.

Completing the series of genetic association studies focused on the genetic basis of alcohol dependence, 
Dr. Danielle Dick, Departments of Psychiatry, Psychology, and Human Genetics, Virginia Institute for Psychiatric and Behavioral Genetics at Virginia Commonwealth University, presented data on a systematic screen of a linkage region on chromosome 7 in the COGA study. A panel of 1536 SNPs were genotyped to tag linkage disequilibrium bins across a 2 LOD support interval around the alcohol dependence linkage peak on chromosome 7. Four of the eight most significant SNPs from the screen were all located in or very near the ACN9 gene. Additional genotyping across ACN9 was conducted and identified multiple variants with significant evidence of association with alcohol dependence. Little is known about the role of ACN9 in humans; Data from yeast suggest that ACN9 is involved in acetate utilization and gluconeogenesis. This may suggest a plausible role for ACN9 in the predisposition to alcohol dependence (Dick et al., in press).

Complementing the work presented by other speakers on the identification of genetic determinants of substance abuse, Dr. Kendler spoke on gene-environment interactions. His group used retrospective assessments of life history from 1,796 male twins, aged 24-62 from the population-based Virginia Twin Registry. Dr. Kendler's data suggest that in early adolescence, twin resemblance for nicotine, alcohol and cannabis use was entirely environmental in origin. Nevertheless, with age, genetic effects became increasingly important in influencing substance use while shared environmental effects diminished and then disappeared. But, what kind of environmental influences have such a powerful influence in the susceptibility to substance abuse? Preliminary evidence suggests that peer group deviance; drug availability and parental monitoring substantially mediate shared environmental effects on psychoactive substance use.

Indeed, a very fascinating aspect of Dr. Kendler's work relates to his research on peer group deviance. To conduct research on peer deviance, twins reported how many friends they had had in each time period who smoked, drank, cut classes often, used or sold drugs, stole things, or got in trouble with the law (collectively indicators of deviance). Then, concordance rates between monozygotic and dizygotic twins in the likelihood of choosing the same kind of friends (endorsing more or less deviance indicators) were calculated. Given that there was a higher concordance rate between monozygotic and dizygotic twins, the data suggested that there was a genetic inclination to select certain types of people as their friends. Remarkably, this finding is closely linked with susceptibility to substance abuse. The genetic inclination to choose peers that use substances could increase risk by determining the levels of exposure substances. Regarding the modulatory effects of age, estimates from Kendler's work revealed that for 8-11 year old children, genes explained $30 \%$ of their choice in friends, with that percentage rising to about $50 \%$ from age $15-25$, as people matured into independent adults. These data suggest that as individuals grow older, genetic factors play an increasingly more important role in the social world that they create for themselves.

Another critical factor addressed by the speaker was the inter-relationship between personal deviancyassessed by symptoms of conduct disorder (CD) - and peer group deviance. It was suggested that genes could act to influence $\mathrm{CD}$, which through social selection impacts on peer group deviance. Shared environment could impact on peer group deviance, which, via social influence, alters risk for CD.

From the discussion that followed Kendler's work, and as part of the final discussion of the material presented in the session, it became clear that there is a great interest on defining possible genes that influence personal and peer group deviance; as well as to model gene and environment interactions. The studies that have been conducted up to date have begun to reveal the genetic underpinnings of substance abuse. Animal models are extremely powerful helping to identify candidate genes and allowing testing of the mechanisms by which genetic variation influence gene function. There seemed to be a consensus that future studies should take into account environmental influences, and to examine the possible genetic determinants of substance abuse as dynamic factors, having effects that may be of importance only at specific developmental stages. Indeed, more research is needed to determine whether social factors determine initial exposure to substances of abuse while genetic determinants influence persistence use and dependency.

\section{$\underline{\text { References }}$}

Dick DM, Aliev F, Wang JC, Saccone S, Hinrichs A, Bertelsen S, Budde J, Saccone N, Foroud T, Nurnberger J, Jr., Xuei X, Conneally PM, Schuckit M, Almasy L, Crowe R, Kuperman S, Kramer J, Tischfield JA, Hesselbrock V, Edenberg HJ, Porjesz B, Rice JP, Bierut L and Goate A. in press. A Systematic SNP Screen to Fine-Map Alcohol Dependence Genes on Chromosome 7 Identifies Association with a Novel Susceptibility Gene ACN9, Biological Psychiatry.

Dick DM, Bierut L., Hinrichs A, Fox L, Bucholz KK, Kramer J, Kuperman S, Hesselbrock V, Schuckit M, Almasy L, Tischfield J, Porjesz B, Begleiter H, Nurnberger Jr, J, Xuei X, Edenberg HJ, and Foroud T. 2006. The role of GABRA2 in risk for conduct disorder and alcohol and drug dependence across developmental stages. Behavior Genetics 36(4):577-590.

Dick DM, Plunkett J, Wetherill LF, Xuei X, Goate A, Hesselbrock V, Schuckit M, Crowe R, Edenberg HJ, and Foroud T. 2006. Association between GABRA1 and Drinking Behaviors in the Collaborative Study on the Genetics of Alcoholism Sample; Alcoholism: Clinical and Experimental Research 30(7):11011110 . 
Edenberg HJ, Xuei X, Chen H-J, Tian H, Wetherill LF, Dick DM, Almasy L, Bierut L, Bucholz KK, Goate A, Hesselbrock V, Kuperman S, Nurnberger J, Jr, Porjesz B, Rice J, Schuckit M, Tischfield J, Beglieter H, Foroud T. 2006. Association of alcohol dehydrogenase genes with alcohol dependence: A comprehensive analysis. Human Molecular Genetics 15(9):1539-1549.

Jones KA, Begleiter H, Porjesz B, Almasy L, Goate A, Hinrichs T, Kwon J, Rice JP, Rohrbaugh J, Wang JC, Bauer LO, Chorlian DB, Dick DM, Edenberg HJ, Foroud T, Hesselbrock V, Kuperman S, Nurnberger Jr., J, O'Connor SJ, Schuckit MA, Stimus AT, Reich T. 2004. Linkage and linkage disequilibrium of evoked EEG oscillations with CHRM2 receptor gene polymorphisms: Implications for human brain dynamics and cognition. International Journal of Psychophysiology 53:75-90. Wang JC, Hinrichs AL, Stock H, Budde J, Allen R, Bertelsen S, Kwon JM, Wu W, Dick DM, Rice J, Jones K, Nurnberger JI Jr, Tischfield J, Porjesz B, Edenberg HJ, Hesselbrock V, Crowe R, Schuckit M, Begleiter H, Reich T, Goate AM, Bierut LJ. 2004. Evidence of common and specific genetic effects: association of the muscarinic acetylcholine receptor M2 (CHRM2) gene with alcohol dependence and major depressive syndrome. Human Molecular Genetics 13:1903-1911.

\section{FUNCTIONAL CHARACTERIZATION OF GENES/VARIANTS INVOLVED IN NICOTINE DEPENDENCE Reported by Tania DasBanerjee}

This was a symposium comprised of four speakers and aimed at understanding the genetics of nicotine dependence.

The first presentation was Genome wide association and candidate gene study for nicotine dependence given by Laura Bierut. Nicotine dependence is a many step process: first comes initiation, then smoking, and finally, after smoking about 100 cigarettes, nicotine dependence. Since smoking is the leading source of preventable death in the US and twin studies have consistently demonstrated strong genetic contributions to smoking, this study was undertaken. This presentation described the NICSNP project, a hybrid study of genome wide association (GWA) coupled with analysis of candidate gene results.

The study sample consisted of 1050 nicotine dependent cases and 879 non-dependent smokers as controls. All the participants were selected from two communitybased studies, the Collaborative Genetics Study of Nicotine Dependence (US) and the Nicotine Addiction Genetics Project (Australia). Subjects were chosen on the basis of their Fagerström Test for Nicotine Dependence (FTND) scores: nicotine-dependent smokers had to have a FTND score $>4$ (mean FTND score $=6.3$ ) while the controls (non-dependent smokers) had to have a lifetime FTND score $=0$. The GWA study performed pooled genotyping of 2.4 million single nucleotide polymorphisms (SNPs) followed by individual genotyping of top 40,000 signals. In the candidate gene study, individual genotyping was conducted in 300 genes chosen on the basis of their known functional significance.

The GWA study did not identify any significant SNPs (odds ratio 1.2-2.1) although the results did identify novel loci not previously associated with the risk for nicotine dependence. The candidate gene study revealed significant associations of the GABRA4, CHRNB3, CHRNA3 and CHRNA5 genes with nicotine dependence. In the alpha 5 nicotinic receptor (CHRNA5), a common variant which causes an amino acid change (D398N) in the receptor protein, is highly associated with nicotine dependence. The D398N variance was also replicated in the COGA (Collaborative Study on the Genetics of Alcoholism) samples. It was shown that the D398N variance differs in allele frequency. It is only seen in Europeans, highest among the French, and is not seen in Africans and Asians. These findings are a major step towards our understanding of the genetics of nicotine dependence. The genetic data from this study are available for further analyses by others in the scientific community.

The second presentation was Nicotinic receptor polymorphisms and nicotine sensitivity in mice by Jerry Stitzel. A naturally occurring non-synonymous polymorphism (T529A) in the nicotinic receptor alpha4 subunit gene (Chrna4) has been known to be linked to various measures of nicotine sensitivity, nicotinic receptor function and ethanol-induced depression. This study utilized Chrna4 T529A knock-in mice to validate the role of this polymorphism in modulating sensitivity to nicotine and nicotine receptor function.

Adult mice possessing the T529A allele of Chrna4 differ from controls in nicotine-induced hypothermia but not expression of alpha4beta2 Chrna4 in the whole brain. Preliminary data also shows that the T529A polymorphism influences the function of the alpha4beta2 nicotinic receptors in selected brain regions by shifting the receptor conformation from low sensitivity to high sensitivity. The presence of the T529A allele alters sensitivity to reinforcing properties of nicotine. T529A homozygotes developed nicotine conditioned place preference and failed to develop conditioned taste aversion while A529 homozygotes developed conditioned place aversion as well as conditioned taste aversion. Adult mice possessing the T529A allele of Chrna4 consumed more nicotine by choice than their A529 littermates. Since tobacco consumption begins in adolescence, the above experiments were repeated in adolescent mice with the T529A polymorphism and their A529 littermates. Preliminary results show that adolescent mice homozygous for the T529A allele of Chrna4 consume more nicotine by choice than do their A529 homozygous littermates. It was hypothesized that the T529A variant affects Chrna4 receptor function by modulating the phosphorylation of the serine 530 
residue of the receptor. Whether S530 is actually phosphorylated and how T529A modulates S530 phosphorylation is under investigation. Also, whether the T529A polymorphism affects the valence of nicotine in adolescent mice is yet to be addressed.

The third presentation, Nicotine-dependent behaviors and TRP channels in C. Elegans was given by Shawn $\mathrm{Xu}$. Since the underlying genetic mechanism by which nicotine induces profound behavioral changes in mammals is not well understood, this report used the nematode C. elegans as a genetic model to characterize nicotine response. C. elegans is a good system to study since they are genetically $40 \%$ homologous to humans, have a much simpler nervous system and their wiring pattern is completely known. It has been shown that the worms exhibit similar behavioral responses to nicotine as those observed in mammals, including acute response, withdrawal and tolerance. It has also been shown that repeated intermittent nicotine exposure sensitizes these worms response to nicotine, which is important in the developing of nicotine dependence. Thus this system can be used as a model to study genes underlying these behavioral responses.

Nicotine dependence in C. elegans requires the presence of nicotine receptors. C. elegans contains 29 nicotinic acetylcholine receptor (nAChR) genes and mutation in the acr 15 and acr 16 genes is known to reduce nicotine dependence through reduction in sensitization to nicotine. Remarkably, transgenic expression in C. elegans of the mouse nAChR a4b2, which is known to be critical for nicotine dependence in mice, can rescue the defect of C. elegans nAChR mutants in response to nicotine. In an effort to identify any novel protein regulating nicotine responses, it was found that mutant worms lacking a TRPC channel are defective in response to nicotine. This defect could be rescued by a human TRPC channel, suggesting that TRPC channels are functionally conserved in nicotine responses.

TRPC channels are expressed in the command interneuron and are required for nicotine-dependent behavior. A hypothetical model in place suggests that when nicotine acts on $\mathrm{nAChR}$ on dopamine neurons it causes the release of dopamine from these neurons. This dopamine then binds to and activates metabotropic dopamine receptors on command interneuron, which then through PLC and DAG activate the TRPC channels. The calcium transients created by TRPC channels modulate the behavioral response to nicotine.

This study thus revealed an unexpected role for TRPC channels in nicotine-dependent behavior and raises the possibility that these channels might regulate nicotine dependence in mammals.

The fourth and final presentation was on: The cannabinoid receptor 1 (CNR1) gene association with smoking initiation, nicotine dependence and other substance use by Xiangning Chen. The endogenous cannabinoid system has been implicated in drug addiction in animal models. The CNR1 gene is mainly expressed in the central nervous system (CNS) and is known to be associated with substance abuse, schizophrenia and Parkinson's disease. This study investigated the association of CNR1 with smoking initiation (SI), nicotine dependence (ND) and other substance abuse by typing 10 SNPs in two independent samples, VAND and VAANX. Allelic and haplotype associations were performed.

In the VAND sample $(n=688)$, selected for low and high levels of ND, (on the basis of FTND scores), 10 SNPs were typed in the CNR1 gene. In association analyses, two markers (rs6928499 and rs2023239) were significant for both SI and ND and the association was sex specific. In multi-marker haplotype analyses, highly significant haplotype associations were found for marker combination rs6928499-rs2023239-rs12720071rs806388. In the VAANX sample $(n=961)$, selected by anxiety and neuroticism, the same 10 SNPs were typed. The same two SNPs showed the same sex-specific associations with SI, but no association with ND. In haplotype analyses, significant results were obtained for the same 4-marker combination for both SI and ND. Single marker and haplotype associations' analyses were also done for other substance use/abuse (alcohol abuse, stimulant use, sedative use, and cannabis use) and nominally significant results were observed for multiple substances. These associations were also sexspecific.

These results suggest that the CNR1 gene may be associated with SI, ND and substance use/abuse and that these associations may be sex-specific but not substance specific. There is an estrogen response element (ERE) in the CNR1 gene which may account for the sex-specific effects.

\section{GENETIC INFLUENCE ON ADULT NEUROGENESIS Reported by Dylan Glubb}

It has been well established that adult neurogenesis takes place in the olfactory bulb and the dentate gyrus of the hippocampus (Gould, 2007; Kempermann and Gage, 1999; Lledo and Saghatelyan, 2005; Ming and Song, 2005; Schinder and Gage, 2004; Temple and AlvarezBuylla, 1999). Over 9000 new cells are generated every day in the dentate gyrus of a young adult rat (Cameron and McKay, 2001) but these numbers decline with age (Klempin and Kempermann, 2007). Experience also modulates adult neurogenesis. For example, physical exercise enhances adult neurogenesis in the hippocampus (van Praag et al., 1999) whilst motherhood in female rats can inhibit neurogenesis (Leuner et al., 2007). Stress hormone levels are affected by experience and may determine the resulting change in neurogenesis. Extended running in hypertensive rats, which is associated with an increase in plasma corticosterone levels, actually inhibits exercise induced hippocampal neurogenesis (Naylor et al., 2005). Neurogenesis is 
suppressed in lactating postpartum females until weaning but this decrease in cell proliferation is dependent on elevated glucocorticoid levels associated with lactation (Leuner et al., 2007). Parenting may not always have a negative effect on neurogenesis. Dr Gould commented on the observations of enhanced dendritic spine density during fatherhood in male marmosets. Thus, it appears that neurogenesis and structural plasticity are characteristic of the adult mammalian brain and these phenomena are modulated by experience.

There is evidence for adult neurogenesis in brain regions other than the hippocampus and olfactory bulb. Adult neurogenesis has been observed in the neocortex, striatum, amygdala and substantia nigra but some studies have not been able to replicate these findings (Gould, 2007). In the case of neurogenesis in the adult neocortex, Dr Cameron presented evidence to show neurogenesis occurs there at a similar rate to that in the dentate gyrus. These newborn neurons in the neocortex are immunoreactive for GABA/GAD-67 providing evidence that theses cells are GABAergic interneurons (Dayer et al., 2005). Expression of calcium-binding proteins calbindin and calretinin has also been detected and the temporal expression pattern of these proteins may be indicative of the maturation stage of the new neurons (Dayer et al., 2005). Over 90\% of proliferating cells in the neocortex express NG2 which is considered to be a marker for oligodendrocyte precursors. However, not all NG+ cells differentiate into glial cells and some $\mathrm{NG}+$ cells differentiate into neurons. Therefore, Dr Cameron concluded that these $\mathrm{NG}+$ cells generate GABAergic interneurons in the neocortex.

\section{Neurogenesis and the making of memories}

The hippocampus plays an important role in the learning and the consolidation of memory and the survival of newborn neurons in the hippocampus may be associated with this. One type of learning that is dependent on the hippocampus is the trace eyeblink conditioning task in which there is an interval of time between the presentation of the conditioned and unconditioned stimuli. In the adult rat dentate gyrus, the number of new neurons maintained after this hippocampus-dependent task is significantly greater than the number which survive after a hippocampusindependent task (Gould et al., 1999). The question is whether the learning-induced effects of neurogenesis require a temporal gap between stimuli. To answer this question two alternate versions of trace and delay eyeblink conditioning were developed. In the very long delay eyeblink conditioning task, the duration of the conditioning stimulus is nearly doubled which makes the task hippocampus-dependent and the survival of new neurons is increased (Leuner et al., 2006). In the other task, trace eyeblink conditioning is made hippocampus-independent by training the rats with a delay arrangement and there is no enhanced survival of neurons. These findings indicate that associative learning rather than training increases adult neurogenesis, regardless of a temporal gap between stimuli. Indeed, it may be the acquisition of new information which prevents these new neurons from dying (Dalla et al., 2007) and the quality of the learning may relate to the number of neurons which survive (Sisti et al., 2007). Egr1 and learning

Egr1 is an immediate early gene which is upregulated during associative learning and long term potentiation (Davis et al., 2003). Expression of this gene may be related to neuronal activation (Jessberger and Kempermann, 2003) and Egr1 is also up-regulated following behavioral training using the Morris water maze (Pollak et al., 2005). Other evidence also shows that this gene may play a critical role in learning and memory (Jones et al., 2001). mRNA expression of Egr 1 in the hippocampus is greater in animals which undergo long varied training in the Morris water maze compared with those which receive unvaried training. However, cage controls have higher expression of Egr1 than both groups. Dr Cameron suggested that this effect in the control animals may be due to sleep-based consolidation of memories or related to the lack of stress/low corticosteroid levels that the animals experienced. There also appears to be an age dependence of Egr1 expression in newborn neurons. A maturation period of several weeks is needed before new neurons in the hippocampus will express Egr1 after neuronal stimulation by kainate (Jessberger and Kempermann, 2003).

Akt kinase signaling in learning and synaptic plasticity Akt is a serine/threonine kinase which regulates neuronal cell survival, proliferation and differentiation (Peltier et al., 2007; Zhao et al., 2006), and neuronal excitability (Chae et al., 2005). This kinase has lower activity in the prefrontal cortex of depressed suicide victims (Karege et al., 2007) and is dysregulated in neurodegeneration (Bi et al., 2005; Colin et al., 2005). Activation of Akt may play a role in synaptic plasticity and the consolidation of memory (Horwood et al., 2006). Dr Franke discussed experiments in which Akt1 $1^{(-)}$ and Akt-mutant transgenic mice showed impaired learning in passive/avoidance tasks. Both types of mice also have decreased long term potentiation in the hippocampus. The NMDA NR1 subunit is involved in memory formation (Cheli et al., 2006) and is phosphorylated after training in mice. However, NMDA NR1 phosphorylation is not observed in Akt1 ${ }^{(-/-)}$animals. Therefore, Dr Franke concluded that Akt signaling is important in learning and higher brain function.

\section{References}

Bi X, Liu J, Yao Y, Baudry M, and Lynch G. 2005. Deregulation of the phosphatidylinositol-3 kinase signaling cascade is associated with neurodegeneration 
in Npc1-/- mouse brain. Am J Pathol 167:10811092.

Cameron HA, and McKay RD. 2001. Adult neurogenesis produces a large pool of new granule cells in the dentate gyrus. J Comp Neurol 435:406-417.

Chae KS, Martin-Caraballo M, Anderson M, and Dryer SE. 2005. Akt activation is necessary for growth factorinduced trafficking of functional $\mathrm{K}(\mathrm{Ca})$ channels in developing parasympathetic neurons. J Neurophysiol 93:1174-1182. Epub 2004 Oct 27.

Cheli V, Adrover M, Blanco C, Ferrari C, Cornea A, Pitossi F, Epstein AL, and Jerusalinsky D. 2006. Knocking-down the NMDAR1 subunit in a limited amount of neurons in the rat hippocampus impairs learning. J Neurochem 97:68-73.

Colin E, Regulier E, Perrin V, Durr A, Brice A, Aebischer P, Deglon N, Humbert S, and Saudou F. 2005. Akt is altered in an animal model of Huntington's disease and in patients. Eur J Neurosci 21:1478-1488.

Dalla C, Bangasser DA, Edgecomb C, and Shors TJ. 2007. Neurogenesis and learning: acquisition and asymptotic performance predict how many new cells survive in the hippocampus. Neurobiol Learn Mem 88:143-148. Epub 2007 Apr 6.

Davis S, Bozon B, and Laroche S. 2003. How necessary is the activation of the immediate early gene zif268 in synaptic plasticity and learning? Behav Brain Res 142:17-30.

Dayer AG, Cleaver KM, Abouantoun T, and Cameron HA. 2005. New GABAergic interneurons in the adult neocortex and striatum are generated from different precursors. J Cell Biol 168:415-427.

Gould E. 2007. How widespread is adult neurogenesis in mammals? Nat Rev Neurosci 8:481-488.

Gould E, Beylin A, Tanapat P, Reeves A, and Shors TJ. 1999. Learning enhances adult neurogenesis in the hippocampal formation. Nat Neurosci 2:260-265.

Horwood JM, Dufour F, Laroche S, and Davis S. 2006. Signalling mechanisms mediated by the phosphoinositide 3-kinase/Akt cascade in synaptic plasticity and memory in the rat. Eur J Neurosci 23:33753384 .

Jessberger S, and Kempermann G. 2003. Adult-born hippocampal neurons mature into activity-dependent responsiveness. Eur J Neurosci 18:2707-2712.

Jones MW, Errington ML, French PJ, Fine A, Bliss TV, Garel S, Charnay P, Bozon B, Laroche, S, and Davis S. 2001. A requirement for the immediate early gene Zif268 in the expression of late LTP and long-term memories. Nat Neurosci 4:289-296.

Karege F, Perroud N, Burkhardt S, Schwald M, Ballmann E, La Harpe R, and Malafosse A. 2007. Alteration in kinase activity but not in protein levels of protein kinase B and glycogen synthase kinase-3beta in ventral prefrontal cortex of depressed suicide victims. Biol Psychiatry 61:240-245. Epub 2006 Jul 28.

Kempermann G, and Gage FH. 1999. New nerve cells for the adult brain. Sci Am 280:48-53.
Klempin F, and Kempermann G. 2007. Adult hippocampal neurogenesis and aging. Eur Arch Psychiatry Clin Neurosci 257:271-280.

Leuner B, Mirescu C, Noiman L, Gould E. 2007. Maternal experience inhibits the production of immature neurons in the hippocampus during the postpartum period through elevations in adrenal steroids. Hippocampus 17:434-442.

Leuner B, Waddell J, Gould E, Shors TJ. 2006. Temporal discontiguity is neither necessary nor sufficient for learning-induced effects on adult neurogenesis. $J$ Neurosci 26:13437-13442.

Lledo PM, and Saghatelyan A. 2005. Integrating new neurons into the adult olfactory bulb: joining the network, life-death decisions, and the effects of sensory experience. Trends Neurosci 28:248-54.

Ming GL, and Song H. 2005. Adult neurogenesis in the mammalian central nervous system. Annu Rev Neurosci 28:223-250.

Naylor AS, Persson AI, Eriksson PS, Jonsdottir IH, and Thorlin T. 2005. Extended voluntary running inhibits exercise-induced adult hippocampal progenitor proliferation in the spontaneously hypertensive rat. $J$ Neurophysiol 93:2406-2414. Epub 2004 Dec 22.

Peltier J, O'Neill A, and Schaffer DV. 2007. PI3K/Akt and CREB regulate adult neural hippocampal progenitor proliferation and differentiation. Dev Neurobiol 67:1348-1361.

Pollak DD, Herkner K, Hoeger H, and Lubec G. 2005. Behavioral testing upregulates pCaMKII, BDNF, PSD95 and egr-1 in hippocampus of FVB/N mice. Behav Brain Res 163:128-135.

Schinder AF, and Gage FH. 2004. A hypothesis about the role of adult neurogenesis in hippocampal function. Physiology (Bethesda) 19:253-261.

Sisti HM, Glass AL, and Shors TJ. 2007. Neurogenesis and the spacing effect: learning over time enhances memory and the survival of new neurons. Learn Mem 14:368-375. Print 2007.

Temple S, and Alvarez-Buylla A. 1999. Stem cells in the adult mammalian central nervous system. Curr Opin Neurobiol 9:135-141.

Van Praag H, Kempermann G, and Gage FH. 1999. Running increases cell proliferation and neurogenesis in the adult mouse dentate gyrus. Nat Neurosci 2:266270 .

Zhao H, Sapolsky RM, and Steinberg GK. 2006. Phosphoinositide-3-kinase/akt survival signal pathways are implicated in neuronal survival after stroke. Mol Neurobiol 34:249-270.

\section{PSYCHIATRIC GENETICS AND PHARMACOTHERAPY Reported by Eric J. Vallender}

One of the promises of psychiatric genetics is for the development of efficacious treatments for disease. While much of the efforts in the field still revolve around 
identification of the genes and genetic networks underlying psychiatric disease, an emerging field is focused on the genetic variation underlying the differential responses to treatment of disease. This has focused primarily on varying efficacy rates of pharmacotherapies, but can be extended to other treatment options as well. The focus of the "Psychiatric Genetics and Pharmacotherapy" session was to highlight the work being done in the field and to offer insight into study design and interpretation moving forward.

Schizophrenia: From Gene Arrays to Novel Drug Targets: Dr. Catherine Winchester presented a rodent model of schizophrenia and its uses to develop novel drugs for treatment. The model, which uses rats treated with chronic intermittent phencyclidine (PCP), mirrors the cognitive deficits that are a hallmark of schizophrenia [Morris et al., 2005]. Specifically, schizophrenic patients tend to present with deficits in attention, cognitive flexibility, and goal setting behaviors. These behaviors are correlated with prefrontal cortex hypofunction and can be behaviorally modeled in the rodents [Cochran et al., 2003].

Using RNA isolated from the prefrontal cortex of the rats, the transcriptome of the schizophrenia model was interrogated. The results were encouraging with genes and pathways previously implicated in human schizophrenia showing differential expression and with $20 \%$ of the differentially regulated transcripts in rats mapping to syntenic regions previously showing an association in humans. Additional novel pathways were identified that may serve as potential drug targets and collaborations are underway to develop these opportunities. This will be additionally aided through the development of a gene array focused specifically on genes implicated in the schizophrenic phenotype. Initial studies using clozapine, an antipsychotic commonly used in the treatment of schizophrenia, failed to show a reversal in the transcriptome of the rats. This parallels observations in patients regarding treatment efficacy related to cognitive aspects of the disease.

During discussion several other uses of the data were approached. It was stressed that the rat PCP model focuses on the cognitive deficits associated with schizophrenia and that many of the current anti-psychotics, including clozapine, are limited in their efficacy in treating this aspect of the disease. At this point other antipsychotics had not been modeled in the system, but the framework was there for future studies. While it was acknowledged that the PCP rat model does not model the dopaminergic perturbations seen in human patients, it was noted that it does reflect the cognitive deficits that are a major aspect of the disease. Another question arose as to the possible use of any of these differentially regulated genes in the prefrontal cortex as early diagnostic indicators for schizophrenia; work which will require future studies. It was also observed that the directionality of expression differences in the rat and human were not always the same, leading the researchers to focus on disruptions in pathways that may represent the disease state rather than focus on individual genes.

Pharmacogenetics of Treatments Outcome and Adverse Effects in the $S T A R^{*} D$ Cohort. Research into the association between genetic variation and treatment effects in the STAR*D cohort of individuals with major depression was presented by Dr. Francis McMahon. The STAR*D cohort of individuals with major depressive disorder (MDD) underwent standard clinical treatment wherein they were treated with the antidepressant selective serotonin reuptake inhibitor (SSRI) citalopram when appropriate. They were then evaluated for remission of symptoms, response to pharmacological intervention, and tolerance of the drug. These individuals were then tested for genotypes at 68 candidate genes chosen for previous association with mood disorder, antidepressant outcome, or known functional variation using a split-sample, test/replication design.

Several positive associations have come from this data including an association between HTR2A and nonresponsiveness to citalopram (McMahon et al., 2006), GRIK4 and both remission and response (Paddock et al., 2007), and BCL2 and response. (Non-responsiveness was chosen as the most appropriate measure for comparison of treatment in order to exclude patients showing a placebo effect.) These genes also showed interactions which resulted in lessened non-responsiveness to treatment. Additionally, the cohort was able to replicate findings of an association between FKBP 5 and remission rates.

A further study showed an association between the serotonin transporter promoter polymorphism (5HTTLPR) and tolerability but not treatment outcome (Hu et al., 2007). This is noteworthy for several reasons. First, the effect was seen between the high expression $\mathrm{L}_{\mathrm{A}}$ allele (associated with greater tolerance) compared to the low expression $\mathrm{S}$ and $\mathrm{L}_{\mathrm{G}}$ alleles (associated with lesser tolerance); a dichotomous S vs. L comparison failed to identify association. Secondly, the effect was associated with tolerability rather than response. It is possible that differences seen in previous studies attempting to associate 5HTTLPR with antidepressant response are a result of either the lack of differentiation between L alleles or differences in the way drugintolerant patients are treated in the data analysis.

Studies were also undertaken to look for genetic associations with emergent suicidal ideation during anti-depressant treatment. These studies found an association with GRIA3 and GRIK2 in the STAR*D cohort (Laje et al., 2007). A follow-up whole genome association study also identified IL28R and PAPLN as potentially associated with treatment emergent suicidal thinking.

During discussion it was noted that while promising, these pharmacogenetic studies will require greater 
numbers of markers to be fully informative in the future. It was also observed that compliance was not strictly measured in this cohort, for instance by pill counting or through drug levels in the blood, though patients were asked about adherence to medication. Non-compliance was, and is, a potential confound for many pharmacogenetic studies. A final comment noted the overlap between the genes associated with suicidal ideation developing from treatment and those associated with bipolar disorder. While the connection remains unclear, there are reasons to hypothesize an overlap between these gene sets.

\section{Genetic Association Data from GENDEP, a Multicentre} European Study

Dr. Katherine Aitchison presented progress on the genetic association analysis of response to antidepressants in the multicentre, European Commission funded, study GENDEP ("Genome-based Therapeutic Drugs for Depression"). This study recruited individuals of white European ancestry with depression diagnosed using a research diagnostic interview. Individuals with certain comorbidities, including bipolar disorder and schizophrenia, were excluded. Subjects were treated with either nortriptyline (a pronoradrenergic antidepressant for which the primary target is the norepinephrine transporter) or escitalopram, the $S$-enantiomer of the SSRI citalopram (for which the primary neuronal target is the serotonin transporter).

Patient response was measured serially post-baseline using three different measures of depression (the HDRS, the MADRS, and the BDI), comprising a total of 48 questions. Factor analysis on this data resulted in three factors: mood and anxiety, cognitive symptoms, and neurovegetative symptoms [Uher et al., 2007]. While both groups treated with nortriptyline and escitalopram showed substantial improvement over the twelve week period of evaluation, preliminary analyses showed a greater improvement in neurovegetative symptoms in the group treated with the pronoradrenergic while the improvement in mood and anxiety was greater in the group treated with the proserotonergic.

Variation in twelve candidate genes was tested for association with treatment response. In particular, 5HTTLPR genotype was associated with response as measured by the mood and anxiety factor for subjects treated with escitalopram, but not with nortriptyline response or other response factors. Homozygote carriers of the low expressing $\mathrm{S}$ allele responded more poorly than those in the $\mathrm{L} / \mathrm{S}$ and $\mathrm{L} / \mathrm{L}$ genotypic groups. A preliminary analysis using the rs25531 SNP further refines the groups.

Future studies in the system will include stratification analysis as well as incorporating data on adverse effects of the drugs and patterns response in terms of time course. In the discussion, continuing analyses including the interaction between 5HTTLPR and life events were also mentioned.

Whole Genome Association Analysis of Treatment Response in Schizophrenia. A whole genome association study of schizophrenia treatment response was presented by Dr. Anil Malhotra. The study began as a whole genome association study for schizophrenia using 178 cases and 144 controls. All individuals were recruited and evaluated at a single site and were matched for ethnicity, general environment, and socioeconomic background. This study identified an association between the cytokine-related genes CSF2RA and IL3RA and schizophrenia spectrum disorders [Lencz et al., 2007]. Resequencing studies subsequently showed both common intronic haplotypes as well as rare missense variations associating with the disease.

Patients were clinically assessed and treated either using the antipsychotic clozapine or using a nonclozapine based treatment strategy. Traditionally clozapine has been reserved for patients who fail to show a response to prior treatment; as such the clozapine treated group was used as a proxy for non-responsiveness to schizophrenia medications while the nonclozapine treated group proxied responsiveness. A whole genome association study comparing the 62 nonresponsive patients to the 110 responsive patients identified SNPs in 5 genes differentially distributed between the groups. Several of these genes had a "biological plausibility" for a difference in treatment efficacy of schizophrenia.

The importance of first episode studies was highlighted during discussion. In particular it was relevant to understand prior treatment histories and the effects that may have on the pharmacogenetic association studies. Similarly the importance of a priori assigned response criteria was stressed. The findings presented, however, offered a first look at the methods that can hope to be employed and yielded promising initial results. Future studies on the response of first episode schizophrenia subjects to additional antipsychotics will incorporate these findings.

Alcohol Dependence Associated SNPs in Genes Encoding GABA-A Subunits Predict Post-Treatment Drinking Behavior in Project MATCH. Genetic variation has also been shown to influences the effectiveness of psychosocial treatment. Dr. Jonathan Covault presented on the effects of genetic polymorphism in the efficacy of treatment of alcoholism in Project MATCH. Project MATCH is a multi-center clinical trial of Cognitive Behavioral Therapy (CBT), Motivational Enhancement Therapy (MET) and Twelve Step Facilitation (TSF) as treatment therapies for alcoholism. Levels of drinking were measured during the treatment period (12 weeks) as well as during post-treatment (12 months). During the original study, DNA was collected, but has remained unanalyzed until now. 
Initial genetic association studies have focused on the GABA-A receptors. The Collaborative Study on the Genetics of Alcoholism (COGA) initially identified an association between GABRA2 and alcoholic behavior that was later widely replicated [Edenberg et al. 2004]. In the Project MATCH cohort there was also association seen with alcohol dependence and GABRG1, located immediately telomeric of GABRA2 on $4 \mathrm{p}$ (Covault et al., 2007). Haplotypes of GABRA2 and GABRG1 were in partial linkage disequilibrium in the sample resulting in the inability to resolve risk associated with each separately.

Individuals harboring the alleles of GABRA2/ GABRG1 associated with a greater proclivity towards alcohol dependence did not show a significant difference in treatment efficacy among the three psychosocial treatments under study, however individuals with the low-risk alleles at the genes showed variation in treatment response with TSF having more favorable outcomes compared to MET or CBT. This may indicate that those individuals with high-risk alleles are better candidates for pharmacotherapy while those with low-risk alleles are better candidates for psychosocial therapy.

The differences between $G A B R A 2$ and GABRG1 were briefly discussed; specifically that GABRA2 was ubiquitously expressed while GABRG1 had a much more restricted expression pattern specific to neurons. This may have implications for interpreting the association results. Also focus turned to how pharmacotherapy, in particular with benzodiazepines, would affect response to psychosocial treatments. At the time of the study pharmacotherapy was excluded, but it is a reasonable hypothesis that concurrent treatment with pharmacological agents would affect the differences seen in psychosocial therapy efficacies.

Pharmacogenetics of Antidepressants in Mood Disorder: A Proposal for Methodological Guidelines. Dr. Alessandro Serretti concluded the session with a discourse on the methodological procedures and controls needed to ensure high quality, meaningful results in studies of mood disorder pharmacogenetics. As pharmacogenetic studies of mood disorders have proliferated so have differences in methodologies and heterogeneities in approaches and samples. A summary of the issues and variables surrounding these studies was presented to compliment published work (Serretti et al., 2007).

Numerous methodological issues requiring attention in the literature were raised generally falling into the categories of socio-demographic differences, clinical, treatment, and assessment issues, and statistical and genetic issues. Socio-demographic, statistical, and genetic issues transcend pharmacogenetic studies and have been shown to be important in numerous association studies. Control for age, sex, ethnicity, or socioeconomic status should be de rigueur for association studies, but the difficulty in obtaining the number of patients required to gain power may have lead to compromises here. Genetically, it is necessary to account for all genetic variants and for partial and unspecific effects. Statistically it is important that power analyses be employed, that the same population not be employed for repeated analyses, and that negative findings are reported to properly correct for multiple testing biases. Equally important is for the statistical methods used to accurately reflect the underlying model, with both simple univariate analyses required as well as more complex multivariate and non-parametric models.

Issues in clinical, treatment, and assessment methodologies are more specific to psychiatric studies in general and pharmacogenetic studies in particular. Patients with comorbid disorders need to be excluded and severity and persistence of symptoms made clear. The base clinical indications also need to be made clear for future comparisons to be valid. It is of particular importance in pharmacogenetic studies that patients be first episode or drug naïve, and when not that this be taken into consideration in analysis as number and length of episodes may strongly affect outcome. During treatment it is also important that compliance be closely monitored and that concomitant drugs be avoided or controlled for (including a washout period before commencement of the study). When comparing drugs, it is also important for differences in mechanisms of action to be considered. Assessment similarly must be standardized, including studies of side effects, with both frequent early assessments as well as long term studies.

Unsurprisingly, the discussion focused on how these proposals could be implemented practically. Questions were raised as to the numbers of individuals necessary to achieve power given the large number of variables and the limitations and assets of existing studies. Certainly many subjects will be needed, but it will vary depending on the study. In the meantime, it will be important to minimize heterogeneity wherever possible and to include as much detail as possible in areas where variation among subjects cannot be avoided. It was also noted that there are publication difficulties as regards to introducing new statistical methodologies and publishing negative results. While these deficits certainly exist, they need to be challenged by the community. In particular, while homogeneity across studies is important, if there are deficits that can be addressed they should be. Current studies simply cannot address all of the concerns raised but as the field progresses controlling for these factors will gain in importance.

\section{References}

Cochran SM, Kennedy M, McKerchar CE, Steward LJ, Pratt JA, Morris BJ. 2003. Induction of metabolic hypofunction and neurochemical deficits after chronic intermittent exposure to phencyclidine: differential modulation by antipsychotic drugs. Neuropsychopharmacology 28(2):265-275. 
Covault J, Gelernter J, Jensen K, Anton R, Kranzler HR. 2007. Markers in the $5^{\prime}$-Region of GABRG1 Associate to Alcohol Dependence and are in Linkage Disequilibrium with Markers in the Adjacent GABRA2 Gene. Neuropsychopharmacology.

Edenberg HJ, Dick DM, Xuei X, Tian H, Almasy L, Bauer LO, Crowe RR, Goate A, Hesselbrock V, Jones K and others. 2004. Variations in GABRA2, encoding the alpha 2 subunit of the GABA(A) receptor, are associated with alcohol dependence and with brain oscillations. Am $J$ Hum Genet 74(4):705-714.

Hu XZ, Rush AJ, Charney D, Wilson AF, Sorant AJ, Papanicolaou GJ, Fava M, Trivedi MH, Wisniewski SR, Laje G and others. 2007. Association between a functional serotonin transporter promoter polymorphism and citalopram treatment in adult outpatients with major depression. Arch Gen Psychiatry 64(7):783-792. Laje G, Paddock S, Manji H, Rush AJ, Wilson AF, Charney D, McMahon FJ. 2007. Genetic markers of suicidal ideation emerging during citalopram treatment of major depression. Am J Psychiatry 164(10):15301538.

Lencz T, Morgan TV, Athanasiou M, Dain B, Reed CR, Kane JM, Kucherlapati R, Malhotra AK. 2007. Converging evidence for a pseudoautosomal cytokine receptor gene locus in schizophrenia. Mol Psychiatry 12(6):572580.

McMahon FJ, Buervenich S, Charney D, Lipsky R, Rush AJ, Wilson AF, Sorant AJ, Papanicolaou GJ, Laje G, Fava $\mathrm{M}$ and others. 2006. Variation in the gene encoding the serotonin $2 \mathrm{~A}$ receptor is associated with outcome of antidepressant treatment. Am J Hum Genet 78(5): 804814.

Morris BJ, Cochran SM, Pratt JA. 2005. PCP: from pharmacology to modelling schizophrenia. Curr Opin Pharmacol 5(1):101-106.

Paddock S, Laje G, Charney D, Rush AJ, Wilson AF, Sorant AJ, Lipsky R, Wisniewski SR, Manji H, McMahon FJ. 2007. Association of GRIK4 with outcome of antidepressant treatment in the STAR*D cohort. Am J Psychiatry 164(8): 1181-1188.

Serretti A, Kato M, Kennedy JL. 2007. Pharmacogenetic studies in depression: a proposal for methodologic guidelines. Pharmacogenomics $J$, in press.

Uher R, Farmer A, Maier W, Rietschel M, Hauser J, Marusic A, Mors O, Elkin A, Williamson RJ, Schmael C and others. 2007. Measuring depression: comparison and integration of three scales in the GENDEP study. Psychol Med 1-12.

\section{NEW BIOINFORMATIC AND COMPUTATIONAL METHODS Reported by Robert Karlsson}

This session consisted of nine short presentations of new tools in bioinformatics and statistical analysis. Each speaker also had a poster at the conference, and was available in a poster viewing session following immedi- ately after the presentation, so interested audience members could find out more about the methods than the 10-minute presentations allowed for in an informal setting. The session was divided into three categories of methods, the first of which was called "Tools for genomewide association studies".

The first speaker in this part of the session was Katherine Todd-Brown, presenting the tools PLINK (by Shaun Purcell), gPLINK, and Haploview for managing, analyzing, and visualizing whole genome association study data. PLINK is the major analysis and data management component, and comes with an evergrowing array of statistical methods. gPLINK is a graphical user interface to PLINK, facilitating the usage of PLINK by giving menu choices for the available actions and analyses, and then sending the user's intent to PLINK (which can run on a different computer or even a compute cluster) for execution. Haploview is a tool that integrates with gPLINK and provides visualization of linkage disequilibrium structure in association samples, as well as several analysis methods. The software packages are open source (GPL), runs on most major platforms (Windows, Mac, Linux), and are freely available from http://pngu.mgh.harvard.edu/purcell/ plink (PLINK, gPLINK) and http://www.broad. mit.edu/mpg/haploview (Haploview).

Next was Manuel Ferreira, presenting two new algorithms for analyzing gene-gene and SNP-SNP interactions in whole genome association study data. The methods have been tested on the STEP-BD and WTCCC datasets for bipolar disorder. No interactions remained significant after correcting for multiple testing, but according to the authors a few interesting findings were made nonetheless, considering the genomic location of the top-ranked SNPs. Both algorithms are included in the PLINK software package.

Final speaker in this section was Lauren Weiss, presenting a new program called COPPER, used for analysis of copy number polymorphisms in GWAS data. The method has been tested on autism and bipolar datasets and identified several copy number polymorphisms with a possible influence on disease susceptibility. Validation of these findings is currently ongoing. COPPER does not yet have a public website, so for more information about this method contact Lauren at laweiss@chgr.mgh.harvard.edu.

The next category of methods was called "Bioinformatic tools". First was Patrick Sullivan, presenting three bioinformatic tools for use in psychiatric genetics: TAMAL, SNP Annotations, and SLEP. TAMAL stands for "Technology And Money Are Limiting", and is used to aid in the selection of representative SNPs for a set of candidate genes. SLEP (Sullivan Lab Evidence Project) is a search engine for results from genome-wide association and linkage studies in psychiatric genetics. The tools are available online at http://neoref.ils. unc.edu/tamal/ (TAMAL) and https://slep. unc.edu/ evidence/ (SNP annotations and SLEP). 
Next was Michael Galsworthy, presenting the tools MEDSUM and BITOLA. MEDSUM is used to summarize information from Pubmed and create profiles for both authors and other search terms. BITOLA is an automated literature search tool, which looks for links between genes, compounds and diseases in several databases. The aim is to discover interesting and previously not examined hypotheses in the links. The tools are available online at http://webtools.mf.uni-lj.si/.

Lars Bertram presented the SchizophreniaGene database. This is a continuously updated collection of results from association studies in schizophrenia, both original studies and meta-analyses. The database can be reached at http://www.szgene.org/.

The final presentation in the bioinformatics section was by Melvin McInnis, representing the National Center for Integrative Biomedical Informatics (NCBI). The NCIBI aims to facilitate research in complex diseases by making large amounts of information and tools from different sources available to researchers in a consistent framework. The tools provided by the NCIBI are available online at http://www.ncibi.org/.

The third and final category of methods in this session was called "Statistical methods". First speaker here was Jason Moore, presenting the Multifactor Dimensionality Reduction algorithm (MDR). MDR can be used in the search of epistasis effects in genetic association data. The algorithm works by transforming the data in a way that makes epistatic effects detectable by other methods such as naive Bayes and logistic regression in the transformed data. More information about the algorithm and download links can be found at http:// www.epistasis.org/ and http://www.multifactordimensionalityreduction.org/.

Final speaker of the session was Cathryn Lewis, presenting a meta-analysis of genome-wide linkage studies in schizophrenia. Her study used the Genome Search Meta-Analysis (GMSA) algorithm, which divides the genome into bins of equal length and compounds scores from several linkage studies over these bins in order to increase the power to detect linkage. GMSA was applied to 31 genome-wide linkage scans to produce a map of the most interesting regions for linkage in schizophrenia. The tool used in the analysis can be downloaded from http://www.kcl.ac.uk/depsta/memoge/ gsma/.

\section{COPY NUMBER VARIATION: IMPLICATION FOR PSYCHIATRIC DISEASE Reported by Srijan Sen and Sandra Villafuerte}

The following presentations took place:

1) Widespread human structural genomic variation: what have we learned over the past 3 years? Presenter: Charles Lee. Harvard Medical School, Pathology, Boston, USA. Dr. Lee started with a brief overview on the current knowledge on copy number variation (CNV), properties and associations with common diseases.
CNVs are deletions and duplications of DNA segments larger than $1 \mathrm{~kb}$ and up to several $\mathrm{Mb}$ in size that are present in variable copy number. In 2004, two studies reported the wide-spread presence of CNVs in healthy individuals (Iafrate et al., 2004; Sebat et al., 2004). He referred to a recent publication (Redon et al., 2006) and online databases that provide a catalog for CNVs: $12 \%$ of the human genome represent discrete $\mathrm{CNV}$ regions (CNVRs) and there are about $70 \mathrm{CNV}$ s per individual. A large percentage of CNVs are localized both in genes that encode products that interact with the environment and in genes associated with Mendelian or complex disorders. Currently, 12 association studies have been reported between CNVs and common diseases. The Structural Genomic Variation project is screening 40 subjects for CNVs at a high resolution of $500 \mathrm{bp}$ in order to provide to the scientific community with absolute CNVs for association studies. 8000 CNVs from healthy individuals are currently listed in the Database of Genomic Variants (http://projects.tcag.ca/variation). Only a fraction have been independently verified and the frequency of certain CNVs can vary among ethnic populations. For clinical phenotypes it is important to map, characterize and catalog all CNVs and compared them to few reference genomes to obtain absolute copy number information. The size, the number and type of genes that lie within CNVs are clinically important. Additional considerations include the characterization of CNVs in other species to explore the possibility of specific sequence motifs present in CNVs. Across primates there is homology of $60-70 \%$. CNVs are generally stable over few generations. CNVs can affect gene expression levels by either altering the actual dosage of a particular gene or indirectly affect regulatory elements. The impact of CNVs on clinical diagnosis may be clear for some CNVs while for others further assessment may be necessary to clarify their clinical relevance. About $20 \%$ of CNVRs identified so far are not covered by the newest generation of genome-wide arrays such as Affymetrix or Illumina probes.

2) Recurrent Genome Structural Variation and Cognitive deficits. Presenter: Evan Eichler. University of Washington and HHMI, Genome Sciences, Seattle, USA. Dr. Eichler described a study aiming to characterize duplicated/deleted regions associated with mental retardation (MR) and developmental delay. Duplicated sequences account for $4-5 \%$ of the genome and can "trick" normal meiotic process resulting in altered copy number variation. Several duplication syndromes include some kind of mental retardation. The study focus on 130 genomic regions as potential candidates for undescribed genomic disorders associated with MR. They compare 530 children with idiopathic MR and other cognitive deficits that present karyotype abnormalities vs. normal controls. A deletion on chromosome $17 q$ was present in four MR individuals but not in 1000 controls. To date, 21 deletions have been found in MR children. The Decode group found that this region is 
inverted in $70 \%$ of Europeans, increased fertility and is under positive selection. All children with the deletion have a parent with the selected inversion, so the allele resulting in increased fecundity has the downside of predisposing to carry the deletion onto the next generation.

3) A large-scale study of de Novo copy number variation in Autism. Presenter: Jonathan Sebat. Cold Spring Harbor Laboratory, Cold Spring Harbor, USA. Dr. Sebat started with a rationale on the implication of CNVs in Autism. Various studies have implicated structural variants across the genome in Autism. Microscopic abnormalities have been identified in 5-7\% of autistic patients. The baseline mutation rate of CNVs is higher than SNPs and confers 100 fold higher risk for disease occurrence. CNVs in Mendelian diseases are rare $(1 / 10,000)$. However, in complex traits, as in Autism, CNVs are present at much higher rate (1/200 subjects if 50 genes are involved in the susceptibility). Comparative genomic hybridization (CGH) was performed on a large sample of families with one (simplex) or multiple (multiplex) offspring with idiopathic autism spectrum disorders in order to detect CNVs in patients and unaffected subjects that are not present in their parents, searching for sporadic or de novo CNVs. A deleted region of 30 genes, including oxytocin and vasopressin, was identified in a simplex patient diagnosed with Asperger syndrome. Between 10 and 15 de novo CNVs have been confirmed in these subjects. All CNVs larger than $4 \mathrm{Mb}$ have been previously identified, while smaller CNVs are de novo. In summary this large scale study has found $10 \%$ of sporadic cases have de novo CNVs ( $25 \%$ of these in female subjects). 9/10 de novo CNVs found in this study are clinically relevant. The presence of de novo CNVs was significantly $(\mathrm{p}=0.0005)$ more frequent in patients $(14 / 195)$ than in controls $(2 / 196)$. The rate of de novo mutations identified in this study is an underestimate, maybe due to the limited resolution of genome microarray scans.

4) CNVs at a bipolar disorder locus on 8q24. Presenter: Margit Burmeister. Molecular \& Behavioral Neuroscience Institute, University of Michigan, Ann Arbor, Michigan, USA. Dr. Burmeister described the detection and analyses of CNVs in a bipolar locus on chromosome 8q24. In an attempt to refine this region, 1536 SNPs were selected for genotyping in 3512 individuals from 734 families with Bipolar Disorder (NIMH, Hopkins) using Illumina Golden Gate. Mendelian errors and deviation from Hardy-Weinberg equilibrium were identified for 14 consecutive SNPs that mapped to a $182 \mathrm{~kb}$ CNV region listed as variation_0337 in the Database of Genomic Variations. The presence or absence of the deletion was tested by PCR, indicating that the deletion was present in carriers from all families with Mendelian errors. To test association between this CNV and Bipolar disorder, an algorithm was developed by a collaborator, Sebastian Zoellner, to use Illumina SNP genotype intensity data to infer carrier status for the deletion/ insertion. This EM algorithm models the intensity distribution for each SNP in the deletion into two groups, heterozygous for the deletion, or absence of the deletion. The algorithm does not call for homozygotes for the deletion. All 3512 individuals were unambiguously scored, 1 individual homozygous for CNV (per PCR), 172 deletion carriers and 3339 not deleted. $4.4 \%$ of carriers for the deletion were among unrelated individuals. The association was tested using the software for Linkage and Association Modeling in Pedigrees (LAMP) [Li et al., 2005 ] to jointly estimate both genotype count in cases and controls and transmission frequency of alleles. The CNV 0337 was not associated with Bipolar disorder in this sample. This algorithm, developed to score presence/absence of known CNVs, will be available shortly as a useful tool to measure CNVs from GoldenGate (and also GWA) SNP chips directly.

\section{Discussion}

The audience expressed interest on the experience these investigators had with the study of CNVs, the impact they may have on psychiatric diseases, considerations for future study designs including methodological issues and relevance of CNVs compared to SNPs in disease or trait susceptibility. Some issues raised in the discussion included:

1. It was suggested that the NIMH has a large group of subjects with psychiatric data which would be good control subjects, but that companies need to create platforms to cover CNVs genotyping.

2 . The need exists for obtaining formal consent to recontact individuals for in depth further phenotyping.

3 . There may be an accumulation of many rare alleles as will be found in CNV studies and these can cause common disease rather than few common variants.

4. A strong linkage signal, does not speak against CNVs as a plausible cause if the CNV is not de novo. It is more likely that a mixed model may explain the signal.

5. The majority of the identified mutations in Autism are not recurrent, but are unique; about 6 are found multiple times. There is a need for large sample size to obtain appropriate characterization of these mutations. 6. It is true that simplex/multiplex families are not clearly distinguishable; but it is important to enrich cohorts for simplex families to increase the identification of de novo variants.

7. If we discard SNPs data that violate Hardy Weinberg Equilibrium, we may be discarding SNPs that are relevant to CNVs. David Altshuler (MIT) developed a program to score SNPs for CNV signatures.

\section{References}

Iafrate AJ, Feuk L, Rivera MN, et al. 2004. Detection of large-scale variation in the human genome. Nat Genet 36:949-951. 
Li M, Boehnke M, Abecasis GR. 2005. Joint modeling of linkage and association: identifying SNPs responsible for a linkage signal. Am J Hum Genet 76:934-949.

Redon R, Ishikawa S, Fitch KR, et al. 2006. Global variation in copy number in the human genome. Nature 444:444-454.

Sebat J, Lakshmi B, Troge J, et al. 2004. Large-scale copy number polymorphism in the human genome. Science 305:525-528.

Sharp AJ, Hansen S, Selzer RR, Cheng Z, Regan R, Hurst JA, Stewart H, Price SM, Blair E, Hennekam RC, Fitzpatrick CA, Segraves R, Richmond TA, Guiver C, Albertson DG, Pinkel D, Eis PS, Schwartz S, Knight SJ, Eichler EE. 2006. Discovery of previously unidentified genomic disorders from the duplication architecture of the human genome. Nat Genet 38(9):1038-1042. Epub 2006 Aug 13. PMID: 16906162.

Sharp AJ, Selzer RR, Veltman JA, Gimelli S, Gimelli G, Striano P, Coppola A, Regan R, Price SM, Knoers NV, Eis PS, Brunner HG, Hennekam RC, Knight SJ, De Vries BB, Zuffardi O, Eichler EE. 2007. Characterization of a recurrent $15 \mathrm{q} 24$ microdeletion syndrome. Hum Mol Genet 16(5):567-572. Epub 2007 Mar 14. PMID: 17360722

\section{GENE EXPRESSION STUDIES Reported by James Crowley}

Modern molecular biological techniques have increased our understanding of the links between gene expression, protein function and behavior. For example, GAD67, RGS4, DTNBP1, NRG1, and GABRAB2 show expression alterations in the postmortem brain of subjects with schizophrenia, and these genes have also been implicated as schizophrenia susceptibility genes (Chen et al., 2004; Volk et al., 2000; Straub et al., 2002; Stefansson et al., 2002; Petryshen et al., 2005; Lo et al., 2004). Gene expression profiling by DNA microarray has proven to be a powerful method to uncover relationships between gene expression alterations and psychiatric disorders. This year's WCPG meeting featured a series of talks by investigators examining the association between disease status and changes in gene expression and protein function.

Dr. Melanie Carless from the Southwest Foundation for Biomedical Research (San Antonio, TX) spoke on "Transcriptional profiling for identification of cis-regulated genes involved in complex brain-related disorders". The genetic determinants of complex brainrelated disorders such as schizophrenia, depression, and Alzheimer's disease remain poorly understood, partly due to an incomplete understanding of normal brain structure and function. Carless and performed genomewide transcriptional profiling in a population of 1,240 individuals in the San Antonio Family Heart Study (SAFHS) using lymphoblast RNA. A total of $\sim 20,000$ transcripts were significantly detected, with 16,829 of these being significantly heritable. There were nearly 3,000 autosomal cis-acting QTLs: linkage evidence for variation at the transcripts genomic location that influences expression levels. Among these cis-regulated transcripts were a number of genes previously implicated in various brain-related mental disorders, including NRG1 (cis-LOD = 15.8), DISC1 (cis$\mathrm{LOD}=3.9)$ and DTNBP1 (cis-LOD $=2.1$ ). They resequenced $2 \mathrm{~kb}$ of the proximal promoter region of these genes in a founder population to identify regulatory variants influencing expression levels, with genotyping efforts expanded to the remaining SAFHS population. To date, they have identified ten polymorphisms (three novel) within the promoter region of NRG1, ten polymorphisms (five novel) within DTNBP1 and nine polymorphisms (three novel) within DISC1.

In sum, significant associations were detected between promoter variants and expression levels of psychiatrically important candidate genes. Identification of functional regulatory variants within these genes will give further insight into how they influence psychiatric disorders.

An audience member asked what the minor allele frequencies (MAFs) were for the proximal promoter region SNPs identified by resequencing. Dr. Carless said that, in general, the previously identified SNPs ranged from 0.3-0.5 MAF, while the novel SNPs were predominantly below $0.05 \mathrm{MAF}$. A second questioner asked if a proteomic analysis would be performed to complement these data. Dr. Carless reported that this will indeed occur and that they have plasma frozen already. The final questioner asked if there were any future plans to look at methylation- Dr. Carless said they were not planning to examine this.

Dr. Herb Lachman from the Albert Einstein College of Medicine (New York, NY) presented "Identification of regulatory domains in schizophrenia and bipolar disorder candidate genes using chromatin immunoprecipitation and tiled microarrays (CHIP-CHIP)". A number of genes have been identified as potential candidates underlying schizophrenia (SZ) and bipolar disorder (BD) susceptibility. However, it has been exceedingly difficult to identify disease associated allelic variants. One reason is that responsible functional alleles may exist in unidentified regulatory domains, which are difficult to characterize. However, in recent years it has become feasible to blindly screen genes in their entirety, including large amounts of flanking DNA, for regulatory domains using microarray-based approaches, such as ChIP-chip. Lachman and colleagues immunoprecipitated chromatin derived from fetal brain using an antibody against histone H3 acetylated at lys9 and lys14 (H3K9/14Ac), a chromatin marker for promoters and some enhancers, and an antibody against histone H3 monomethylated at lys4 (H3K4me1), a marker for enhancers. These were used as probes for DNA microarrays containing the entire sequence of NRG1, DTNBP1, DISC1, including flanking regions, as well as 3 megabases of DNA from the region on $22 q 11$ deleted 
in VCFS. The H3K9/14Ac ChIP-chip experiment clearly identified the promoters active in fetal brain in each of the candidate genes including four found in the complex NRG1 locus. Putative enhancers in several candidate genes were found as well. Dozens of SNPs are known to exist in these regions, some of which will be analyzed as potential disease associated variants. The results indicated that ChIP-chip can be used to map regulatory domains in SZ and BD candidate genes, thereby targeting discrete regions for mutation detection analysis that may identify novel candidate alleles underlying disease susceptibility.

An audience member asked if any adult tissues were examined to determine if the same promoters were active in the human brain. Dr. Lachman replied that they have not yet performed ChIP-chip in adult tissues, but that electrophoretic mobility shift assays (EMSAs) were currently being undertaken in adult tissues.

Dr. Sibylle Schwab from the University of Western Australia (Nedlands, Australia) presented an "Expression study of DTNBP1 in lymphoblastoid cell lines from a family sample with evidence for association". DNA sequence variants (SNPs) located in the dystrobrevin binding protein 1 gene (DTNBP1, dysbindin) gene have been shown to be associated with schizophrenia. However, association is restricted to SNPs in intronic region and no obvious functional SNPs have been identified. Multiple splice transcripts with introns have been identified for dysbindin. Due to the absence of an association with a coding SNP the authors investigated if susceptibility to schizophrenia may be due to altered gene expression of dysbindin. Total RNA extracted from lymphoblast cell-lines of affected individuals was amplified by RT-PCR. Quantitative real time RT-PCR using Taqman probes spanning exon 5/6 and exon 9/10 of DTNBP1 were used to quantify the expression. Relative expression of dysbindin was normalized with two housekeeping genes (GUSB and GAPDH). The DeltaDeltaCt method was used for the analysis of relative expression. Splice variants were compared to those found in RNA from post mortem brain samples. The results indicated that dysbindin was alternatively spliced in lymphoblasts. Using primers located in exon 1 and 6 of the gene, Schwab and colleagues identified a splice variant missing exon 2 and 3. Dysbindin splice variants as detected in RNA from post mortem brain samples revealed the same pattern. In addition, dysbindin mRNA expression was increased in RNA isolated from lymphoblasts from patients with schizophrenia compared to controls. In conclusion, the authors obtained evidence for the existence of dysbindin splice variants in lymphocytes and potential differential regulation of these variants in schizophrenia.

Dr. Schwab was asked whether the increased dysbindin mRNA seen in lymphoblasts from schizophrenis patients translates to increased levels of dysbindin protein. She indicated that her lab is currently performing these experiments.
Dr. James Sutcliffe from Vanderbilt University (Nashville, TN) spoke on "Autism-associated serotonin transporter variants confer gain of function phenotypes arising through distinct mechanisms". The serotonin transporter (SERT) is responsible for 5-HT inactivation in the CNS and peripherally. SERT is implicated in multiple brain disorders, including obsessive-compulsive disorder (OCD), and autism. Sutcliffe and colleagues recently identified multiple autism-associated variants in the SERT gene (SLC6A4), including four rare coding variants (Gly56Ala, Ile425Leu, Phe465Leu and Leu550Val). These variants are associated with more severe OC behaviors in carriers with autism. Other studies found Ile425Val in subjects with Asperger's syndrome and OCD. The authors previously showed that Gly56Ala shows elevated basal activity and insensitivity to $\mathrm{PKG} / \mathrm{p} 38 \mathrm{MAPK}$ regulation. In this study, variant SERTs were examined for altered 5HT uptake, surface and total SERT binding and signaling properties through transfection in HeLa cells. The results indicated that Ile425Leu, Ile425Val, Phe465Leu, and Leu550Val encoded SERT display elevated basal 5HT uptake, resulting from an elevated Vmax. Contrasting Gly56Ala, which shows elevated transport without changes in surface density, the other variants display elevated surface expression that parallels changes in 5HT uptake, suggesting changes in constitutive transporter trafficking. Additionally, Ile425Leu, Ile425Val, Phe465Leu and Leu550Val respond to PKG, p38MAPK and PKC stimulation similar to wild-type SERT.

These studies indicate two distinct mechanisms by which these variants result in increased 5HT transport, suggesting that inappropriately elevated $5 \mathrm{HT}$ clearance and diminished extracellular 5-HT mediated by SERT may be critical risk factors for autism and OCD. In terms of future directions, Dr. Sutcliffe indicated that his group is pursing the hypothesis that loci for other proteins regulating SERT harbor autism-associated risk factors.

Dr. Richard Straub from the National Institutes of Mental Health (Bethesda, MD) discussed "The BLOC-1 protein dysbindin modulates dopamine D2 receptor internalization and signaling but not D1 internalization in rat primary neurons, human neuroblastoma cells, and human B lymphoblasts". Variation in DTNBP1 influences expression, cognition and schizophrenia risk. Expression in schizophrenia is deficient, but the mechanisms by which this contributes to pathogenesis and symptomatology are unknown. One function of dysbindin involves BLOC-1, an endosomal trafficking complex that includes proteins from MUTED and BLOC1S2, genes that the authors have also found to increase risk. Elevated dopamine D2 receptor (DRD2) density has been observed in schizophrenia and DRD2 antagonism appears important to therapeutics, so they studied dysbindin effects on dopamine signaling. The authors transfected siRNA for DTNBP1 or MUTED into rat primary cortical neurons, human SH-SY5Y neuro- 
blastoma cells, and human B lymphoblasts. These cells were then examined for cell surface levels of DRD2 and DRD1 using flow cytometric quantitation, as well as CREB phosphorylation, an intracellular signal downstream of DRD2. They found that DTNBP1 siRNA decreased dysbindin protein, increased DRD2, and completely blocked dopamine-induced DRD2 internalization. MUTED siRNA reduced dysbindin and produced similar effects. DRD1 was unchanged, as were basaland dopamine-induced internalization. Reduction of CREB phosphorylation by the DRD2 agonist quinpirole was enhanced by dysbindin down-regulation. Haloperidol blocked this effect, indicating that the CREB signal required DRD2 stimulation. In conclusion, the authors noted that this was the first demonstration of a functional effect of a schizophrenia susceptibility gene on DRD2 signaling, a pathway long implicated in the illness. They also proposed a molecular mechanism in which DTNBP1 risk alleles down-regulate dysbindin, inhibiting BLOC-1 trafficking of DRD2 towards degradation. Upregulation of DRD2, but not DRD1, contributes to the imbalances in dopaminergic neurotransmission characteristic of schizophrenia. Their observation of statistical epistasis between COMT and DTNBP1 that impairs cognition and increases risk suggests that DRD2 upregulation induced by dysbindin deficiency may be particularly detrimental for COMT Met158Met individuals, due to their elevated cortical dopamine.

An audience member asked whether variation in the dysbindin gene has been associated with risk of tardive dyskinesia, which some suspect results from upregulation of D2 dopamine receptors. Dr. Straub replied that, to his knowledge, this has not been looked at.

In the general panel discussion that followed these presentations, a series of questions related to lymphoblastoid cell lines were raised. The first related to how much variability in gene expression is seen over time in replicates of these cell lines. No member of the panel or audience was aware of a study that had examined this particular question in detail. The second question asked if substantial differences are seen in the gene expression profiles of transformed lymphocytes versus fresh lymphocytes. Again, no member of the panel or audience was aware of a study that had examined this question in detail. A final question was how well the gene expression profile of human lymphoblastoid cell lines relates to that of human brain. A panel member indicated that a study designed to answer this question is currently being performed in baboons, comparing the expression profile of lymphocytes with that of a number of distinct brain regions.

\section{References}

Chen X, Dunham C, Kendler S, Wang X, O'Neill FA, Walsh D and Kendler KS. 2004. Regulator of G-protein signaling 4 (RGS4) gene is associated with schizophre- nia in Irish high density families. Am J Med Genet $B$ Neuropsychiatr Genet 129:23-26.

Volk DW, Austin MC, Pierri JN, Sampson AR and Lewis DA. 2000. Decreased glutamic acid decarboxylase67 messenger RNA expression in a subset of prefrontal cortical gamma-aminobutyric acid neurons in subjects with schizophrenia. Arch Gen Psychiatry 57:237-245.

Straub RE, Jiang Y, MacLean CJ, Ma Y, Webb BT and Myakishev MV et al. 2002. Genetic variation in the $6 \mathrm{p} 22.3$ gene DTNBP1, the human ortholog of the mouse dysbindin gene, is associated with schizophrenia. Am J Hum Genet 71:337-348.

Stefansson H, Sigurdsson E, Steinthorsdottir V, Bjornsdottir S, Sigmundsson T and Ghosh S et al. 2002. Neuregulin 1 and susceptibility to schizophrenia. Am $J$ Hum Genet 71:877-892.

Petryshen TL, Middleton FA, Kirby A, Aldinger KA, Purcell S and Tahl AR et al. 2005. Support for involvement of neuregulin 1 in schizophrenia pathophysiology. Mol Psychiatry 10:366-374.

Lo WS, Lau CF, Xuan Z, Chan CF, Feng GY and He L et al. 2004. Association of SNPs and haplotypes in GABAA receptor beta2 gene with schizophrenia. Mol Psychiatry 9:603-608.

\section{SEX CHROMOSOME ANEUPLOIDIES AND THE SEX CHROMOSOMES IN PSYCHIATRIC DISORDERS Reported by Neetha N. Vijayan}

The World Congress on Psychiatric Genetics 2007, held at New York in October, had a very interesting and stimulating session on sex chromosomes and their influence in psychiatric disorders. This enlightening session was chaired by T.J.Crow from Oxford University and had Lynn Delisi from New York University School of Medicine as discussion moderator, with five presenters in total.

David Skuse from The Institute of Child Health, UK, presented his observations on the impact of human X linked genes on cognition. In addition, experimental data on eye tracking in X-monosomic females demonstrated similar results as in males with Asperger syndrome, implicating the involvement of $\mathrm{X}$ linked genes in the functional integrity of human brain.

An observation on fMRI studies with Klinefelter men was presented by Rene' Kahn, from Rudolf Magnus Institute for Neuroscience, Netherlands. fMRI was used to explore the effects of an extra X chromosome on language lateralization. The data presented clearly suggests that there is decreased language lateralization in Klinefelter men due to increased activity in the language areas of the right hemisphere. This was interesting since loss of language lateralization was also exhibited by patients suffering from schizophrenia.

Another study on Klinefelter men using structural MRI was presented by Jay Geidd, from NIMH. The work highlighted the key difference in structural brain MRI 
imaging of Klinefelter men when compared to healthy age matched controls. Total cerebral volume and lobar volumes were smaller in Klinefelter men and lateralventricle volume was larger. The cortex was thinner in the XXY group in left frontal, temporal, and superior motor regions. These findings are consistent with corresponding cognitive and behavioral problems in Klinefelter men.

Jean Steyaert from Katholieke Universiteit Leuven, Belgium reported results from a study on XYY syndrome in children and its clinical implications. A study on boys with both prenatal and postnatal diagnoses of XYY revealed that they have an increased prevalence of early language problems and increased trend for autism spectrum disorders. This data raises issues about the direct involvement of the $\mathrm{Y}$ chromosome in the social communicative domain.

Finally, Timothy Crow from Oxford University presented his thoughts on the involvement of genes within the X-Y homologous region and hominid evolution. $\mathrm{He}$ concluded that the data from XXY and XYY males were consistent with involvement of an excess of an X-Y homologous gene, one that escapes normal X-chromosome inactivation and is involved with lateralization of the brain and language functioning. The human brain lateralized at some point in evolution and a torque from right frontal to left occipital was introduced. The torque, which is the defining characteristic of human brain is stable across populations, but is epigenetically variable within populations. Normal torque is diminished or absent in schizophrenia. This hypothesis proposes that schizophrenia is a disorder of the genetic mechanisms that controls the mechanism of cerebral asymmetry. Psychosis represents an extreme variation that cuts across populations, relates to cerebral torque, and is associated with the capacity to acquire language. This hypothesis is supported by data from the previous speakers and explains why schizophrenia-like illness is increased among men with XXY, and possibly men with XYY syndrome. On the other hand, non-verbal cognitive deficits and autistic-like behavior are more frequent in females who lack an $\mathrm{X}$ chromosome. He also noted that by assessing the anatomical asymmetry (torque) in Klinefelter's men, a loss of asymmetry anterior but not much posterior was noted. Whereas in Turner's females, an exaggerated occipital asymmetry to the left was noted and this was consistent with its respective symptomatology. Klinefelters have verbal deficits or delays suggesting a left hemisphere dysfunction and Turners have deficits in non verbal (spatial) ability and relative loss of right hemisphere function, consistent with relative torque. Finally he suggested that a brain expressed gene, Protocadherin $\mathrm{X} / \mathrm{Y}$, that has evolved as a uniquely human variant and in an X-Y homologous chromosomal region escaping inactivation was a likely candidate for a gene that contributes to cerebral asymmetry, language and schizophrenia
A discussion following the presentations and was chaired by Lynn Delisi. One question raised was whether it would be important to screen all children with language problems for chromosomal anomalies like Klinefelters. However, Jay Giedd cautioned that these studies reported had enormous bias towards XXY men who had difficulties, and that he has observed individuals without language and other cognitive disabilities who also have an XXY chromosome constitution. He commented that there are likely to be many unrecognized people with an XXY karyotype in the general population.

Another issue raised was that of parental origin of the extra $\mathrm{X}$ chromosome in Klinefelters, triple $\mathrm{X}$ and $\mathrm{X} 0$ and how this might effect the phenotype. David Skuse responded with a summary of some of a recent study he performed involving Klinefelter's subjects who consisted of 50\% paternal-origin to the extra-X and $50 \%$ maternal origin of the extra-X chromosome. Although the sample was small, unlike his previous report in $\mathrm{X} 0$ females, with the XXY males there was no imprinting effect on cognitive deficits.

In summary, the presentations and the discussions in this session highlighted the undeniable influence of sex chromosomes in social cognition, behavior and language development. It seems important to focus future research to unravel the molecular basis behind this phenomenon.

\section{EPIGENETICS Reported by Jonathan Mill}

It is becoming increasingly apparent that there is more to the genome than the series of nucleotides making up our DNA sequence. Sitting above the DNA sequence is a second layer of information (the "epigenome") controlling gene expression and a range of other genomic functions. Epigenetics refers to the mitotically heritable, but reversible, regulation of gene expression mediated principally through modifications of DNA and histones (Henikoff and Matzke, 1997). Such epigenetic processes are essential for normal cellular development and differentiation, and allow the regulation of gene function through non-mutagenic mechanisms. It has been postulated that epigenetic dysregulation could explain the many epidemiological, clinical, and molecular peculiarities associated with psychiatric phenotypes that are hard to rectify using traditional gene- and environment-based approaches (Mill and Petronic, 2007). A dedicated epigenetics plenary symposium at this years' World Congress of Psychiatric Genetics Meeting included talks from four distinguished researchers who introduced some basic epigenetic processes and discussed their potential importance within the context of psychiatric disease. Whilst epigenetic research in psychiatry is in its infancy, and few empirical studies have been performed to date, there was also an interesting poster session, with 
several studies attempting to link DNA methylation changes to psychiatric phenotypes.

The first speaker at the plenary symposium was James Potash from the Center for Epigenetics at Johns Hopkins University School of Medicine, whose talk focused on a study that he and his colleagues have performed investigating DNA Methylation signatures within the human brain. We actually know very little about what comprises a 'normal' epigenome, and how epigenetic marks such as DNA methylation differ between tissues. This is one of the targets of the Human Epigenome Project (www.epigenome.org), which aims to map all methylation variable positions in the genome (Eckhardt et al., 2006). For future etiological studies in psychiatric disorders, it will be important to map brainregion specific epigenetic differences. In research currently 'in press' in the American Journal of Human Genetics, Dr Potash and colleagues investigated 1,505 CpG sites, representing 807 genes, in brain samples from the cerebral cortex, cerebellum, and pons. Analysis of their data showed distinct tissue-specific clustering, and allowed them to identify specific DNA methylation signatures that could distinguish between these three regions.

Mounting evidence suggests that epigenetic processes may be induced following exposure to a range of environmental insults. DNA methylation, for example, has been shown to vary as a function of numerous nutritional, chemical, physical, and psychosocial factors (Feil, 2006). Such dynamic epigenetic changes were the focus of a presentation by Emma Whitelaw from the Queensland Institute of Medical Research in Australia. She discussed the importance of so-called 'metastable epialleles' - loci that can be epigenetically modified to produce a range of phenotypes from genetically identical cells (Rakyan et al., 2002). Many of these loci have been shown to be environmentally regulated; one classic example is the agouti viable yellow allele in mice, in which a promoter-region transposon can be epigenetically altered by manipulating the diet of pregnant females, directly affecting expression of the agouti gene and producing a wide distribution in offspring coat color phenotypes ranging from yellow (unmethylated) to brown (methylated; Cooney et al., 2002). It was traditionally believed that epigenetic profiles are reset and erased during gametogenesis, thus preventing the meiotic transmission of epigenetic information between generations. Dr Whitelaw reviewed mounting evidence to suggest that such notions may be incorrect - i.e. the epigenetic marks of at least some mammalian genes may not be fully erased during meiosis and could be passed transgenerationally. Although still to be proven in humans, her group's studies on animals demonstrate that, at least for some loci, the epigenetic state may be a record of environmental history, including that of previous generations.

Dolores Malaspina, from the Department of Psychiatry at New York University, introduced the idea that aberrant epigenetic regulation could explain the relationship between increasing paternal age and the risk of developing schizophrenia. There are now numerous published replications of her initial data uncovering a three-fold increased risk for offspring of fathers aged over 50 compared to those aged less than 25 years (Malaspina et al., 2001). These cases may account for most sporadic schizophrenia cases, and may comprise a specific subtype of the disease. Given that advancing age and environmental influences have been linked to epigenetic dysfunction, she proposed that epigenetic dysregulation could be the mechanism behind the effects of paternal age. Her group is currently involved in some intriguing animal studies that have found quite distinct phenotypic changes in inbred mice differing only in paternal age. Interestingly, the offspring of these animals reverted to wild-type behaviors, suggesting that these phenotypic effects were not caused by DNA sequence mutations.

The final talk was by Carmen Sapienza from Temple University School of Medicine in Philadelphia, who discussed the importance of interindividual epigenetic variation. His group has used longitudinal studies to track changes in X-chromosome inactivation and genomic imprinting over the life-course, and found evidence that both these become increasingly unstable with age. Of particular interest to psychiatric phenotypes, Dr Sapienza's team has found evidence that DNA methylation in the MAOA gene is highly variable between individuals. He proposed that determining the epigenotype of a gene could provide additional information to that obtained from looking at simple DNA-sequence based changes.

A number of interesting epigenetic posters were also presented at this year's WCPG. Following previous reports suggesting that epigenetic changes in the reelin gene may be associated with schizophrenia (Grayson et al., 2005), Mamoru Tochigi, Tadafumi Kato, and colleagues from the University of Tokyo attempted to replicate this finding using fully quantitative methylation profiling methods but were unable to detect any schizophrenia-associated changes. Optimal methodological approaches for DNA methylation studies are still being developed, and the Japan study highlights how some of the methods used in previous studies, for example methylation-specific PCR, could potentially lead to the biased assessment of methylated cytosines. Dirk Moser, Jobst Meyer, and colleagues from the University of Trier in Germany presented evidence suggesting that a transcription-factor binding site in the glucocorticoid receptor gene exon 1-F promoter, which is epigenetically altered by maternal care in rat pups (Weaver et al., 2004), is not methylated in the human hippocampus, replicating previous analyses of this region performed on lymphocytes and cerebellum tissue (Mill et al., 2005). Jonathan Mill, Arturas Petronis and colleagues from the Centre for Addiction and Mental Health in Toronto presented findings from 
the first comprehensive microarray-based epigenomic study of major psychosis using DNA obtained from the frontal cortex. They reported evidence for psychosisassociated DNA methylation differences in numerous loci, including several involved in glutamatergic and GABAergic neurotransmission, brain development, and other processes functionally-linked to disease etiology. Finally, Go Kuratomi, Tadafumi Kato, and colleagues from RIKEN presented data from their investigation of a pair of monozygotic twins discordant for bipolar disorder. They found evidence for decreased DNA methylation upstream of the gene PPIEL, and a strong inverse correlation between gene expression and methylation levels.

\section{References}

Cooney CA, Dave AA and Wolff GL. 2002. Maternal methyl supplements in mice affect epigenetic variation and DNA methylation of offspring. J Nutr 132:2393S2400S.

Eckhardt F, Lewin J, Cortese R, Rakyan VK, Attwood J, Burger M, Burton J, Cox TV, Davies R, Down TA, Haefliger C, Horton R, Howe K, Jackson DK, Kunde J, Koenig C, Liddle J, Niblett D, Otto T, Pettett R, Seemann S, Thompson C, West T, Rogers J, Olek A, Berlin K, and Beck S. 2006. DNA methylation profiling of human chromosomes 6, 20 and 22. Nat Genet 38:1378-1385.

Feil R. 2006. Environmental and nutritional effects on the epigenetic regulation of genes. Mutat Res 600:46-57. Henikoff S and Matzke MA. 1997. Exploring and explaining epigenetic effects. Trends Genet 13:293295.

Mill J and A Petronis. 2007. Molecular studies of major depressive disorder: the epigenetic perspective. $\mathrm{Mol}$ Psychiatry 12:799-814.

Rakyan VK, Blewitt ME, Druker R, Preis JI, and Whitelaw E. 2002. Metastable epialleles in mammals. Trends Genet 18:348-351.

Malaspina D, Harlap S, Fennig S, Heiman D, Nahon D, Feldman D, and Susser ES. 2001. Advancing paternal age and the risk of schizophrenia. Arch Gen Psychiatry 58:361-367.

Grayson DR, Jia X, Chen Y, Sharma RP, Mitchell CP, Guidotti A, and Costa E. (2005). Reelin promoter hypermethylation in schizophrenia. Proc Natl Acad Sci USA 102:9341-9346.

Mill J, Caspi A, Dempster E, Williams B, Moffitt T, and Craig I. 2005. Methylation analysis of a NGF1-A transcription factor binding-site in the promoter region of the human glucocorticoid receptor gene (NR3C1). American Journal of Medical Genetics Part B: Neuropsychiatric Genetics 138B:89.

Weaver IC, Cervoni N, Champagne FA, D'Alessio AC, Sharma S, Seckl JR, Dymov S, Szyf M and Meaney MJ. 2004. Epigenetic programming by maternal behavior. Nat Neurosci 7:847-854.

\section{GENE-ENVIRONMENT INTERACTIONS Reported by Heather Volk}

A model was proposed for trans-synaptic effects in the development of a severe form of Attention Deficit Hyperactivity Disorder (ADHD) combining known genetic and environmental risk factors. The data used were from the Missouri Twin Sample, a populationbased sample of twin youth ascertained from birth records in the state of Missouri (Neuman et al., 2005). Severe combined type ADHD (SC) was determined by probabilistic assignment using latent class analysis as described previously (Neuman et al., 2005). The authors examined risk for SC due to a VNTR in the $3^{\prime}$ region of the dopamine transporter gene (DAT1), an exon repeat in the dopamine $\mathrm{D} 4$ receptor gene (DRD4), and a SNP in the cholinergic receptor, nicotinic, alpha 4 (CHRNA4) gene. SC risk as a result of prenatal exposure to nicotine was also examined. The authors previously reported significant two-way interactions between DRD4, DAT1, and prenatal smoking (Neuman et al., 2007). Additionally a significant two-way interaction between CHRNA4 and prenatal smoking was found to increase the odds of combined subtype ADHD compared to more mild ADHD (fewer symptoms;Todd and Neuman 2007). Three way interactions with the two dopamine genes and prenatal smoking further increased the odds of combined subtype ADHD. A four-way interaction test indicated a further significant increased in the odds of combined subtype ADHD. The authors conclude a trans-synaptic effect of dopamine in combined subtype ADHD. The genes examined here produce products that are in close proximity. CHRNA4 and DAT1 are pre-synaptic while DRD4 is post-synaptic and dopamine is a known morphagen. Thus, these results suggest that dopamine release may be influenced by exposure to nicotine.

In another study, interactions were examined between genotypes of the catecholamine pathway and environmental lead and manganese exposure in a sample of 150 ADHD children (47 inattentive, 50 combined, 53 controls). Blood plasma levels of lead and manganese were determined and ADHD was assessed by a semi-structured clinical interview. In addition to a diagnosis of ADHD, each child underwent testing for cognitive control (using the stop task) and reaction time variability (using the stop signal task). Analyses were conduced using linear regression. Genotypes examined were DAT1, DRD4, the adrenergic alpha 2 receptor (ADRA2A), and dopamine beta hydroxalyse (DBH). No genetic main effects were found for ADHD or cognitive processes. Exposure levels of lead and manganese were similar to the national average. However, significant correlations between lead exposure and the inattentive and hyperactive symptoms were found; no significant correlations were found between the environmental exposures and the genes examined. There was a significant increase in lead exposure for the combined subtype of ADHD compared to inattentive ADHD and 
controls. When a composite variable of having any of the risk alleles of the four genes was created, statistically significant effects were found for this genetic composite and lead exposure for increased risk for reaction time variability scores. Significant effects were also found with number of hyperactive symptoms, DBH, and lead. No significant effects were found for inattentive symptoms. However, risk for inattentive symptoms was increased for lead exposure. Cognitive control appears to be a mediator between lead and ADHD. Lead exposure is a risk factor for inattention, significantly mediated by cognitive control, but only when a genetic risk is present. Additionally, manganese exposure is associated with hyperactivity, and mediated by cognitive control, only when genetic risk is present (Nigg et al., 2007).

In a third report, the interplay of adverse life events and familial depression liability in predicting the first onset of a major depressive episode (MDE) was examined. Subjects were assessed for psychiatric conditions at baseline and at three follow up points.:1) separation (ex: death or divorce), 2) trauma (ex: war, a physical threat, rape or natural disaster, and 3) any severe event as determined by criteria for post traumatic stress disorder (PTSD). Subjects were excluded if there was evidence of lifetime major depression before baseline examination. Family history of depression and adverse events were both significantly associated with development of depression. When stratified by familial depression liability, adverse events predicted a substantially increased incidence of MDE among respondents with familial liability, but not in those without (Zimmermann et al., in press).

When examining these risk factors additively, having both a positive family history and experiencing an adverse event increased risk for first major depression beyond either family history or adverse events alone.

The last report focused on increased risk for alcoholism by an interaction of variation in the corticotropinreleasing-hormone (hCRHR1) gene and adverse life events. Two independent cohorts were used. The first, the Mannheim Study of Risk Children, was an adolescent sample with little known exposure to alcohol. The second was a sample of alcohol dependent adults. Fourteen SNPS in the hCRHR1 gene were typed in all subjects. Two haplotypes were detected and each tagged by a single SNP. They were then examined for association with alcohol dependence phenotypes in both samples. Both haplotypes were significantly associated with lifetime prevalence of binge drinking and drunkenness in the adolescent sample. Additionally, subjects homozygous for the $\mathrm{C}$ allele were at increased risk to drink more than $\mathrm{T}$ carriers when exposed to increasing numbers of negative lifetime events. In this study, CRHR1 appears to moderate the effect of stress on heavy drinking behavior. Life events, used here as the measure of stress, only affects risk among individuals with these haplotypes (Blymeyer et al., 2007).

\section{$\underline{\text { References }}$}

Blymeyer D, Treutlein J, Esser G, Schmidt et al. 2007. Interaction between CRHR1 gene and stressful life events predicts adolescent heavy alcohol use. Biol Psychiatry [Epub ahead of print].

Nigg JT, Knottnerus GM, Martel MM, Nikolas M, et al. 2007. Low blood lead levels associated with clinically diagnosed attention deficit/hyperactivity disorder and mediated by weak cognitive control. Biol Psychaitry Sep 12 [Epub ahead of print].

Neuman RJ, Lobos E, Reich W, Henderson CA, et al. 2007. Prenatal smoking exposure and dopaminergic genotypes interact to cause a severe ADHD subtype. Biol Psychatiry 61:1320-1328.

Neuman RJ, Sitdhirasksa N, Reich W, Ji TH, et al. 2005. Estimation of DSM-IV an latent class-defined ADHD subtypes in a population-based sample of child and adolescent twins. Twin Res Hum Genet 8:392-401.

Todd RD, Neuman RJ. 2007. Gene-environment interactions in the development of combined type ADHD: Evidence for a synapse-based model. Am J Med Genet B Neuropsychiatr Genet [Epub ahead of print].

Treutlein J, Kissling C, Frank J, Wiemann S, Dong L, Depner M, Saam C, Lascorz J, Soyka M, Preuss UW, Rujescu D, Skowronek MH, Rietschel M, Spanagel R, Heinz A, Laucht M, Mann K, Schumann G. 2006. Genetic association of the human corticotropin releasing hormone receptor 1 (CRHR1) with binge drinking and alcohol intake patterns in two independent samples. Mol Psychiatry 11(6):594-602.

Zimmermann P, Brückl T, Lieb R, Ising M, Beesdo K, and Wittchen H.-U. in press. The interplay of familial depression liability and adverse life events in predicting the first onset of depression during a ten-year follow-up. Biological Psychiatry.

\section{ANIMAL MODELS Reported by Michael J. Galsworthy}

The animal models session brought together a diverse set of studies focused on mechanisms of gene action in psychiatric genetics. As with all 'animal models' of psychiatric disorders, the aim is not to generate autistic, schizophrenic, or depressed animals which suffer from the same higher-level cognitive disturbances as human sufferers. Rather, the link between a gene and a human behavioral or cognitive phenotype traverses a web of complex interactions and animal models regularly provide powerful tools to begin exploring that web in a systematic way. As the six talks in this session clearly demonstrated, animal models provide resources ranging from the development of new gene-therapy tools, to close analysis of specific genes in terms of all their consequences and interactions, to separation of self versus maternal genetic developmental effects, to nominations of new genes. Simply put, animal models allow not only gene-hunting via natural or artificial 
variation, but they also allow the added benefits of controlled experimental systems in which theories can be explicitly tested and explanatory models built stepby-step.

Three of the six talks focused on modeling key neuroscience components of schizophrenia in the mouse brain. Two talks explored the functioning of serotonin via variations in associated genes, and one talk described a project aimed at generating a toolbox of brain region-specific lightweight promoters.

The first talk introduced the Pleiades Promoter Project (www.pleiades.org), which aims to generate a public resource of 160 human DNA MiniPromoters (under $4 \mathrm{~kb}$ in size each) to allow gene expression specific to brain regions of therapeutic interest. Elizabeth Simpson from the Centre for Molecular Medicine and Therapeutics, Vancouver, Canada, explained the key purpose of the project: The once highly popular area of gene therapy seems not to have delivered on its early hype. A major problem has been the lack of specificity of attempts so far; specificity in terms of clinical relevance, of insertion site and of gene expression. The talk centered on how such issues could be addressed and outlined progress so far. Essentially, the project's structure is to go from selection of genes which show region-specific expression through stages of promoter design and promoter construction whilst testing expression in knock-in mice and tailoring the specificity to ever more differentiated regions of therapeutic interest. Some 30 regions of interest were chosen (including the blood-brain barrier), and through consultations of SAGE libraries, a list of 2780 genes was screened down to 70 that were used. The endeavour not only aims to provide the scientific community with new flexible tools, but also remains open to collaboration and nomination of genes of priority. If interested in this work, please visit the website cited above.

As mentioned above, half the talks in the session concerned the characterization of genes potentially involved in schizophrenia. Looking at the strong contributions from each of these, it is tantalizing to think how these interacting genes and proteins nominated by various methods might be slowly pulled together to explain not only key properties of the disorder, but also the mechanisms underlying the drug responses, and drug response differences across individuals. The talk by David Porteous from the University of Edinburgh (with coauthors also from Canada and Japan) demonstrated neatly how genes which have been implicated by human psychiatric research can be explored in mouse models so as to investigate neuroanatomical, behavioural and drug-response consequences within a controlled framework. The study focused on DISC 1 (Disrupted in Schizophrenia 1), a gene with one of the most replicated connections with schizophrenia in the human literature. An ENU screen for missense mutations in the mouse Disc1 identified two mutations in Exon 2. The two strains, Q31L and
L100P, both suffered from shrinkage of the brain, lower prepulse inhibition (PPI) and lower latent inhibition (LI), similar to human neurospychiatric findings. Antipsychotic and antidepressant drugs could reverse some of the symptoms, but a particularly interesting distinction was the differential response between Q31L and L100P to the drug rolipram, a phosphodiesterase-4 (PDE4) inhibitor. In fact, closer examination of the DISC1 binding partner PDE4B nominated PDE4 as a potential source of the Q31L characteristics of depression and resistance to rolipram. The study now aims to explore how missense mutations in close proximity in DISC1 provide different phenotypes by differential interaction with key interacting genes/proteins.

Similarly, the talk by Kevin Mitchell from Trinity College Dublin, explored anatomical, behavioural and pharmacological consequences of mutations in Sema6A (a transmembrane semaphorin) and two interacting proteins; PlexinA2 and A4. Semaphorin and Plexin proteins have been associated schizophrenia (and other cognitive disorders) and are believed to be important in the development of connectivity in the brain. The mutants deficient in these proteins showed a variety of axon guidance and cell migration defects, including in regions which have been implicated in schizophrenia. Behavioural tests showed hyperlocomotion and working memory deficits (spontaneous alternation in a T-maze) and cortical EEG recordings showed increased power in the alpha band. As with the findings from David Porteous' group, antipsychotic treatment reversed many symptoms and so provided an extra level of support for the relevancy of the model.

The third talk in the trio focusing on the neuroscience of schizophrenia genes in the mouse was given by Tracey Petryshen from the Broad Institute in Cambridge, USA. This group had previously discovered three QTLs associated with prepulse inhibition (PPI) in the mouse, and were now using congenic lines to reduce locus boundaries, then nominate positional candidate genes with bioinformatic resources, then finally explore those top candidates using knockout, knockdown and pharmacologic studies. All three loci were reduced to less than $3 \mathrm{Mb}$ in size before transcript and expression data and neural circuitry information implicating a PPI role were queried to nominate likely candidates within the regions. In particular, two candidates were nominated for detailed study; Protein kinase C alpha (Prkca) and Rgs9.

The talk by Jacques Mallet from CRNS, Paris, began with a riddle. Although there is much evidence for a developmental role for serotonin in the prenatal brain before it later becomes a neuromodulator/neurotransmitter, nevertheless no sites of early serotonin biosynthesis have been discovered in the mouse embryo brain. With the hypothesis that the serotonin could be of maternal origin, the group crossed mice lacking tph1, a gene responsible for the synthesis of peripheral serotonin. The very striking finding was that although 
tph1 -/- mice developed normally, the embryos of tph1 - / mothers (but not embryos of wildtype mothers and tph1 -/- fathers) showed very abnormal development. The implications of the findings are very farreaching as there have been cases where genotypes have been associated with phenotypes but the mechanisms have proven elusive. It was directly suggested in this talk that there may be cases where genotypes are correlated with phenotypes only by virtue of the genotype correlating with the mother's genotype, which in turn is the true origin of the phenotype. Should it be the case that an individual's propensity to psychiatric disorder lies in aspects of their early brain development, and this development in turn is caused at least in part directly by the mother's genotype, then this would appear to open an exciting new domain of study in developmental genetics and psychiatric genetics.

The final talk in the session differed from the others in that it used primates rather than mice as its subject of study. Ned Kalin from the University of Wisconsin explained that rhesus monkeys, like humans, have a promoter repeat polymorphism in the serotonin transporter gene. In humans, this SLC6A4 promoter repeat polymorphism has been associated with anxiety and increased amygdala response. Therefore this study examined differences between 'short' and 'long' alleles in rhesus macaque juveniles in response to differing levels of stress. These levels were; baseline in their home cages, alone in a cage, or alone and presented with the profile of a human stranger (but with no eye contact). Images from high-resolution FDG microPET following the challenges showed that although there were no differences in baseline recordings, the 'short' allele juveniles showed more activation in anxiety-relevant brain locations such as the amygdala and orbitofrontal cortex (OFC) during the stronger challenges. Thus the studies not only provided evidence for the link between the serotonin transport and anxiety in a parallel species, but also provided a nice demonstration of the elicitation of allelic differences by changing environmental circumstances.

In summary, there was both convergence and great divergence in this series of talks. The convergence in the schizophrenia genes being uncovered and investigated in the mouse brain gave promise of the sophisticated integrated models that are surely to come. The divergence in the roles of serotonin, one investigating gross brain deficits caused by lack of maternal serotonin and the other demonstrating the subtle interplay of genetic variants with very short time periods of environmental stress, remind us of the vast interactive complexity we are attempting to explore. Finally, the development of gene-therapy tools using knowledge of differential expression patterns in the brain indicates how in the future we might begin converting disease genes from objects of study to tools of medicine.

\section{FROM GENES TO DRUGS: WHAT ARE THE OBSTACLES}

\section{Reported by Petra Zimmermann, Ph.D.}

A panel discussion was led by Alan Cross and Hugh Salter from Astra Zeneca in the hope of a stimulating a dialogue about how both companies and academia could work together in academic-industry collaborations. Suggestions were solicited for optimizing future collaboration. The panel included representatives from other pharmaceutical companies, as well as academia and the federal government.

The majority of medicines today are aimed at treating the symptoms of disease rather than the underlying causes. Genetics offers the possibility to gain insight into the biological mechanisms of complex diseases such as schizophrenia. By identifying key susceptibility genes we will be able to study the encoding proteins and to discover the underlying biological mechanisms of the disease. Drugs targeting key elements in the pathway may be effective tools to disrupt pathogenic processes. However, due to fast technical developments, the availability of genome-wide studies, and the consequentially growing complexity of analysis, the translation of findings in schizophrenia genetics into effective drugs is a considerable challenge. This could make the initiation and development of collaborative strategies between academia and industry even more essential since they could provide extended power to gain more knowledge on topics as the exploration and validation of more drug targets, the identification of surrogate markers and modulators of response to a drug, and the development of a individualized and targeted medicine - to mention only a few.

Hugh Salter introduced the session with a talk on "Psychiatric genetics and drug involvement", and asked the broad question of how psychiatric genetics could be used for drug discovery. An ideal approach he said should be based on two hypotheses: First, biologicallyexplainable single or combinatorial markers predict the risk of being affected by a specific disease and second, susceptibility genes can help to identify novel routes for interventions. However, the ultimate use of genetics will take considerable time because of concerns over replication and validity, and the fact that novel targets are not processed with rapidity. Thus, there is a gap between what is ideal and the actual situation, aside from being due to the long time it will take to use genetic discoveries, the costs, the scientific rationale may be difficult to prove, many of the genes found may have small effect sizes, and disease rather than drug response is being currently assessed. It remains to be seen, if genome-wide analyses will give the answers. The question of how this gap could be bridged is closely related to the question of how industry and academia could work together. The establishment of a community for sharing results and resources is needed (e.g., sharing of DNA from pharmaceutical trials, pooling samples for 
replication, sharing data for meta-analyses, and system biology collaborations). The feasibility, desirability, and organisation of such collaborations were discussed at this session and deserve further discussion by the field in the future.

David Porteous (Chair of Human Molecular Genetics \& Medicine, Medical Genetics Section, University of Edinburgh, UK) focused on "From genes to target - how will this happen?". Can gene discovery lead to biomarker discovery and ultimately drug target development. Problems that exist with current drugs include the unresponsiveness of one third of patients, several unacceptable side effects, or weak pharmacological rationale. In psychiatric genetics, we have clear evidence that genes are important, but many aspects of the genetics are still unclear, i.e. the genetic architecture of specific diseases, the contribution of common and/or rare genetic variants, whether these diseases are dichotomous or along a spectrum, and whether unique or common underlying traits play a role. Lessons could be learned from Alzheimer's Disease where multiple genes (e.g. ApoE4, APP, PS1, PS2) are relevant. Thus, the following sequential gene based strategy was suggested: take any gene of which you have evidence for causality in an individual, family, or population, work out its biology, develop a cell based assay, identify its therapeutic effect, test the effects in animal models, conduct a trial in genetically susceptible human subjects, and test for generalisability.

As multiple independent genetic linkage and association studies provide confirmatory evidence for involvement of the disrupted in schizophrenia (DISC1) gene in schizophrenia (e.g., Blackwood et al., 2001; Harrison \& Weinberger, 2005; Millar et al., 2000), DISC1 was discussed as an example of a target for further research. DISC1 interacts with proteins required for neurodevelopment and neuronal function: The gene encoding phosphodiesterase 4B (PDE4B) is disrupted by a balanced chromosomal translocation on Chromosome 1 in a subject diagnosed with schizophrenia. PDE4B, in turn, inactivates intracellular cyclic adenosine monophosphatase (cAMP), which is a key second messenger in the brain involved in learning and memory processes as well as mood. Millar and colleagues (2005) found interactions between DISC1 and the UCR2 domain of PDE4B and they could show that increases in cellular cAMP are followed by a dissociation of PDE4B from DISC1 and an elevation in PDE4B activity (Millar et al., 2005). Clapcote and colleagues (2007) then demonstrated that two independent missense mutations in mouse DISC1 evoke distinct physiological, pharmacological, neuroanatomical, and behavioural phenotypes. Murdoch et al. (2007), furthermore, revealed that DISC1-PDE4 interaction is isoform specific. Overall, DISC1 has multiple functional motifs and interaction domains, multiple targets for mutation and modulation, and mutation class specific effects. It is a scaffold protein regulating key neurodevelopmental and neuro- signaling proteins. Therefore, there is emerging evidence that DISC1 is as a common genetic and biologically plausible risk factor for major mental illness such as schizophrenia and depression. The investigation of DISC1 mechanisms provides the opportunity for a better understanding of these complex disorders which is a prerequisite to the development of rational and effective interventions. Next steps to elucidate the DISC1 pathway should be genomic resequencing, development of more mouse models, refinement of biomarkers and endophenotypes, and drug discovery, genetic and chemical phenotypic rescue, and replication of this process. To increase the possibility of success, the specification of common goals with industry is essential.

Bryan DeChairo from Pfizer, dealt with the topic "Genetic markers for efficacy and safety in psychiatric drugs - what is the potential?". He focused on the potential of genetic markers a) to increase the efficacy and b) to enhance the safety of psychiatric drugs. While the first point aims to identify novel therapeutics targeted for patients with an unmet medical need, the second point has the goal to exclude "at risk patients" from treatment when alternative treatments exist and to proceed towards individualized, tailored medicine.

One of the problems addressed was that the predictability of biomarkers has to be extremely high. It was concluded that biomarkers should be examined over time and a combination of multiple markers could yield some advantage.

Possibilities for overcoming barriers between industry and academia that have existed in the past were discussed and commitments for future collaborations offered, despite the different ultimate purposes of commercial companies versus academia. The competition between companies and the lack of previous openness would have to be overcome. Other issues discussed were different timing of public sharing between industry and academia, the difficulties of contractual agreements with different academic versus industry criteria for intellectual property rights and other agreements. In summary, the workshop made evident that - even if various problems exist with industry-academic partnerships in psychiatric genetics, they should be encouraged in order to enhance the power to gain new insight into the underlying pathogenic mechanisms of disorders, to elucidate new ways for mechanism-based and individualized interventions, and to considerably improve the translation of these research findings into new therapeutics.

\section{$\underline{\text { References }}$}

Blackwood DHR, Fordyce A, Walker MT, St Clair DM, Porteous DJ, Muir WJ. 2001. Schizophrenia and affective disorders-cosegregation with a translocation at chromosome 1q42 that directly disrupts brainexpressed genes: clinical and P300 findings in a family. 69:428-433. 
Clapcote SJ, Lipina TV, Millar JK, Mackie S, Christie S, Ogawa F, Lerch JP, Trimble K, Uchiyama M, Sakuraba Y, Kaneda H, Shiroishi T, Houslay MD, Henkelman RM, Sled JG, Gondo Y, Porteous DJ, Roder JC. 2007. Behavioral phenotypes of Disc1 missense mutations in mice. Neuron 54:387-402.

Harrison JP Weinberger DR. 2005. Schizophrenia genes, gene expression, and neuropathology: on the matter of their convergence. Mol Psychiatry 10:40-68. Millar JK, Pickard BS, Mackie S, James R, Christie S, Buchanan SR, Malloy MP, Chubb JE, Huston E, Baillie GS, Thomson PA, Hill EV, Brandon NJ, Rain J-C, Camargo LM, Whiting PJ, Houslay MD, Blackwood
DHR, Muir WJ, Porteous DJ. 2005. DISC1 and PDE4B are interacting genetic factors in schizophrenia that regulate cAMP signaling. Science 310:1187-1191.

Millar JK, Wilson-Annan JC, Anderson S, Christie S, Taylor MS, Semple CAM, Devon RS, St Clair DM, Muir WJ, Blackwood DHR, Porteous DJ. 2000. Disruption of two novel genes by a translocation co-segregating with schizophrenia. Hum Mol Genet 9:1415-1423.

Murdoch H, Mackie S, Collins DM, Hill EV, Bolger GB, Klussmann E, Porteous DJ, Millar JK, Houslay MD. 2007. Isoform-selective susceptibility of DISC1/phosphodiesterase-4 complexes to dissociation by elevated intracellular cAMP levels. J Neurosci 27:9513-9524. 